\title{
An Exploratory Investigation of Baseball Coaches' Attitudes and Experiences With Sport Psychology
}

Jesse D. Michel

West Virginia University

Follow this and additional works at: https://researchrepository.wvu.edu/etd

\section{Recommended Citation}

Michel, Jesse D., "An Exploratory Investigation of Baseball Coaches' Attitudes and Experiences With Sport Psychology" (2013). Graduate Theses, Dissertations, and Problem Reports. 3637.

https://researchrepository.wvu.edu/etd/3637

This Dissertation is protected by copyright and/or related rights. It has been brought to you by the The Research Repository @ WVU with permission from the rights-holder(s). You are free to use this Dissertation in any way that is permitted by the copyright and related rights legislation that applies to your use. For other uses you must obtain permission from the rights-holder(s) directly, unless additional rights are indicated by a Creative Commons license in the record and/ or on the work itself. This Dissertation has been accepted for inclusion in WVU Graduate Theses, Dissertations, and Problem Reports collection by an authorized administrator of The Research Repository @ WVU.

For more information, please contact researchrepository@mail.wvu.edu. 
An Exploratory Investigation of Baseball Coaches' Attitudes and Experiences With Sport Psychology

Jesse D. Michel, M.S., M.A.

Dissertation Proposal submitted to the College of Physical Activity and Sport Sciences at West Virginia University

in partial fulfillment of the requirements for the degree of

\author{
Doctor of Philosophy \\ in \\ Sport and Exercise Psychology
}
Edward Etzel, Ed.D., Chair
Edward Jacobs, Ph.D.
Bernie Holliday, Ph.D.
Vanessa Shannon, Ph.D.
Sam Zizzi, Ed.D.

College of Physical Activity and Sport Sciences

Department of Sport and Exercise Psychology

\author{
Morgantown, West Virginia \\ August 2013
}

Keywords: Baseball, Coaches, Attitudes Toward Sport Psychology, Sport Psychology

Experiences, Sport Psychology Intentions

Copyright 2013 Jesse D. Michel 


\begin{abstract}
An Exploratory Investigation of Baseball Coaches’ Attitudes

and Experiences With Sport Psychology
\end{abstract}

Jesse D. Michel

This study examined baseball coaches' attitudes and experiences with sport psychology. The researcher used a descriptive and multivariate approach to explore baseball coaches' relationship with sport psychology professionals and sport psychology services. A modified version of the Sport Psychology Attitudes Revised Coaches - 2 (SPARC-2) (Zakrajsek, 2011) was administered to survey coaches' stigma tolerance towards sport psychology, confidence in sport psychology consultation, openness to hiring a sport psychology professional, and cultural preference towards sport psychology practitioners. Additional survey items were included to address coaches' experiences with sport psychology. All 2013 members of the American Baseball Coaches Association and all 2012-2013 National Collegiate Athletic Association Division I, II, and III head baseball coaches were asked to participate. Participants completed an online survey to investigate the above-mentioned factors and the relationships between variables such as coach's age, years coaching, education level, and primary job responsibility, with coaches' attitudes and experience with sport psychology. Descriptive analyses revealed that the majority of baseball coaches had some prior exposure to sport psychology, generally had positive attitudes towards sport psychology, and intended to use sport psychology services in the future. Logistic regression analyses revealed that stigma tolerance, confidence in sport psychology, and previous experience with sport psychology can help predict coaches' intention to use sport psychology services. Findings from this study may benefit baseball coaches, athletes, and sport psychology professionals, and provide insight into the current place of sport psychology in baseball.

Keywords: Baseball, Coaches, Attitudes Toward Sport Psychology, Sport Psychology Experiences, Sport Psychology Intentions 
AN EXPLORATORY INVESTIGATION OF BASEBALL COACHES’ ATTITUDES

\section{Acknowledgements}

I would like to acknowledge the following people, without whom the completion of this dissertation would not have been possible.

First and foremost, I would like to thank my dissertation committee chair, primary advisor, guitar dealer, and philosopher extraordinaire Dr. Edward Etzel. I could not have reached this destination without your continuous guidance, encouragement, endless patience, and positive feedback over the past four years. EE, as I have told you countless times before, I really lucked into this whole thing and thank my lucky stars that you were willing to take a chance on me. Having you as an advisor has allowed me to mature as a sport psychology professional, researcher, student, and young man, and for that, I cannot thank you enough. Since I stepped foot on campus, you have challenged me to think outside the box, been a role model and confidant, mentored me through good times and bad, and were always willing to heat up a cup of coffee and chat about whatever was on my mind; You have helped make this journey extremely enjoyable and personally rewarding, and I am so thankful to call you a friend.

I would also like to express gratitude to the four other members of my dissertation committee: Dr. Bernie Holliday, Dr. Ed Jacobs, Dr. Vanessa Shannon, and Dr. Sam Zizzi. Bern, thank you for having an integral role in this project and taking the time out of your extremely busy schedule to help mentor me over the past two years. I am so thankful we had the chance to work together, and I hope that there is a lot more work to do in the future. I am eternally grateful for your guidance and advice on all things related to baseball and life. Dr. J, thank you for showing me the difference between empathy and sympathy, and for demonstrating how I can be a better professional and human being with the skills you taught us in Allen Hall. I will never forget the fun we had in your classes and appreciate the kindness and generosity you showed me 


\section{AN EXPLORATORY INVESTIGATION OF BASEBALL COACHES' ATTITUDES}

over the years. Dr. S., thanks for keeping me motivated, making our meetings more than just "work", and for attempting to make me into some semblance of a researcher. Thank you for always being there to pick me up when I got frustrated with my research or writing. I appreciate your honesty, the way you challenged me to be better, and for always playing devil's advocate when I needed a reality check. Dr. Z, thank you for teaching me what it means to have perspective, for challenging me to become a better student, researcher, and consultant, and for showing me that it is possible to live a life of balance. You have been my employer, teacher, advisor, and mentor, and I will carry many of the lessons you taught me for years to come.

I would like to thank every coach who took the time to participate in the study. Your honest and candid responses allowed me to answer some of the questions that originally got me into this field and ultimately taught me how to garner my passion into helping coaches and athletes perform to the best of their ability. I love baseball, I love coaching, and I love helping people get better. The results from this study will allow me to open up doors that would have otherwise remained closed, and for that, I am personally and professionally grateful.

I would like to thank my friends and colleagues in Morgantown. That includes everyone in the Sport and Exercise Psychology program, to my co-workers at WELLWVU and PEIA, to all faculty and staff across campus. You helped make this experience possible and I will be forever grateful for the lifelong bonds and relationships we have developed over the past four years. From football games to High Street, the 2010 Final Four to the fun we had in class, I will look back on my time in Morgantown and smile because you made me very happy. The fun we had was a great respite from the workload we endured.

Last, but certainly not least, I would like to thank my immediate friends and family. Thank you for your unconditional love and support. Thank you for always being there to pick 


\section{AN EXPLORATORY INVESTIGATION OF BASEBALL COACHES’ ATTITUDES}

me up when I get down, reminding me what it means to be loved, and for providing me with the foundation and resources needed to complete this journey. I am so lucky to have you all in my life and am fortunate that you continue to encourage me to achieve my professional and personal goals. 


\section{Dedication}

I would like to dedicate this dissertation to my family:

My father, Randy, for teaching me that passion and a great work ethic are the keys to success. Thank you for never refusing to throw batting practice, for being the best dad a boy could have, and for providing the blueprint to being a great father, better husband, and even better human being.

My mother, Jan, for teaching me that it's okay to express my emotions, to strive for nothing less than perfection, and for showing me the strength that it takes to survive. Thank you for providing unconditional love and for caring more than humanly possible. I am eternally grateful.

My sister, Lindsey, for always knowing how to make me smile and for supporting me throughout this process. "Chapes", I'm so lucky to have you in my life and truly cherish the relationship we have developed. 


\section{Table of Contents}

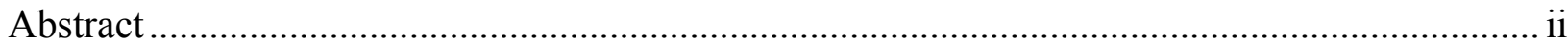

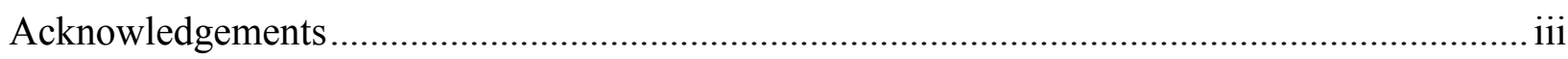

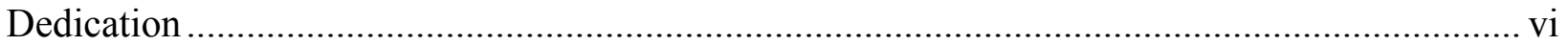

Table of Contents .......................................................................................................... vii

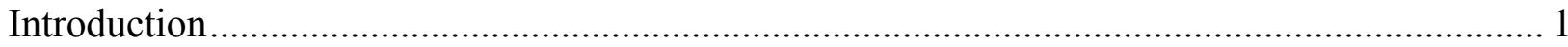

Baseball and Coaches' Impact on Athletic Development............................................... 1

Sport Psychology Professionals and Services...................................................... 8

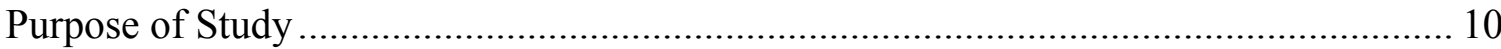

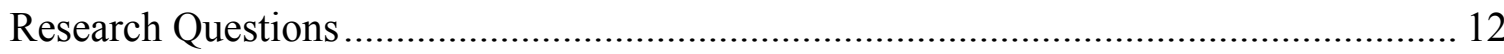

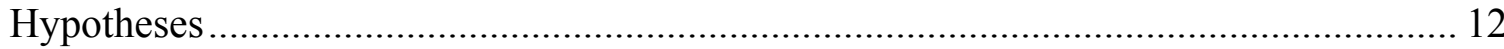

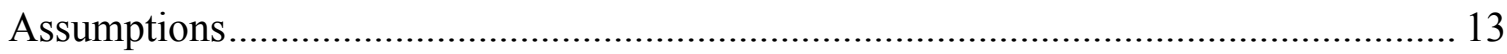

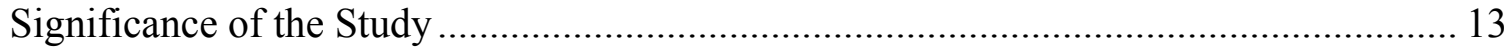

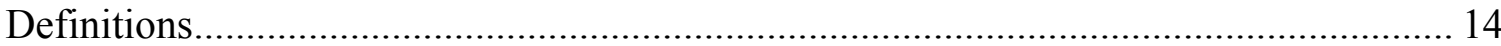

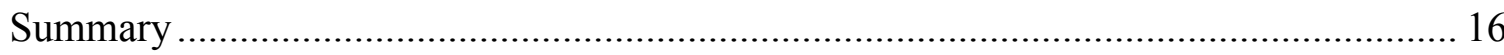

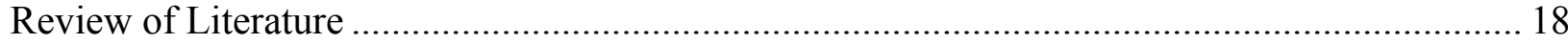

Sport Psychology and Baseball.......................................................................... 18

"The Mental Game" of Baseball....................................................................... 18

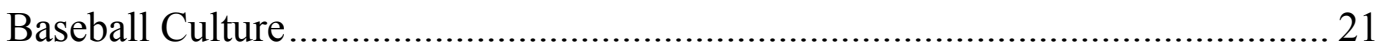

Role of Sport Psychology Professionals in Baseball ........................................ 24

The Use of Sport Psychology in Baseball: The Early Years ............................. 27

The Use of Sport Psychology in Baseball: The Modern Era ............................. 30

Attitudes Towards Psychology and Sport Psychology Services................................... 32 
Factors that Impact Help-Seeking Behaviors .............................................. 32

Attitudes Towards Sport Psychology................................................................ 35

Measuring Athletes' Attitudes Towards Sport Psychology ................................ 37

Attitudes Towards Seeking Sport Psychology Consultation Questionnaire......... 40

Sport Psychology Attitudes-Revised .......................................................... 41

Measuring Coaches Attitudes Towards Sport Psychology ................................ 42

Sport Psychology Attitudes Revised - Coaches ........................................... 46

Factors Impacting Sport Psychology Services and Professionals................................. 48

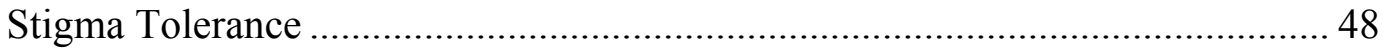

Openness towards Sport Psychology Services ............................................. 49

Perceptions of Effectiveness, Expectations, and Experiences with Sport

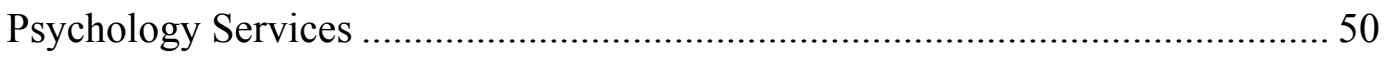

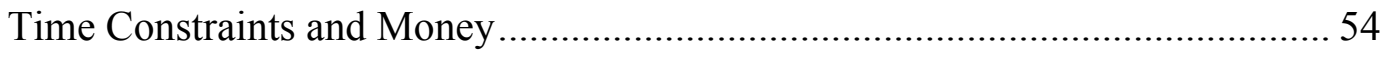

Social Influence and Gender Roles........................................................... 55

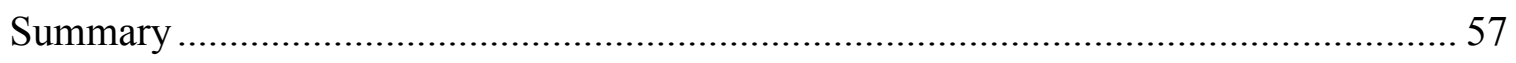

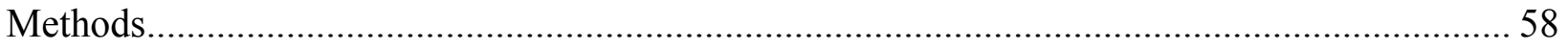

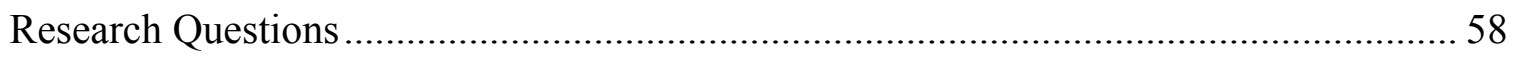

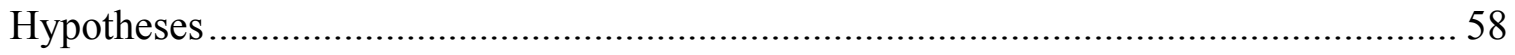

Identification and Description of Participants ..................................................... 59

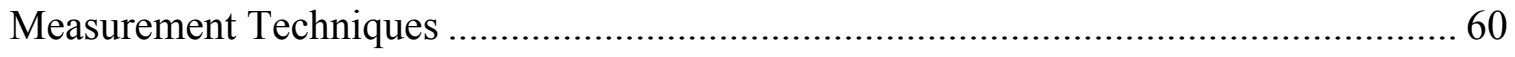

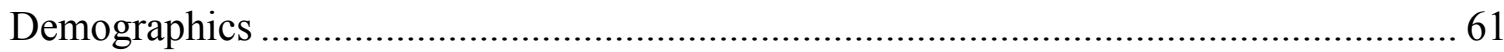

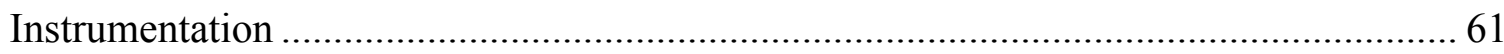

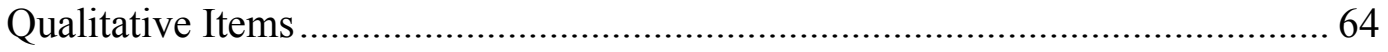




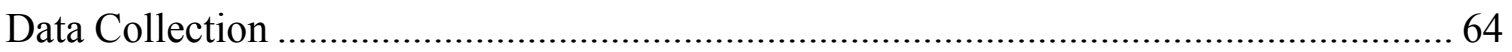

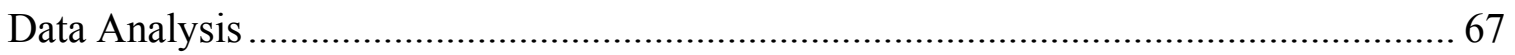

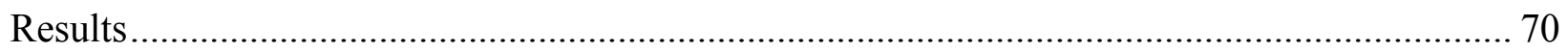

Demographic and General Sport Psychology Information............................................... 70

SPARC-2, Sport Psychology Expectations, and Subjective Norms ................................. 71

Attitudes Toward Sport Psychology Services Within Independent Variables .................. 73

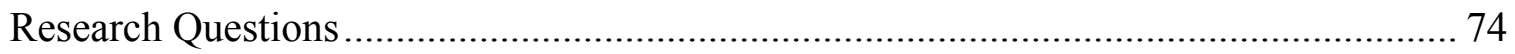

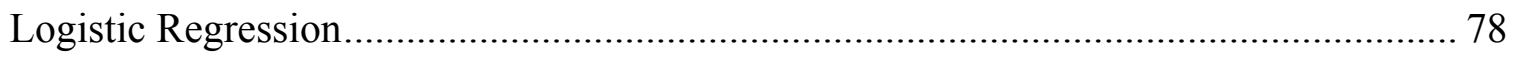

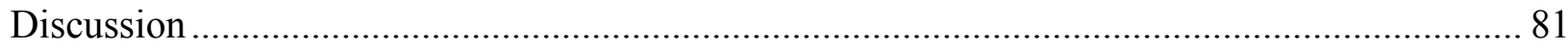

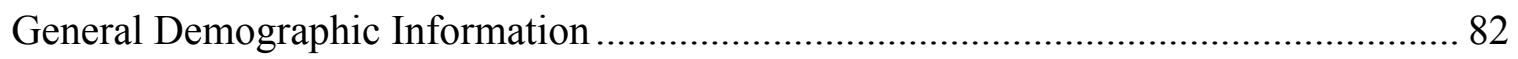

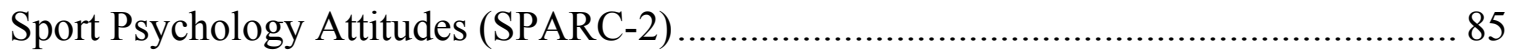

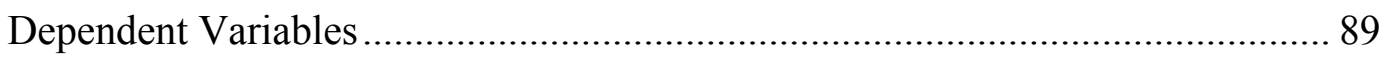

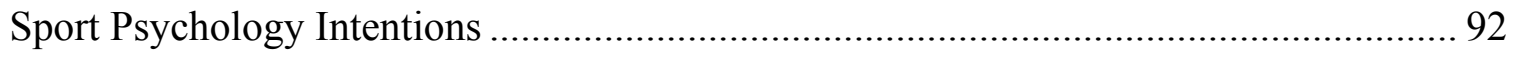

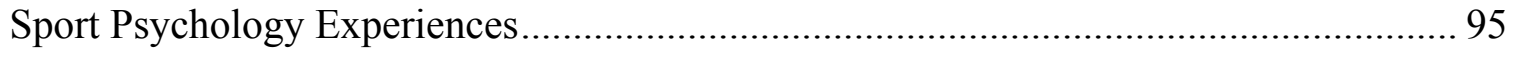

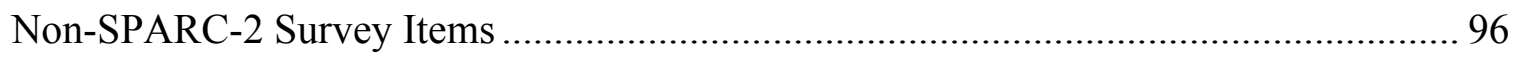

Sport Psychology Knowledge and Education....................................................... 96

Sport Psychology Expectations and Coaches Subjective Norms ........................ 101

Limitations of the Current Study ................................................................................ 103

Recommendations for Future Research ................................................................... 106

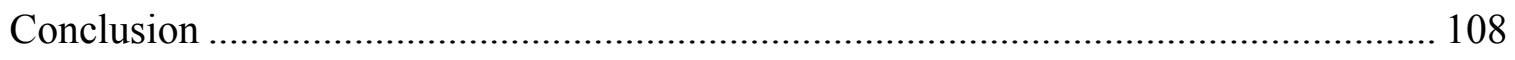

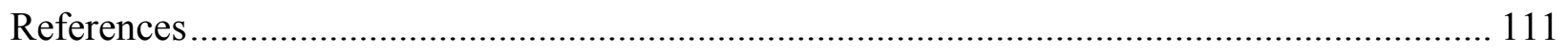

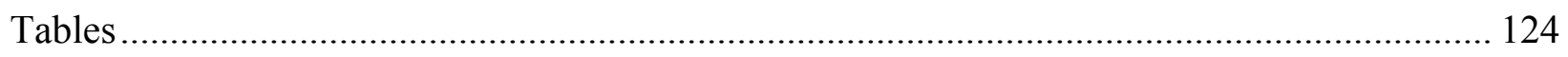

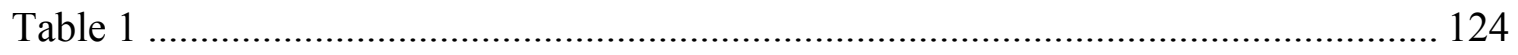


Demographic Characteristics of Participants ............................................... 124

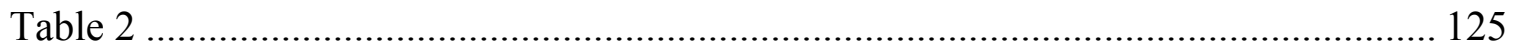

Primary Job Responsibility by “Baseball Mental Game” Demographic Variables

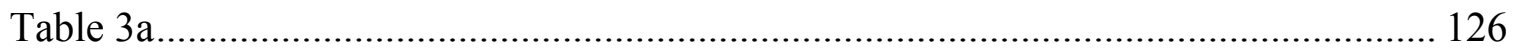

Demographic Variables and Attitudes Towards Sport Psychology.................. 126

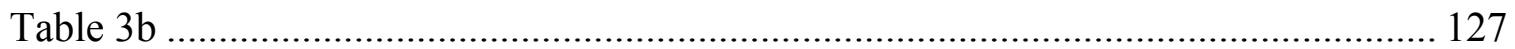

Demographic Variables and Attitudes Towards Sport Psychology................... 127

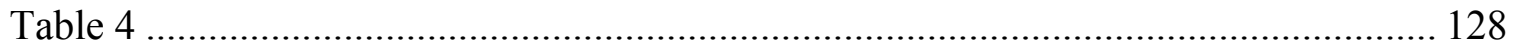

Descriptive Statistics and Correlations for Non-SPARC-2 Items ................... 128

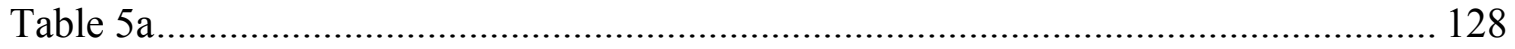

Independent Samples t-Test for "Intent to Use SP Services" .......................... 129

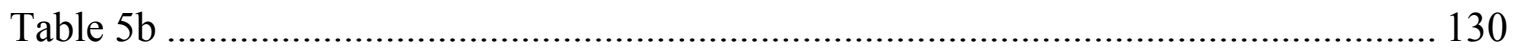

Independent Samples t-Test for "Intent to Use SP Services" .......................... 130

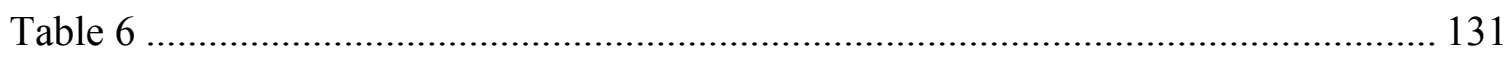

Correlations for SPA-RC Subscales ............................................................ 131

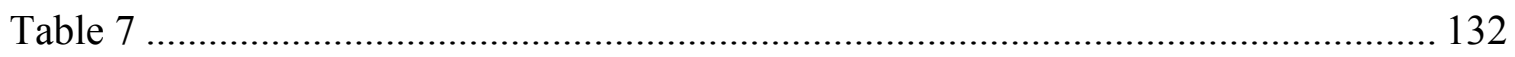

Intent to Use SP Services in the Next 12 Months ............................................ 132

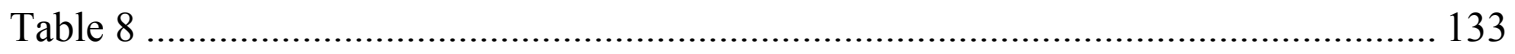

Logistic Regression and Odds Ratios For Intention to Use SP Service ............ 133

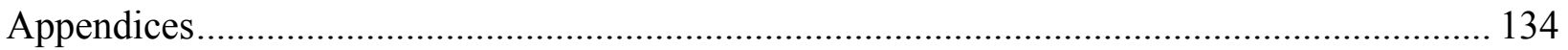

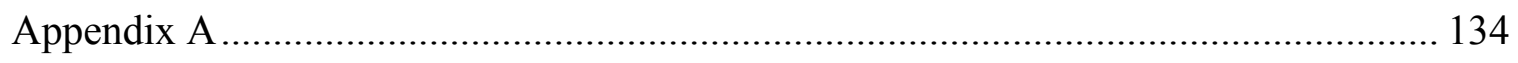

Sport Psychology Attitudes Revised Coaches-2 ....................................... 134 


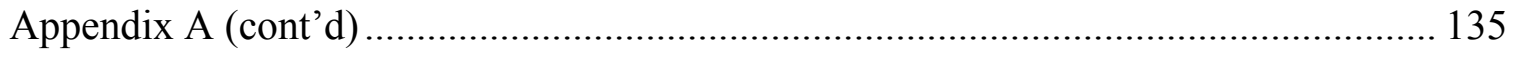

Sport Psychology Attitudes Revised Coaches-2 2................................................ 135

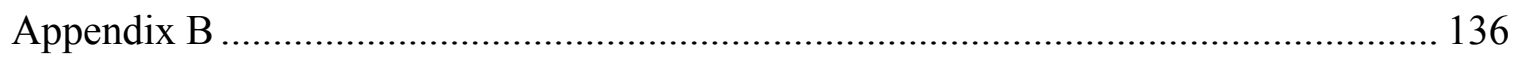

Sport Psychology Access and Experience ....................................................... 136

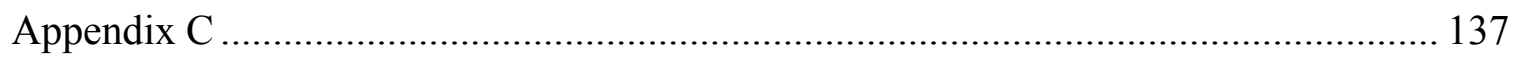

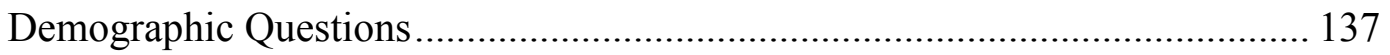

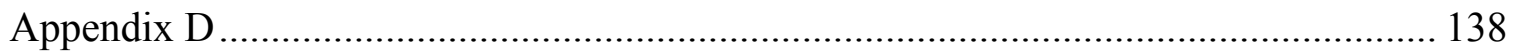

Sport Psychology Expectations and Baseball Norms Questionnaire................... 138

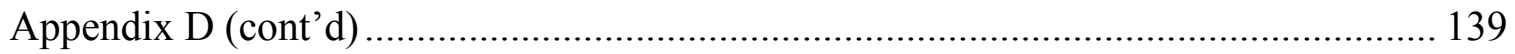

Sport Psychology Expectations and Baseball Norms Questionnaire.................. 139

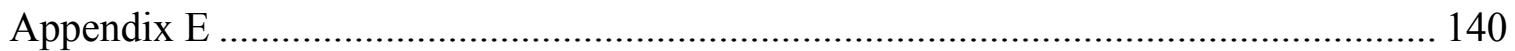

Sport Psychology Education and Baseball-Related Questions ............................ 140

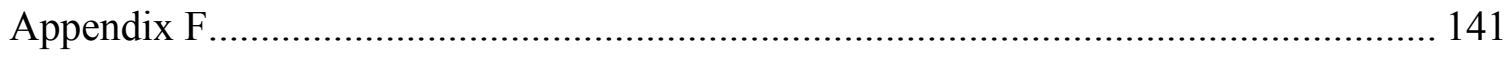

WVU Institutional Review Board Approval................................................... 141

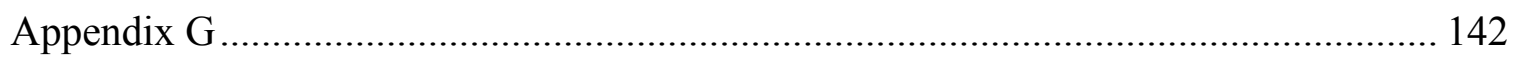

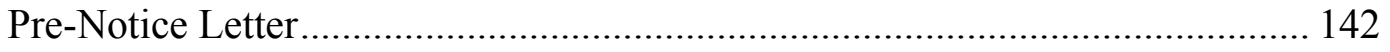

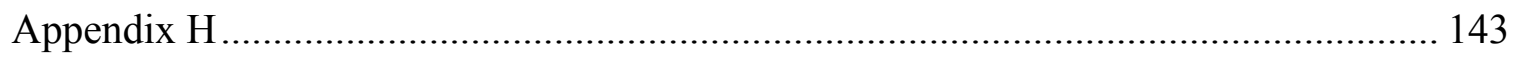

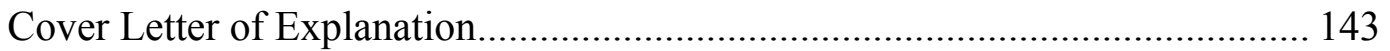

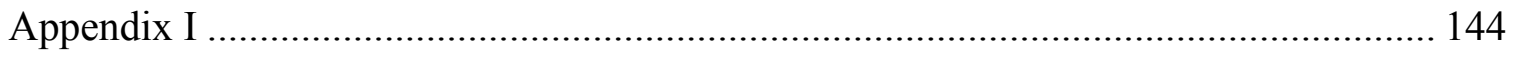

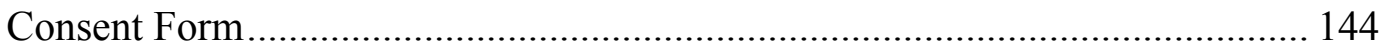



AN EXPLORATORY INVESTIGATION OF BASEBALL COACHES' ATTITUDES

\section{CHAPTER I}

\section{Introduction}

\section{Baseball and Coaches' Impact on Athletic Development}

According to a 2011 online report published by the Wall Street Journal, there are approximately 11.5 million baseball players in the United States (Futterman, 2011). An estimated two million children play Little League baseball and at least eight million play some form of youth baseball. There are approximately 455,000 high school baseball players from 15,786 schools and over 48,000 college players at 1,600 two and four-year universities across the country (Futterman, 2011). At the professional level, there are numerous independent leagues and 30 Major League Baseball organizations, each with at least five minor league affiliates. With so many baseball players taking the field every year, there are thousands of coaches charged with the task of getting the most they can out of each player.

Baseball is considered to be a game of both physical and mental skills (Ravizza, 1998), the latter of which might become even more important as players rise in age and skill level. For example, as athletes make the transition from youth to high-school competition and beyond, their physical and mental skills strength and become more fine-tuned. Coaches also spend more time with their athletes as competition level increases, giving them the chance to have a greater impact on their athletes' lives. Depending on the age group and skill level of their athletes, coaches may find mental skills have a bigger impact on performance than factors like physical development or talent level.

The type and range of teaching coaches do with their athletes may vary depending on factors such as a coach's playing experience, coaching education, and the level of competition. For example, youth (e.g., Little League, Pony Baseball) coaches are generally parents or 


\section{AN EXPLORATORY INVESTIGATION OF BASEBALL COACHES’ ATTITUDES}

volunteers, many of whom lack formal coaching education or experience. Coaches working with elementary age children must be patient as their athletes develop the basic motor skills and handeye coordination to play the game successfully. Their job is to be supportive, positive, and encourage their athletes to have fun. Once athletes reach high school, their relationship with a coach can change based on increased performance expectations and amount of time spent with the coaching staff. Schools often employ high school coaches other capacities (e.g., PE teachers) as well, allowing for more "face-time" and informal contact with their athletes. Since the difference in physiological and physical skill development for youth and high school athletes can be great, coaches at those levels may emphasize and teach the basic tactics and techniques of quality baseball, and place little to no emphasis on mental skills. In contrast, youth athletes could benefit personally and athletically from learning about the relationship between the mind, body, and performance.

At the college level, the success or failure of most coaches depends on their win-loss record. Coaching college athletes can be demanding, with coaches being responsible for furthering the academic, physical, emotional, and mental development of their athletes (Tutko \& Richards, 1971). College baseball coaches rely on 25-30 young men to put a winning team on the field each season. They dedicate a considerable amount of professional and personal time to their teams and players, and are expected to be strong recruiters, make sure their athletes maintain high academic standing, create a positive environment in their program, and most importantly, put together a successful program.

In addition to the number of practices and strength and conditioning workouts that collegiate athletes go through every season, mental skills can have a significant impact on performance. As such, college coaches would seemingly do whatever they could to gain an edge 


\section{AN EXPLORATORY INVESTIGATION OF BASEBALL COACHES’ ATTITUDES}

over the competition. Strategies might include devising smarter and more efficient tactics or strategies based on team and individual statistics, improving coaching techniques through training and education, and recognizing the importance of developing both the physical and psychological skills of their athletes.

As players talented enough to climb up the competition ladder reach the professional ranks, psychological skills may become even more important to their athletic success. The impact of mental skills on performance at the professional level can be explained in part by the fact that over half of all first round professional draft picks, arguably the most talented and scrutinized amateur players in the game, never make it to the Major Leagues (Gmelch, 2006). Considering the amount of time, money, and resources professional organizations invest in player development, it would seem reasonable such organizations would aspire to learn as much and they can about their players' physical and mental skills. Regardless of athlete age group or talent, coaches at every level have a responsibility to help their athletes work toward reaching their full potential.

The interpersonal relationship formed between a coach and athlete can be one of the most influential within sport and a critical factor in personal and psychosocial development (e.g., Aide \& Jowett, 2010). As a primary gatekeeper, teacher, and role model, coaches can play vital roles in the athletic and physical development of their athletes. Athletes may view coaches as friends, mentors, or parental figures, and often respect and trust their coach's judgments (Dieffenbach, Gould, \& Moffett, 2002). Based on the immediate respect or credibility coaches' often receive as an authority figure, and the fact that athletes may look to their coaches for guidance on and off the field, coaches may profoundly influence athletes' attitudes, perceptions, and behaviors.

Athletes' relationships with their coaches often transcend simple teaching and instruction 


\section{AN EXPLORATORY INVESTIGATION OF BASEBALL COACHES’ ATTITUDES}

(Zakrajsek \& Zizzi, 2007). For example, athletes may adopt their coaches' attitudes towards a variety of sport-specific topics such as strength and conditioning or game management, as well as topics like sport psychology. In the context of the current study, coaches' attitudes and experiences with sport psychology may play a role in athletes' perception of sport psychology and their decision to utilize sport psychology services. As such, coaches hold an essential place within athletics, and sport psychology professionals (SPPs) would be remiss to overlook how the coaches perceive sport psychology services (Zakrajsek \& Zizzi, 2007). Better understanding the factors that impact coaches' attitudes and experiences towards sport psychology may be useful information for the baseball players and coaches, as well as SPPs.

Since coaches are responsible for creating an environment where athletes can perform at a consistently high level, they could play a large role in assimilating sport psychology professionals and services into their teams. Coaches generally decide the extent to which their players have access to sport psychology information and services (Partington \& Orlick, 1987). Past attempts to explore the various sport and non-sport factors that contribute to utilization of sport psychology services focused primarily on research with athletes (e.g., Blom, Hardy, Burke, \& Joyner, 2003; Martin, 2005; Martin, Kellmann, Lavallee, \& Page, 2002; Martin, Wrisberg, Beitel, \& Lounsbury, 1997). Unfortunately, these studies did not include coaches, a component vital to the athletic development and relationship between sport participants and sport psychology. Given the increasing role coaches have on athletes' attitudes and beliefs and the continued growth of applied sport psychology, Zakrajsek and Zizzi (2007) emphasized the importance of gaining an understanding of the factors influencing coaches' use of sport psychology services.

Although sparse, there is some literature suggesting that 'decision makers' (e.g., coaches, 


\section{AN EXPLORATORY INVESTIGATION OF BASEBALL COACHES’ ATTITUDES}

owners, general managers, other management personnel, etc.) in various professional sport organizations have started to place a greater value on the impact psychological skills can have on athletic performance (Ludwig, 1996; Maher, 2011; Schinke, Hancock, Dubuc, \& Dorsch, 2006). Subsequently, it appears that some coaches are beginning to understand the positive effect sport psychology can have on their teams (Werthner \& Trudel, 2006). Specifically, research concerning elite North American sports suggests that coaches have started to seek out mental training and counseling services more for their athletes by hiring sport psychology professionals (Ludwig, 1996). For example, National Football League, National Basketball Association, and Major League Baseball teams utilize SPPs (Maher, 2011), and some National Hockey League teams have sport psychology professionals at 50-75\% of their games (Schinke et al., 2006). The specific responsibilities of these professionals may vary depending on the organization they work for, but can include evaluating future draft picks, profiling mental skills of the athletes, or doing workshops on a variety of sport psychology principles or mental skill topics such as leadership, focus, or confidence (Schinke et al., 2006).

This gradual shift in mentality is important and may demonstrate that a coach's lack of understanding or utilization of sport psychology services might simply reflect a lack of education about sport psychology. For example, some evidence suggests that coaches today are learning that players can improve their mental skills on a regular basis, not just when they need to "fix" a problem or get out of a slump (Schinke, Hancock, Dubuc, \& Dorsch, 2006). Providing more coaches with the proper knowledge, education, and experience with sport psychology could contribute to the physical and psychological well-being of both coaches and athletes. As coaches begin to further understand the role sport psychology can play in baseball, there could be more emphasis placed on both physical and mental skill development with their athletes. 


\section{AN EXPLORATORY INVESTIGATION OF BASEBALL COACHES’ ATTITUDES}

Considering that athlete performance is primarily determined by a combination of players' physical and mental skills, one would presume that baseball coaches across age groups and skill levels would appreciate and value of mental skills training. Some recent research suggests coaches may want to learn more about sport psychology and how they can apply that information with their athletes and to their coaching to help them make a more educated decision with regards to hiring a psychology professional (Zakrajsek \& Zizzi, 2007). Results from the same study suggested that coaches opinions about sport psychology and intentions to use an SPP can be impacted through education and learning about how sport psychology can impact athletes' performance. For example, after attending a workshop designed to increase perceived behavioral control, self-efficacy, openness and intentions to use an SPP, coaches became more aware of the benefits associated with utilizing an SPC and some went from having no interest to utilizing an SPC to at least thinking about what it would be like (Zakrajsek \& Zizzi, 2007). This is also encouraging because sport psychology researchers have observed that coaching effectiveness can be improved with some knowledge or exposure to sport psychology concepts (e.g., Hall \& Rogers, 1989).

Despite the apparent growing interest in sport psychology and importance baseball coaches' place on the "mental game", there does not appear to be existing data examining their relationship with sport psychology. Additionally, the attitudes towards sport psychology or the extent to which baseball coaches and teams across the country utilize sport psychology services remains unclear. On the one hand, teams and organizations understand and value the impact psychological skills have on performance, yet on the other hand, these attitudes do not necessarily reflect organizational decisions or coaches' behaviors. Take, for example, the 


\section{AN EXPLORATORY INVESTIGATION OF BASEBALL COACHES' ATTITUDES}

following quotes told to George Gmelch (2006) while interviewing a veteran professional coach for his book Inside Pitch: Life in Professional Baseball

While the importance of the mental element in performance is widely recognized, management has been slow to develop it in its players. 'It's like it doesn't matter,' said one progressive coach. 'They treat the mental side like we are all idiots. You have lots of attention going to strength and the physical tools, but the other part they don't worry about.' (p. 131)

Gmelch (2006) also discussed the fact that despite all of the research indicating performance can be improved by developing mental skills, anecdotal evidence suggests most baseball organizations and coaches pay little formal attention to these skills. He informally asked coaches from several professional teams about sport psychology and mental skills, and received an interesting mix of responses. For example, when speaking to one coach about sport psychology, he was told, "I don't do a whole lot with that. It's up to the individual. We have a sports psychologist who does that stuff in spring training. I'll leave it to him" (p. 131). When he asked another particularly eloquent 'veteran, gray-haired coach' about sport psychology, the coach hinted that he did not know anything about visualization. To be more specific, the coach responded to Gmelch's inquiry by saying "Well, I can visualize what I'd like to do with as sexy woman in an X-rated movie, but I can't see how seeing myself hit a baseball would ever be of any help" (p. 131). Gmelch (2006) focused primarily on Major League Baseball organizations, so that reaction may or not be typical, but could reveal some of the inherent stigma, biases, and complete lack of understanding and awareness towards sport psychology present among some coaching staffs. 


\section{AN EXPLORATORY INVESTIGATION OF BASEBALL COACHES’ ATTITUDES}

According to the wide variety of coaches and players Gmelch (2006) spoke with about the mental game, it appears that coaches spend relatively little time teaching mental skills of any kind. Specifically, it is estimated that as little as $10 \%$ of all instructional time in professional baseball is devoted to the mental game (Gmelch, 2006). Nevertheless, he believed that as organizations come to rely more on sport psychology professionals, evidence-based mental techniques are likely to become an integral part of pregame preparation of future baseball players. As sport psychology becomes more integrated and accepted into baseball culture, a trickle-down effect will occur from professional to collegiate sports and beyond.

While these stories, numbers, and estimates are only reflective of a select number of coaches and players, it appears that previous research on sport psychology and baseball has yielded mixed reviews; Some researchers have demonstrated that baseball coaches and organizations appreciate and value psychological skill development, while others have suggested that although coaches may value mental skills, they are not doing much to address this useful component of performance. This discrepancy, coupled with the fact that there is no research examining baseball coaches' relationship with sport psychology services or professionals, provides support to the usefulness of the current study.

\section{Sport Psychology Professionals and Services}

As the understanding of how mental skills impact athletic performance increases, coaches may seek more outside help or information about how to develop these skills with their athletes. People trained in this area of performance, sport psychology professionals (SPP), have been defined as professionals with a combination of graduate-level education and training in sport psychology, kinesiology, or sport science who are capable of helping athletes and coaches develop the psychological and emotional skills necessary for attaining peak performance and 


\section{AN EXPLORATORY INVESTIGATION OF BASEBALL COACHES’ ATTITUDES}

enhancing their quality of life (Donohue et al., 2004). There are typically two categories of services that SPPs provide: educational, and clinical (Anshel, 1990). Educational SPPs, often referred to as "sport psychology consultants" (SPC) typically have a strong background in kinesiology, physical education, or sport science. They may have some graduate training in counseling, but are not generally licensed to treat clinical disorders (Weinberg \& Gould, 2003). Educational SPPs typically teach athletes and coaches how to develop and improve mental skills for peak performance through psychological skills training (PST) (Anshel, 1990). PST can help athletes improve performance, but is not considered a "quick fix" for performance problems (Martens, 1987).

On the other hand, clinical SPPs, commonly referred to as "sport psychologists" or "sport counselors", are trained to provide athletes and coaches with clinical services that may help treat psychological problems such as depression, eating disorders, or substance abuse. To legally provide these services, practitioners must be a state licensed psychologist or counselor. The majority of their training is in psychology and/or counseling; however they are encouraged to have additional training in sport sciences to provide more effective interventions and better understand the impact sport can have on personal and athletic development.

Previous studies have shown that athletes may improve performance by learning and practicing various mental skills taught by SPPs such as positive self-talk, confidence, focus, and goal-setting. However, research indicates that when athletes do invest time and effort into improving psychological skills, they may be doing so incorrectly or inefficiently (e.g., Frey, Laguna, \& Ravizza, 2003; Monserrat, 2004). For example, in a study exploring NCAA Division I college baseball players' experiences with mental skills training, researchers found that although players utilized sport psychology skills during competition, they rarely worked on such 


\section{AN EXPLORATORY INVESTIGATION OF BASEBALL COACHES’ ATTITUDES}

skills during practice (Frey, Laguna, \& Ravizza, 2003). Although limited in scope, the previous study does conjure up an interesting dichotomy: baseball players and coaches agree that there is a strong mental component to performance, yet coaches may not know how to help develop these skills and do not set aside practice time to improve the components of the "mental game". It is curious to think that baseball players only use mental skills during competition, yet do not practice them throughout training and perhaps elsewhere during the season.

There appears to be no current clear understanding of baseball coaches' relationship with sport psychology and how that might influence their perceptions about mental skills training. What value do baseball coaches across age groups and skill levels place on sport psychology? What circumstances or barriers are prohibiting players and coaches from being exposed to and developing these skills during practice, in between games, or in the off-season? Exploring baseball coaches' views about sport psychology could help SPPs clarify the extent to which they understand or value sport psychology, the number of coaches who utilize sport psychology services, and identify potential barriers that might inhibit coaches from working with SPPs to improve athletic performance. Additionally, this information could give baseball coaches a better sense of how other coach's view sport psychology, which might lead to greater receptivity of sport psychology acceptance across skill levels. A better understanding of sport psychology could ultimately help baseball coaches improve their athletes' performance through teaching and implementation of mental skills during practice.

\section{Purpose of Study}

Over the past 40 years, the psychological aspects of performance have become a more consistent topic of conversation among athletes, coaches, fans, analysts, and media members (Williams \& Straub, 2006). Previous researchers have demonstrated the correlation between 


\section{AN EXPLORATORY INVESTIGATION OF BASEBALL COACHES’ ATTITUDES}

utilization of sport psychology and improved performance (e.g., Thelwell, Greenless, \& Weston, 2005; Weinberg \& Gould, 2011). There is some evidence to suggest there has also been an increased implementation of sport psychology principles and utilization of sport psychology professionals with amateur, international, and professional athletes over that time period (e.g., Gould, Dieffenbach, \& Moffett, 2002; Haberl \& Peterson, 2006; Ludwig 1996; Sullivan \& Hodge, 1991; Zakrajsek \& Zizzi, 2007).

Since there are significantly more athletes than coaches in baseball today, athletes are the primary consumers of sport psychology services. However, coaches are often responsible for hiring SPPs to work with their athletes and can play a direct role in an athlete's decision to practice sport psychology skills or seek help from an SPP. Some research also suggests that Olympic, college, and professional level coaches' perceptions of sport psychology can be influenced by the stigma associated with seeking help from an SPP. For example, athletes or coaches who work with an SPP can be labeled "mental patients" or "head cases" (Ravizza, 1988). Additionally, evidence suggests athletes and coaches may avoid working with an SPP due to their concern with lack of time, money, and additional resources (Ferraro \& Rush, 2000; Gould, Medbery, Damarjian, \& Lauer, 1999; Pain \& Hardwood, 2004; Voight \& Callaghan, 2001).

Considering the paucity of research on baseball coaches and sport psychology, and the potential performance benefits baseball players and teams may receive from utilizing mental training, it would seem useful to investigate baseball coaches' attitudes and experiences with sport psychology. The current study appeared to be the first attempt at exploring baseball coaches' attitudes and experiences with sport psychology. By examining their attitudes and experiences with sport psychology, both SPPs and coaches can be more informed on the current 


\section{AN EXPLORATORY INVESTIGATION OF BASEBALL COACHES’ ATTITUDES}

relationship between baseball and sport psychology. Examining these constructs will provide SPPs with insight into the current climate between sport psychology and baseball coaches across ages and skill levels. This information will help guide SPPs' approach to working with this population, and in turn, provide better services to baseball players and coaches across ages and skill levels.

\section{Research Questions}

The four research questions considered in this study were:

1. How did factors such as coach's age, years of coaching, education level, skill level coached, access to sport psychology services, or previous experience with sport psychology relate to a baseball coach's self-reported use of sport psychology principles and services?

2. How did a coach's reported level of confidence, openness, stigma tolerance, and cultural preference relate to their self-reported intentions to use of sport psychology services?

3. How did a coach's skill level coached relate to their reported stigma tolerance and openness to using sport psychology services?

4. How did a coach's previous experience with sport psychology services relate to their stigma tolerance, confidence in SP, personal openness, and cultural preference?

\section{Hypotheses}

The four hypotheses for this study were:

H1: No significant relationships would be observed between factors such as coach's age, years of coaching, education level, skill level coached, access to sport psychology services, or previous experience with sport psychology and use of sport psychology principles and services. 


\section{AN EXPLORATORY INVESTIGATION OF BASEBALL COACHES’ ATTITUDES}

H2: No significant relationships would be observed between a coach's reported level of confidence, openness, stigma tolerance, and cultural preference and a coach's self-reported use of sport psychology principles and services.

H3: No significant differences would be observed between skill level coached and stigma tolerance and openness to using sport psychology services.

H4: No significant differences would be observed between a coach's previous experience with sport psychology (i.e., as an athlete or coach) and a coach's stigma tolerance, confidence in SP, personal openness, and cultural preference.

\section{Assumptions}

1. Participants followed all instructions and procedures correctly.

2. Each participant answered each item honestly and to the best of his or her knowledge and ability.

3. The SPA-RC is a valid and reliable instrument and measures what it purports to measure (i.e., coaches' stigma tolerance, confidence in SP consultation, openness to hiring an SPP, and cultural preference towards sport psychology professionals).

\section{Significance of the Study}

There are currently more than 11 million baseball players of every age group and skill in the United States. Baseball is a game that requires both physical and mental skills, and as players get older and competition improves, coaches and players tend to agree that the "mental game" of baseball can become more important than physical skills alone. Athletes often look to their coaches to be role models and leaders, often adopting similar attitudes and behaviors that they see and hear during practice, meetings, and competition. Regardless of what age group or skill level they work with, coaches are responsible for teaching athletes how to improve the 


\section{AN EXPLORATORY INVESTIGATION OF BASEBALL COACHES’ ATTITUDES}

physical skills and techniques for successful and consistent performance. They may also play a role in helping to develop the social and psychological skills necessary for athletic achievement. As such, baseball coaches would seem to play a large role in the physical, emotional, and psychological development of these athletes.

Even though coaches' attitudes can impact their athletes and they play large role in their athletes ' psychosocial and athletic development, the there does not appear to be any research on United States baseball coaches relationship with sport psychology (i.e., coaches' attitudes and experiences with sport psychology). This discrepancy is interesting considering the role mental skills can play in performance and the importance coaches' may place on the mental game. Therefore, this study was designed to allow baseball coaches an opportunity to provide information on these constructs pertaining to their relationship with sport psychology.

\section{Definitions}

The following terms are relevant to the purpose of the study, the research questions, and provide deeper meaning behind the common words and phrases throughout the document. Unless otherwise cited, these definitions have been adapted to fit within the context of this study (English Dictionary, 2012).

Attitude: A combination of the manner, disposition, feeling, perceptions, and beliefs coaches' posses towards sport psychology.

Baseball Coach: A person responsible for teaching athletes the proper fundamentals, tactics, and techniques to be a successful baseball player. This the general term used for head coaches, assistant coaches, pitching or hitting coaches, private instructors, graduate assistants, and anyone else involved in the physical and skill development of baseball players of any age group or skill level. 
Culture: The collection of beliefs, expectations, and social norms that govern the behaviors of sport based on the attitudes and experiences of the individuals in that population.

Experience: The process or fact of personally observing, encountering, or undergoing sport psychology services working with a sport psychology professional as a coach, athlete, or in some other role connected to athletics.

Implementation: The act or instance of integrating sport psychology skills, principles, or ideas through specific drills, techniques, videos, workshops, books, or other forms of application.

Knowledge: The basic understanding of sport psychology principles and techniques, how they apply to sport performance, and how they could be valuable to coaches and athletes.

Relationship with Sport Psychology: For the purpose of this study, this refers to the collective attitudes and experiences with sport psychology.

Skill Level Coached: The developmental level (i.e., youth, high school, college, professional) a participant is working in at the time s/he filled out the survey.

Sport Psychology: The scientific study of people in sport and the application of the knowledge gained. Specifically, understanding the psychological factors that affect physical performance and determining how one's participation in sport impacts their development and overall well-being (Weinberg \& Gould, 2011).

Sport Psychology Consultant: For the purposes of this study, this term refers specifically to professionals with the education and training to help athletes develop the psychological and emotional skills necessary for attaining peak performance and enhancing their quality of life, but are not licensed therapists, counselors, or psychologists.

Sport Psychology Professional: Professionals with the education and training to help athletes develop the psychological and emotional skills necessary for attaining peak performance 


\section{AN EXPLORATORY INVESTIGATION OF BASEBALL COACHES’ ATTITUDES}

and enhancing their quality of life (Donohue et al., 2004). These may include sport psychology consultants, sport psychologists, sport counselors, or any other professional with the training and background previously specified.

Sport Psychology Principles: The collection of psychological constructs that make up "the mental game" including, but not limited to confidence, focus, emotion regulation, communication, leadership, concentration, or self-awareness.

Sport Psychology Technique: Any intervention that focuses on improving athletes' psychological skills for enhanced performance including, but not limited to visualization, selftalk, or progressive muscle relaxation.

\section{Summary}

Baseball is a game of both physical and mental skill. Baseball coaches at all levels play an integral role in the game. A portion of their job, regardless of competition level, is to further the athletic development of their athletes, and perhaps impact the personal and emotional development of their players. Coaches often act as role models and teachers; athletes often look to them for guidance, advice, and model behavior. Since athletes often respect their coaches, they may begin to adopt their coach's attitudes, beliefs, and perceptions about a wide array of subjects, including sport psychology. The current study examined baseball coaches' attitudes and experiences with sport psychology and the psychological aspects of performance. Gaining a clearer understanding of what baseball coaches' today think about sport psychology and learning about their experiences with sport psychology services can provide SPPs with useful information to help athletes and coaches address the psychological components of performance. If both groups understand the value of sport psychology, professionals may be able to help innumerable more sport participants via sport psychology services. This information could also help SPPs 


\section{AN EXPLORATORY INVESTIGATION OF BASEBALL COACHES’ ATTITUDES}

determine the most effective ways to educate coaches on the content, benefits, and application of mental training so they have a better understanding of how this information could help their athletes and teams. 
AN EXPLORATORY INVESTIGATION OF BASEBALL COACHES’ ATTITUDES

\section{CHAPTER II}

\section{Review of Literature}

This literature review is separated into five separate parts detailing past research about (a) sport psychology and baseball, (b) theoretical understanding of intentions and behavior, (c) attitudes towards psychology and sport psychology services, (d) factors impacting sport psychology services and professionals, and (e) a summary.

\section{Sport Psychology and Baseball}

\section{"The Mental Game" of Baseball}

Coaches generally agree that the mental components of sports can play a critical role in the performance of athletes and teams (Janssen, 1994). Researchers have demonstrated that coaches believe developing the mental skills of their players can help improve how their athletes and teams execute on the field, in the pool, and on the court (e.g., Ravizza, 1998). Baseball coaches and athletes have identified psychological skills such as pregame preparation, visualization, focus, concentration, "locking in" to every at-bat and pitch, staying "even-keel" throughout the season, and learning to deal with failure as particularly important to baseball performance (Gmelch, 2006). Similar to other sports, the "mental game" of baseball is often considered a vital element in determining which players and teams perform to their potential, especially at the collegiate and professional levels (Gmelch, 2000). For example, after working with college and professional baseball players for over fifteen years, Hanson and Ravizza (2003) stated that players typically report only $20 \%$ of their performance is determined by physical factors, and $80 \%$ or more of their performance is based on emotional and mental factors alone. Similarly, anecdotal interviews with elite level baseball coaches and players put the number somewhere between $80-90 \%$ at any given time (Gmelch, 2006). 


\section{AN EXPLORATORY INVESTIGATION OF BASEBALL COACHES’ ATTITUDES}

Kornspan and MacCracken (2003) noted that over the past twenty-five years, coaches, upper management, and other decision-makers in professional baseball have started to focus more on the psychological aspects of the game. Over that time, several Major League Baseball (MLB) organizations have gone as far as hiring sport psychology consultants (SPCs) to teach players throughout their organization skills that will help them gain more control over their thoughts, emotions, and performance (Maher, 2011). Various organizations have also started assessing players' mental abilities through psychological tests and other objective measures (Gmelch, 2000). With so much money invested in talent and development, some of these multimillion dollar "companies" have found value in developing the psychological skills of their athletes.

Despite the important emphasis coaches and players put on their mental game, they often focus their efforts less on the mental processes than the physical components (Hanson \& Ravizza, 1998). Hanson and Ravizza (2003) also emphasized that most young professional (i.e., age 18-25) ballplayers need help being convinced that their mind plays a critical role in their performance. Since baseball players can be drafted from high school, early professionals are generally the same age as college players. It is curious that baseball players of this caliber would need to be convinced that their mental skills could heavily impact their play on the field. It is possible that players inherently know their mental skills play a role in performance, but do not know how to verbalize their thoughts, seek out sport psychology services, or are unaware that there is an entire field of study dedicated to helping athletes improve performance through mental skills training. On the other hand, the specific culture of baseball could prohibit players and coaches from openly discussing the psychological impact of performance, leaving it up to 


\section{AN EXPLORATORY INVESTIGATION OF BASEBALL COACHES’ ATTITUDES}

the athlete to learn and develop those skills without any outside help or search for additional resources on their own accord.

There are several ways baseball players can learn about sport psychology and mental skills training. Players have access to hundreds of books on the "mental game" of baseball and sport psychology; there are free websites where players can learn about how the relationship between the mind and body, and there are thousands of popular media articles with coaches and player quotes about the value they place on mental toughness.

In addition to popular books, videos, or media articles, coaches could also play a critical role in communicating the value and essence of sport psychology to their athletes. One would be hard-pressed to find a coach who does not acknowledge the mental component to athletics. If coaches believe in the importance of the mental game are not educated or trained in sport psychology, they would ideally hire people who are. Subsequently, one would think coaches would be motivated to either a) learn about sport psychology and better understand how to apply sport psychology principles, or b) hire SPPs to teach their athletes and coaches how to develop mental skills. On the contrary, more often than not, sport psychology consultants seem to be on the outside looking in, knocking voraciously to get coaches to crack open the door and invite them to the party.

Contrast sport psychology with another value added service to baseball teams: strength and conditioning. High school and college athletic departments across the country employ thousands of strength and conditioning coaches because coaches and administrators understand the relationship between increased strength, agility, speed, and improved performance. Since coaches are not experts or trained in strength training, coaches invest their resources (i.e., time and money) in people who are, and hire professionals with the education and training to help 


\section{AN EXPLORATORY INVESTIGATION OF BASEBALL COACHES’ ATTITUDES}

athletes increase their speed, agility, and power. However, unlike strength and conditioning professionals, who are widely accepted and expected component of many coaching staffs, sport psychology professionals do not appear to be a common component of player development teams. What is stopping baseball coaches from hiring sport psychology professionals? Is it time, money, or lack of additional resources? How many baseball coaches have sport psychology professionals on staff or use sport psychology services? Do they feel they can teach these skills themselves? The alternative explanation would be that coaches have no interest in investing their time, money, and energy in improving their athletes "mental game", something that would be useful for SPPs to know. Whatever the case may be, and despite the importance that baseball players and coaches put on the mental side of the game, there is a paucity of research looking at the relationship between sport psychology and baseball. Specifically, researchers have yet to examine baseball coaches' attitudes, beliefs, and previous experiences with sport psychology.

\section{Baseball Culture}

Every sport has a unique "culture", or set of written and unwritten rules that govern the attitudes, behaviors, and perceptions of the athletes and coaches in that particular sport. The culture of a sport may include specific terminology or phrases used during practice or competition (e.g., Baseball players yell, "hum now kid"; Swimmers yell "hup, hup, hup") an understood hierarchy among athletes and coaches (e.g., Assistant coaches may be responsible for picking up trash after games), or unwritten rules that govern the way players dress for competition (e.g., basketball shorts worn below the knees). Since the culture of each sport is different, SPPs must first identify the culture, and then figure out how they best fit into that culture in order to be effective. Conversely, it is important that athletes and coaches feel SPPs 


\section{AN EXPLORATORY INVESTIGATION OF BASEBALL COACHES’ ATTITUDES}

understand and fit within their sport and culture (Gould, Medbery, Damarjian, \& Lauer, 1999; Pain \& Harwood, 2004). Part of knowing the culture is figuring out where it comes from and becoming knowledgeable about the intricacies of the game. Sport psychology professionals who work with baseball players and coaches must learn to know and appreciate the unique culture to be successful (Ravizza, 1988). If an SPP has no background in the sport, it is his or her responsibility to learn as much about the sport as possible before working with athletes or coaches (Ravizza, 1988).

Like all sports, baseball has a unique culture, which includes a set of written and unwritten rules and social norms that govern the sport at every level. Accepting, understanding, and abiding by the rules of this culture can be critical for SPCs to be effective. The baseball culture can be ruthless, and has been known to "eat up" consultants who are inauthentic and phony (Hanson \& Ravizza, 2003). Ravizza (1988) identified lack of sport-specific knowledge and inadequate knowledge of and experience with the politics of the sport environment as factors that impact may play a role in a sport's culture and impact SPP effectiveness. For sport psychology professionals working in baseball, the old adage "fake it "til you make it" does not seem to apply (Hanson \& Ravizza, 2003).

As cultural anthropologist, college professor, former professional baseball player, and peer-reviewed author on the subject George Gmelch outlined in his book Inside Pitch: Life in Professional Baseball (2006), the socially acceptable behaviors and norms that make up the culture of baseball can be split into two broad categories: performance and professionalism. The former reflect behaviors that may negatively affect job performance such as curfews or pre-game activities such as stretching or batting practice. The latter includes the socially acceptable rules or behaviors that reflect the 'right way' to play the game, such as a dress code, superstitious 


\section{AN EXPLORATORY INVESTIGATION OF BASEBALL COACHES’ ATTITUDES}

behavior, or rules against cursing. Perhaps more important than the rules themselves, is the notion that "players learn that some level of conformity to the norms of the team is required if they are to fit in and be accepted" (p. 51). Gmelch continues:

Behaviors that are reinforced and rewarded by coaches and teammates tend to be repeated, while behaviors that provoke comment or criticism tend to be avoided. The patterned regularities that result from this kind of learning usually remain implicit, that is, they rarely rise to the level of conscious awareness. Indeed, apart from baseball strategy, players have difficulty articulating what they have learned, or what the norms of pro [or any other level of] baseball are. (p. 51)

Previous research on baseball has resulted in the identification of several additional factors that contribute to the unique culture of the sport. Gmelch (2006) discussed specific baseball related concepts like showing respect for the game (e.g., knowing something about baseball history), putting the team before oneself (e.g., giving credit to teammates in post-game interviews), acquiring a nickname, and learning proper baseball lingo (e.g., words such as “dinger", "tater", "frozen rope"). Hanson and Ravizza (2003) identified the following areas that contribute to the distinct culture of baseball: (a) old school vs. new school approaches to game strategy (b) masculinity/gender roles, (c) over-thinking, (d) superstition, (e) politics, and (f) prospects. Without proper knowledge of the sport, clear understanding of these terms in the context of baseball, and experience working with baseball players and coaches, it may be difficult for an SPP to find work in the game. Similar to other sports, it is evident that baseball has a unique culture encompassed by a wide array of written and unwritten rules, subjective norms, and general assumptions that players and coaches consciously and subconsciously abide by. 
AN EXPLORATORY INVESTIGATION OF BASEBALL COACHES’ ATTITUDES

Previous research has yet to determine how baseball coaches and players perceive the field of sport psychology and sport psychology professionals, and has yet to examine if and how SPPs fit within baseball culture. Based on pervasive societal attitudes towards help-seeking behaviors and Gmelch's (2006) assessment of baseball culture, it appears that 'automatic' thinking patterns, lack of self-awareness or reflection, and tendency to conform to what is socially desirable and acceptable by one's team and the coaching would staff likely impact coaches' and athletes' attitudes towards sport psychology services and professionals.

\section{Role of Sport Psychology Professionals in Baseball}

By and large, baseball coaches are trained to teach their athletes the technical and strategic aspects of the game that can lead to success such as hitting, fielding, and pitching. However, research has yet to examine what coaches are doing to address mental skills like focus, confidence, or controlling emotions under pressure. In some cases, athletes can develop the skills without additional help, and in others, they fail to develop these skills altogether, leaving both coaches and athletes searching for answers as to why players and teams are not performing to their potential. Without the help of an SPP or coach who understands the role sport psychology plays in performance, mental skills can be left for athletes to develop on their own. Sport psychology consultants could fill the gap and help athletes develop the mental skills necessary for improved performance.

Sport psychology professionals are generally trained to help players develop the mental components necessary for improved performance. Sport psychology professionals working in baseball are tasked with "educating players and coaches on the fundamentals of the mental game and provide a structure of support for them as they develop their abilities to use their thoughts and emotions to the best of their advantage" (p.191) (Hanson \& Ravizza, 2003). In other words, 


\section{AN EXPLORATORY INVESTIGATION OF BASEBALL COACHES’ ATTITUDES}

SPPs train coaches and players on how knowledge of the mental game and its impact on performance can be extremely helpful as athletes' physical abilities progress with age.

Hanson and Ravizza (2003) contend the rules of the game are set up to make things difficult and force players to deal with failure. For example, components such as hitting a round ball with a round bat, having nine players on defense compared with one on offense, and the fact that results of the game are often out of any one player's control create an environment where failure is more likely than success. It is an individual sport played in a team context, and the amount of "down-time" between pitches can often be more of a mental challenge than a physical one. At its core, baseball is a game of constant adjustments and the nature of the game poses unique challenges that could make sport psychology professionals a useful resource for coaches and players.

The goals of SPCs in baseball should be to help the athlete develop the tools and mind-set needed to let go and get out of his own way, and free himself from external obstacles (Hanson \& Ravizza, 1998). Hanson and Ravizza (1998) contended that maintaining confidence and positive reactions to adversity are desired goals of psychological improvement. Ultimately, athletic performance can be enhanced by positive and confident mental attitudes. Ravizza (1990) suggested that SPCs in baseball provide information, teach mental skills, and support players and coaches in refining and developing their mental approach to the game. The three general objectives include a) long term excellent performance, b) self-adjustment, and c) consistency, or making bad days less bad and less frequent (Ravizza, 1990). An SPP that has the ability to gain the athlete's trust can be an extremely valuable resource for baseball teams because they can help players learn how to focus on factors they can control, instead of wasting mental energy on things outside their sphere of influence. 


\section{AN EXPLORATORY INVESTIGATION OF BASEBALL COACHES’ ATTITUDES}

For sport psychology professionals working with baseball players, especially at the elite levels, it is vital to get support from the coaches at the top of the organizational chain. At the high school and collegiate level, the top of the organizational chain is the head coach, otherwise known as the "gatekeepers" of amateur baseball. Coaches can be in charge of hiring assistant coaches, recruiting new players, and fundraising for their program. High school and college coaches fill a number of roles, including CEO, GM, and CFO. They also determine who is allowed into the inner circle of their "program" and can create an environment where SPPs are valued and accepted. At the professional level, owners and general managers comprise the top of the organizational chain, yet coaches still may be involved in the decision to hire sport psychology professionals (Gmelch, 2006).

If the top coaches in the organization support mental training programs, there will be a trickle-down effect throughout all levels (Hanson \& Ravizza, 2003). Similar to uppermanagement support at the professional level, support from NCAA, high school, and youth league coaches will result in players benefiting from the services provided by the SPP; conversely, weak support from the coaching staff will make it tougher to have an impact on the players (Hanson \& Ravizza, 2003). Based on previous research, one would presume that an examination of baseball coaches would result in similar findings, emphasizing the importance of the SPP developing a relationship with the coach and in turn, gaining support from the players. However, there has been a dearth of research examining baseball coaches' attitudes and experiences with sport psychology services.

Based on publicly available information and personal communication with sport psychology professionals, there seems to be an increasing number of SPPs working with Major League teams over the past several years. It appears this trend has resulted in a trickle-down 


\section{AN EXPLORATORY INVESTIGATION OF BASEBALL COACHES’ ATTITUDES}

effect as well; college baseball coaches at various universities have hired SPPs to help teach their athletes the mental skills important for athletes' success. For example, former Long Beach State Head Baseball Coach and 1989 NCAA Coach of the Year Dave Snow said, "Incorporating the mental game into [his] coaching has provided [him] with a vocabulary to address one of the most important aspects of baseball” (p.191) (Hanson \& Ravizza, 2003). Additionally, coaches could be more attentive to the potential benefits of mental training and its impact on performance based on the increased media attention given the field of sport psychology. Despite these trends, relatively little research has examined the specific relationship between baseball coaches' attitudes and experiences with sport psychology services.

\section{The Use of Sport Psychology in Baseball: The Early Years}

It has been almost 75 years since Coleman Griffith, widely acknowledged as the "Father of Sport Psychology", combined his psychological background and interest in athletics to make several important discoveries regarding baseball and psychology (LeUnes, 2000). Griffith had a sharp baseball mind, a heightened awareness of athletes, and a deep understanding of the physical and mental aspects baseball players face on a daily basis. In the late 1930s, the Chicago Cubs gave Griffith, a professor of kinesiology at the University of Illinois, the opportunity to work with their ballclub. Through his research, Griffith found that by having unproductive practices, seldom challenging players, and rarely demanding intensity and focus, players and coaches from the Chicago Cubs and other Major League Baseball teams were not reaching their full potential. He also came to the conclusion that managers needed to learn how to motivate players on an individual basis, rather than using one strategy to motivate the entire team (Kornspan \& MacCracken, 2003). 


\section{AN EXPLORATORY INVESTIGATION OF BASEBALL COACHES’ ATTITUDES}

In his pioneering work with baseball teams, Griffith discovered several ways to increase players' focus and concentration and improve practice methods. For example, he recommended that coaches be responsible for teaching players only one specific component of the game (e.g., hitting coach, pitching coach), a commonly used practice in today's game. He also understood the importance of efficient practice plans, valued pre-game routines, and understood the significance of practicing in game-like conditions. It has been almost 75 years since Griffith first challenged the status quo and forced baseball players and coaches to approach the game with a different perspective; he stressed the important aspects of baseball and understood how the mental and physical aspects of baseball were essential to performing successfully and consistently. The ideas Griffith had in 1938 were far ahead of his time. His findings, coupled with evidence-based research demonstrating the benefits of mental training formed the groundwork for the implementation of sport psychology principles and utilization of services with baseball players and coaches (LeUnes, 2000).

Following the pioneering work of Coleman Griffith, psychology was missing in baseball until the 1950s when the St. Louis Browns hired sport psychologist David Tracy. The owners agreed and felt that if psychologists were being used in other industries to improve performance, they could also be used to help professional baseball teams (Kornspan \& MacCracken, 2003). One of the first responsibilities Tracy had was to convince the Browns players that learning psychological skills could improve their performance. As to be expected, some players were apprehensive about working with him because they assumed he was there to treat them for psychological or clinical issues (Tracy, 1951).

Although Tracy implemented a number of psychological interventions with Browns players, hypnosis was the most commonly discussed intervention by local and national media 


\section{AN EXPLORATORY INVESTIGATION OF BASEBALL COACHES’ ATTITUDES}

(Kornspan \& MacCracken, 2003). In fact, most of his work was focused on helping the team overcome their fear of crowds and learning how to play relaxed. He helped them take control of their thought patterns, taught them relaxation techniques, and instructed them on how to reject all negative thoughts and think confidently. However, there was some confusion amongst players regarding Tracy's role as a sport psychologist. One suspected reason for this was the uncertainty surrounding the exact role and responsibilities he had within the organization. For example, some of the players were unsure how he could help them perform better, believing that hypnosis was the only intervention available. Since none of the players or coaches had any previous experience working with a sport psychologist and researchers knew very little about the relationship between the mind and performance, it is easy to understand how confusion may arise. Despite the advancement in evidence-based interventions through improved science and technology, lack of awareness and misunderstanding of sport psychology, both barriers to effective consultation, could still impact the relationship between SPCs and baseball players.

The reaction to Tracy's work with players was mostly positive, and the Browns president at the time admitted that he was received favorably by most of the players. After his experience with the Browns, Tracy believed that sport psychology would have a role in baseball and SPCs would be an added resource for most Major League teams. Perhaps more importantly, he believed that a psychologist could play a vital role in working with athletes and sports teams (Tracy, 1951). Despite the encouraging predictions, available public record indicates three decades passed before other MLB teams entertained the idea of utilizing sport psychology services. 
AN EXPLORATORY INVESTIGATION OF BASEBALL COACHES’ ATTITUDES

\section{The Use of Sport Psychology in Baseball: The Modern Era}

Despite the favorable response from team ownership after Griffith and Tracy's work with the Chicago Cubs and St. Louis Browns, it remains unclear whether psychologists, SPCs, and other SPPs have become an integral or accepted component of modern baseball culture (Kornspan \& MacCracken, 2003). While their work did lay the foundation for "the mental game" to become an often-talked about topic among baseball players and coaches, the relationship between the two has yet to be empirically researched and still appears to be misunderstood. As stated previously, it appears several amateur, college, and professional teams have or currently work with SPPs, but there is no evidence or research looking at the relationship between sport psychology professionals and baseball's decision-makers: the coaches. This dichotomy is interesting considering the emphasis baseball coaches place on the importance of developing the mental game.

In spite of the paucity of evidence-based research on sport psychology and baseball, there are a plethora of popular media articles, websites, and books outlining how the psychological aspects of baseball contribute to athletic performance. Publications like The Mental ABC's of Pitching, The Mental Game of Baseball, Heads Up Baseball, and The Mental Keys to Hitting are all must-haves for baseball players looking to improve their psychological skills and gain a deeper understanding of the impact the mind can have on performance.

Interestingly, Charles Maher, current sport psychologist for the Cleveland Indians and author of The Complete Mental Game of Baseball (2011), suggested sport psychology is an integral component of performance at the Major League level. In a recent book, he emphasized that some of his best work is done with the Indian's coaching staff, perhaps because they can have a direct impact on player's mental health. Similarly, George Gmelch (2006) indicated that 


\section{AN EXPLORATORY INVESTIGATION OF BASEBALL COACHES’ ATTITUDES}

in recognizing the impact a players' mental makeup could have on performance, most baseball organizations now ask potential prospects to take a psychological test such as the Athletic Motivation Inventory. Maher and Gmelch believe that has been a shift in the philosophy of some MLB organizations over the past decade, and contend that teams in all professional leagues, including every Major League team, will soon be utilizing sport psychology services. If that is the case, sport psychology will have to first become a widely accepted and understood field of study, much in the same way modern science and technology has positively impacted the field of strength and conditioning.

To help corroborate Maher and Gmelch's hypothesis, the head researcher has firsthand evidence that a number of SPPs have worked with Major League teams and players over the past 15 years. For example, the following SPPs currently work or have worked with MLB teams in some capacity over that time span: Fran Pirozzolo (Texas Rangers, New York Yankees), Ken Ravizza (Anaheim Angels, Tampa Bay Rays), Charles Maher (Cleveland Indians), Geoff Miller (Pittsburgh Pirates, Atlanta Braves), Jason Selk (St. Louis Cardinals), Bernie Holliday (Pittsburgh Pirates), Seth Kaplan (Baltimore Orioles), Lance Green (Tampa Bay Rays), and Rob Siefer (Florida Marlins). In the past 15 years, at least twelve Major League Baseball teams (40\%) have used sport psychology services.

The primary researcher has been in personal communication with all but one of the SPPs in the preceding paragraph and interviewed them firsthand about their roles, responsibilities, and relationships with the players, coaches, and management in those organizations. Results revealed a wide range of roles and responsibilities depending on structure of the organization and level of employment between the SPP and organization (i.e., full time vs. part time), indicating there is no true "blueprint" or "model" for organizations to follow with regard to utilizing sport 


\section{AN EXPLORATORY INVESTIGATION OF BASEBALL COACHES’ ATTITUDES}

psychology services. Interestingly, the SPPs have diverse educational backgrounds and training experiences. For example, three are clinical psychologists, two have extensive experience with the US Army, and at least two lack any formal education in sport science or sport psychology. It appears that organizations may be looking for a unique skill-set or set of experiences based on their philosophy, approach to player development, or definition of "sport psychology". This inconsistency may confuse baseball coaches about who may be a "good fit" for their program or organization, further contributing to the misunderstanding towards sport psychology throughout baseball culture.

\section{Attitudes Towards Psychology and Sport Psychology Services}

\section{Factors that Impact Help-Seeking Behaviors}

Psychology is a field of study defined as "the science of behavior" or "the study of mind and behavior in relation to a particular field of knowledge or activity (www.mirriamwebster.com, 2013). The widely accepted view of professionals who work in psychology is that they help people to maintain an overall balance of mental and emotional health and strive to maintain a positive well-being. The science and practical application of studying psychology and the interaction between the mind, body, and the world around us really came into focus after World War I and World War II when thousands of GIs returned home in need of help dealing with the sights, sounds, and experiences of war (Benjamin, 1986). Over the past 75 years, interest in seeking out psychological help has been inconsistent, perhaps because people did not understand what psychologists do or how they could be helpful (Neelis, 2008).

Previous attempts to investigate students' attitudes towards psychology have been done by examining how people decide to engage in help-seeking behaviors (Masuda et al. 2005). Seeking out help from professionals can be an intimidating process where one inherently admits 


\section{AN EXPLORATORY INVESTIGATION OF BASEBALL COACHES’ ATTITUDES}

he or she has a weakness and cannot fix or improve their circumstances on their own. These thoughts could lead to feelings of inadequacy, apprehension, uncertainty about the counseling process, and an urge to keep their help-seeking behaviors private (Lambert, 2007). The same study found people using counseling services had little support from family, remained confused about proper help-seeking terminology, felt that others assumed help-seekers are weak-minded individuals, and experienced the stigma and negative connotations some members of society proscribe to counseling. Research also suggests that gender roles heavily impact an individual's relationship with psychology and help-seeking behaviors. For example, study after study indicates females have more positive attitudes towards psychology, past experiences with psychologists can better predict help-seeking behaviors, and prior experience results in a more positive attitude towards seeking help (e.g., Masuda, Suzumura, Beauchamp, Howells, \& Clay, 2005). In both studies, subjects were happier after seeking help, demonstrating the value in reaching out to professionals trained in the science of helping people become happier and improve their well-being (Lambert, 2007).

Age may also be a factor contributing to one's decision to seek help-seeking services. For example, Martin (2005) identified potential barriers that may deter children and adolescents from seeking help are fear of increased psychological stress, fear of breach of confidence, and the belief that counseling will not be helpful; these fears may exist in athletes as well. In general, research has found adolescent athletes view the counseling process less positively than adults and people become more accepting of psychological help as they age (Esters, Cooker, \& Ittenbach, 1998; Garland \& Zigler, 1994). Since coaches in the current study varied in terms of age, coaching experience, and competition level coached, it could be useful to understand how these factors impacts help-seeking behavior. 


\section{AN EXPLORATORY INVESTIGATION OF BASEBALL COACHES’ ATTITUDES}

Despite trends in the relationship between age and attitudes towards psychology, researchers have determined these attitudes are fluid and may change with experience or education. For example, in one study examining perceptions of psychological problems and attitudes about seeking help, researchers asked 40 adolescents ages 13-17 to complete surveys about mental health and had the treatment group $(n=20)$ complete an instructional unit related to psychological health (Esters et al., 1998). Both groups were then asked to complete a postinstructional survey, and results indicated that the interventions were successful in improving participant's attitudes towards seeking help for personal problems. The results of a postevaluation three weeks later maintained significance and participants attitudes towards mental illness began to closer mirror those of mental health professionals (Esters et al., 1998). Since adolescents are more reliant on adults, Esters et al. (1998) believe that they will be more likely to ask for help than a young adult entering college. Anderson and colleagues (2004) explored the potential relationship between age and help-seeking behaviors by splitting 112 athletes into two groups: younger than $20(n=39)$ and older than $20(n=73)$. They found that specific age cutoff was not a significant influence on athletes' attitudes toward seeking sport psychology services (Anderson et al). The results did not confirm their hypothesis that age would significantly impact athletes' attitudes towards sport psychology, however there may have been useful to further examine differences in middle school (i.e., ages 10-14), high school (i.e., ages 14-18), and college athletes. Research exploring why attitudes towards psychology have been inconsistent suggests that consumers and potential clients remain unclear about how psychologists can help people improve quality of life, and are unsure how psychological services can help (Benjamin, 1986). 


\section{AN EXPLORATORY INVESTIGATION OF BASEBALL COACHES’ ATTITUDES}

Coaches are in a unique position to inform athletes of all ages how mental training can be helpful, and in turn, athletes may be more inclined to make it a component of their training. Without dialogue about mental training or psychological skills with their coaches, athletes may not know about the potential benefits of sport psychology (Ravizza, 1998). Interestingly, research on college student-athletes found they had a slightly more positive attitude towards sport psychology services than high school athletes (Martin, 2005). This could be due to the increased demands of collegiate athletes or the higher exposure rate to SPCs in high school than college. It may also reflect a higher general willingness to ask for help by athletes compared to the rest of the population; athletes are encouraged from an early age to seek feedback from coaches when there is room for improvement. However, Watson (2006) recently identified barriers to college students seeking services included struggles with time management issues and the fear associated with what others may think of them. These concerns and inconsistent findings may or may not inhibit students from seeking services, and further research on impact of age on help-seeking behavior is warranted.

In the case of baseball, one would presume that if sport psychology was an accepted part of baseball culture, coaches and athletes would not flinch at the idea of working with an SPP. Despite the fact that society appears to be more accepting of counseling than in the past (Watson, 2006), SPCs need to be more aware of what coaches and athletes think about sport psychology services and help clients better understand how they can utilize sport psychology services.

\section{Attitudes Towards Sport Psychology}

Sport psychology is defined as the scientific study of people in sport and the application of the knowledge gained. This is accomplished by understanding the psychological factors which affect the physical performance of individuals and determining how one's participation in 


\section{AN EXPLORATORY INVESTIGATION OF BASEBALL COACHES’ ATTITUDES}

sport impacts their development and overall well-being (Weinberg \& Gould, 2011). The current status of sport psychology through the public eye remains unclear. While sport participants certainly acknowledge the role the "mental game" plays in performance, using the term "psychology" in a sporting context could rub some people the wrong way. The general public, including coaches, athletes, and others professionals who work in and out of sport, sometimes view those who work with sport psychology consultants (SPC) as "head cases" or "mentally weak" (Ravizza, 1998), in turn, making them less willing to utilize sport psychology services. This phenomenon has more to do with how some people perceive help-seeking behaviors and less to do with the potential help and SPC may provide; which is somewhat unfortunate considering help is often a simple phone call away. The term "psychology" can have a negative connotation to some, and may foster visions to of lying down on couches and retelling stories from one's childhood. While relatively inaccurate, these associations are real challenges that SPCs face when working with athletes and coaches, prohibiting them from providing expertise and help when it could be useful.

Prior to the 1950s, coaches had very little access to education or resources about the psychological and social factors associated with sport and performance. Up until that point, supplementary education for coaches focused on biomechanics and physiology (Cratty, 1973). In the 1960s, many coaches' focus and interest turned to understanding the impact psychology has on performance. Scientists in Europe pioneered much of the early research on sport psychology, and though the information laid the groundwork for evidence-based practice, it primarily addressed psychology and motor learning theory, not necessarily the application of sport psychology principles (Kozar \& Mechikoff, 1983). Subsequently, a shift in the approach to research and application of sport psychology concepts had to occur if the field was going to 


\section{AN EXPLORATORY INVESTIGATION OF BASEBALL COACHES’ ATTITUDES}

develop any traction or relevancy in the extremely competitive and world of sports. In response, the past 30 years have seen incremental growth in the field, a larger increase in the utilization of SPPs by Olympic and professional athletes, and a greater interest in the practical application of sport psychology theories to help improve performance (Tod, 2007).

SPCs or mental skills coaches typically work from as strengths-based perspective, whereas clinical psychologists generally work from a deficit or "improving weaknesses" model. Due to this lack of understanding, some SPCs market themselves as "mental skills coaches" or "performance enhancement consultants" in order to dissociate with the term "psychology", ostensibly limiting such stereotypes before they even have a chance to be made. This shift in approach may yield more positive perceptions with athletes and coaches, thus resulting in more sport participants seeking help from sport psychology professionals without fear of judgment or ridicule from their peers. However, further research must be done to better understand the impact factors like terminology and marketing strategies may have on utilization of sport psychology services.

\section{Measuring Athletes' Attitudes Towards Sport Psychology}

Sport psychology professionals need to continually examine athletes and coaches (i.e., clients) attitudes, perceptions, and willingness to use sport psychology services (Zakrajsek et al, 2011). There have been a number of attempts to examine the attitudes, perceptions, and experiences of athletes' at all age groups with sport psychology professionals. These studies have attempted to bridge the gap between athletes and sport psychology professionals, in hopes of determining how to better serve athletes and market services more effectively. Examining research in this area is important because athletes' beliefs and expectations can often mirror that of their coaches. Examining athletes' and coaches' attitudes, expectations, and previous 


\section{AN EXPLORATORY INVESTIGATION OF BASEBALL COACHES’ ATTITUDES}

experiences with sport psychology services can also help practitioners understand how to gain better entry with teams and identify potential clientele. Finally, this knowledge could be valuable for SPCs because of the influence of the athlete-coach relationship and the impact coaches can have on their athletes when it comes to seeking out sport psychology services (Zakrajsek et al, 2011). The information gathered by studying these factors could help SPPs gain a better understanding of why these services tend to remain underutilized with certain populations.

Researchers looking at youth athletes' attitudes towards sport psychology have found interesting results. For example, one study examining the attitudes of male and female high school athletes $(\mathrm{N}=65)$ from a rural areas suggested that teenage sport participants feel they might get made fun of on or seen as "different" if they sought out sport psychology services. More importantly, they felt it was important to have approval from their coaches to use an SPC and thought highly of having individual or group sessions with an SPC as opposed to other forms of sport psychology information (Blom, Hardy, Burke, \& Joyner, 2003). Results from this study indicate the importance of gaining a greater understanding of youth and high school baseball coaches' relationship with sport psychology.

There have also been several studies examining college athletes' attitudes and beliefs about sport psychology. For example, in their study on college hockey players, Dunn and Holt (2003) found that college athletes believe utilizing a sport psychology professional would be good for the team. Furthermore, implementing a sport psychology program without coaches present enabled the athletes to be more honest and comfortable with the SPC. Athletes in the same study suggested they liked that the SPC was easy to speak with and positive, fair and 


\section{AN EXPLORATORY INVESTIGATION OF BASEBALL COACHES’ ATTITUDES}

objective regardless of a player's skill level, emotionally invested in the players and their success, and that the SPC was trustworthy and kept confidentiality.

In a study examining receptivity to mental skills training by SPCs, researchers surveyed 2,440 NCAA Division I student-athletes to assess their willingness to seek sport psychology services, perceptions of the potential benefits of mental training for their team, and support of possible roles for an SPP at their university (Wrisberg, Simpson, Loberg, Withycombe, \& Reed, 2009). They found that females were generally more receptive to sport psychology services than males, individual and team sport athletes were interested in different types of mental skills, athletes with previous sport psychology consulting experience were more open to those with none, and athletes with highly effective past experiences were more receptive than those with less effective experiences. These findings provide several important insights for SPCs providing services to NCAA student-athletes.

Studies on elite athletes have indicated that an effective mental skills training program should begin two or three years prior to the Olympics to ensure optimal preparation (Orlick \& Partington, 1987). Results from the same study found that attitudes are positive when the SPCs is willing to work individually with the athlete, invested time and energy with them beyond the original session, and were genuinely interested in their well-being (Orlick \& Partington). In a qualitative study assessing 30 elite English athletes' experiences with sport psychology, Anderson, Miles, Robinson, and Mahoney (2004) found athletes' attitudes are more positive when SPCs are personable, have above-average communication skills, and can effectively teach and apply sport psychology principles. 


\section{Attitudes Towards Seeking Sport Psychology Consultation Questionnaire}

One of the first major attempts to develop a quantitative instrument to understand a perceptions and attitudes towards sport psychology services was done by Martin and colleagues (1997) and looked specifically at college athletes. The study resulted in the development of the Attitudes Toward Seeking Sport Psychology Consultation Questionnaire (ATSSPCQ). The instrument was a refined version of the questionnaire developed by Wrisberg and Martin (1994), who compiled a list of test items that sought to explore athletes' attitudes about sport psychology and was developed by combining important elements in sport psychology and counseling literature. Based on existing inventory content from previous sport psychology studies (e.g., Gould et al., 1987) and counseling psychology research (Fischer \& Turner, 1970; Tinsley, 1982), the following five constructs were developed and operationally defined to be included in the original instrument: a) stigmatization, b) recognition of need, c) confidence in sport psychology, d) social desirability, and e) interpersonal openness. The researchers surveyed 284 Caucasian and African-American male and female athletes at a major southeastern university and received 230 usable questionnaires ( $81 \%$ response rate) to develop the ATSSPCQ. The results of the factor analysis indicated that there are at least three dimensions underlying the attitudes of NCAA Division 1 student-athletes towards seeking sport psychology consultation (Martin et al., 1997). The analysis of items that loaded on each factor showed three constructs: a) stigma tolerance (sport psychology and sociocultural), b) confidence in sport psychology consultation/recognition of need, and c) personal openness/openness to sport psychology consultation that influenced athletes attitudes and willingness to use sport psychology services (Martin et al., 1997). 
AN EXPLORATORY INVESTIGATION OF BASEBALL COACHES’ ATTITUDES

\section{Sport Psychology Attitudes-Revised}

In order to further the understanding on this topic, researchers continued to examine the validity and reliability of the ATSSPCQ. Considering the relationship between perceptions and help-seeking behaviors, there have been several attempts to develop a valid and reliable instrument to assess athletes' attitudes towards services. Based on an examination of the literature in counseling and sport perception research, Martin and colleagues (1997) developed a theoretical assessment of athletes' attitudes towards sport psychology consultation. These attempts led to the development of a second instrument, the Sport Psychology Attitudes Revised (SPA-R) (Martin et al., 1997, 2002). In their questionnaire, Martin et al. (1997) developed and operationally defined the following important constructs of attitudes toward help-seeking behavior: stigmatization, recognition of need, confidence of sport psychology, social desirability, and interpersonal openness. These items were then developed to correspond with the theoretical definition under each construct (Zakrajsek \& Zizzi, 2007). Exploratory and confirmatory factor analysis procedures with over 1500 athletes from the United States, United Kingdom, and Germany showed a four-factor solution of athletes' attitudes toward seeking sport psychology consultation (Martin et al., 2002).

The Sport Psychology Attitudes-Revised (SPA-R) instrument is a 25 item four factor solution of athletes' attitudes: stigma tolerance (expected negative consequences of seeking consultation); confidence in sport psychology consultation (belief that consultation and mental training is useful); personal openness (the willingness of the respondent to try consultation and mental training); and cultural preference (identity with own nationality, ethnicity, culture, or race). The test-retest reliability and internal consistency (Cronbach's alpha) were found to be acceptable. The SPA-R has been shown to be a valid and stable measure of athletes' attitudes 


\section{AN EXPLORATORY INVESTIGATION OF BASEBALL COACHES’ ATTITUDES}

towards seeking consultation. Further work examining how the instrument could assist SPCs in measuring athletes' interest and receptiveness to using services has shown that confidence in consulting, perceived behavioral control, and subjective norms were found to be independent predictors and accounted for nearly $40 \%$ of the variance in athletes' intentions (Anderson, Hodge, Lavallee, \& Martin, 2004). These results confirm the suggestion that measuring athletes' attitudes can provide helpful information regarding intentions to use services.

\section{Measuring Coaches Attitudes Towards Sport Psychology}

Although athletes serve as main consumers of sport psychology services, coaches are often in the position of hiring SPPs, and their attitudes and opinions about sport psychology may filter down to their athletes (Zakrajsek \& Zizzi, 2007). Despite the importance of research on coaches' and sport psychology, research on athletes' attitudes and how they relate to intentions to use sport psychology services is much more prevalent compared to research with coaches (Zakrajsek \& Zizzi, 2007). Coaches can impact their athletes in various ways, and part of their job is to teach techniques and tactics that can help change athlete's behaviors to improve their skill level and enhance performance (Martin \& Lumsden, 1987). They can influence their athlete's behaviors through helping them develop specific characteristics and skills like motivation, competitiveness, and maturity (Giacobbi, Roper, Whitney, \& Butryn, 2002), and communicating the importance of developing various social and psychological skills (Gould, Chung, Smith, \& White, 2006). Since one of the primary purposes of sport psychology interventions is to improve performance through developing mental skills, utilizing sport psychology techniques can be a way that coaches can help modify behavior and improve performance. 


\section{AN EXPLORATORY INVESTIGATION OF BASEBALL COACHES’ ATTITUDES}

Coaches seem to be aware that athletes look to them for guidance. Gould et al. (2006) found that coaches do realize the influence they have over their athletes, and this impact can play a big role in how athletes' view sport psychology and mental skills training. Because athletes are likely to follow the guidance and advice of their coaches, it is crucial for coaches to understand the role they play when it comes to an athlete's decision to utilize sport psychology services. Therefore, research on coaches' attitudes must continually be examined to further bridge the gap between theory and practice.

Previous studies on coaches have shown that those who work with SPPs are more informed on the purposes of sport psychology than those who do not (Sullivan \& Hodge, 1991). Coaches have also previously reported they are willing to work with an SPC and suggest that sport psychology could help promote athletic development (Silva, 1984). However, much of the previous research in this area has been qualitative and only examined elite coaches (Zakrajsek, Martin, \& Zizzi, 2011). Although limited, these studies have attempted to bridge the gap between athletics and sport psychology professionals, in hopes of determining how to better serve athletes and market services more effectively.

Some research on coaches and their understanding of the mental game is somewhat curious. For example, Harris and Harris (1984) found that coaches may attribute losses to emotional and mental aspects of life, however, they spend little time actually developing athletes' mental skills. Similar research suggests that although coaches tend to attribute poor performance to the mental aspects of the game, they generally see it as a physical breakdown in athlete performance (Figone, 1999). The same study found coaches believe they lack the time and education of how to teach athletes psychological skills that can improve performance (Figone, 1999). Consequently, coaches have their athletes practice more on the physical aspects 


\section{AN EXPLORATORY INVESTIGATION OF BASEBALL COACHES’ ATTITUDES}

of the game, continuing to neglect the original reason for poor performance: breakdown in the mental game.

Coaches' concerns about lack of education may in fact hold merit; although coaches tend to use psychological skill terms such as "relax", "confidence", and "concentration", they often assume their athletes can make the mental adjustments necessary to impact performance. Conversely, they may not know exactly how to communicate their demands effectively. A coach's verbal instruction, such as "relax at the plate", might fall on deaf ears considering the lack of practice time dedicated to learning how to relax and the reality that coaches may not know how to teach their athletes such skills. Results from one study indicated coaches' did not feel they had enough knowledge about sport psychology, they practiced sport psychology in their programs, and the majority of coaches indicated they would be interested in having an SPC work with their athletes (Sullivan \& Hodge, 1991). To summarize, coaches' report that they do not have the time or knowledge to teach mental skills, consistently use terms that their athletes may not understand, assume athletes can make changes without their help, and are inconsistent in their attempts to explain poor performance. Although these results are not representative of all coaches, the implications from these results are alarming. Therefore, a clearer understanding of coaches' what coaches think about sport psychology could be useful for coaches, athletes, and SSPs.

Much of the research on coaches' attitudes towards sport psychology has been completed outside the US. For example, Pain and Harwood (2004) used a mixed methods approach to evaluate English soccer coaches' knowledge and perceptions of sport psychology. Through survey data and interview methods with National coaches $(n=8)$, academy directors $(n=21)$, and youth academy coaches $(n=27)$, the found that a general lack of knowledge about sport 


\section{AN EXPLORATORY INVESTIGATION OF BASEBALL COACHES’ ATTITUDES}

psychology underpinned some of the most basic barriers to SPPs. Further, coaches were not clear on what services sport psychologists provide, had negative perceptions of SPPs, were not sure how to integrate sport psychology with their team, and reported the biggest barrier was to services was that they lacked proper finances to utilize an SPC.

Partington and Orlick (1987) interviewed Canadian Olympic coaches to better understand their perception and opinions about what SPCs can do for them. Results from the study found that coaches typically reported their experiences working with SPCs as positive, and that SPCs were confident in their ability to work with coaches and athletes without being intrusive. Similarly, Sullivan and Hodge (1991) found that out of 46 New Zealand coaches, almost 95\% felt that athletes would perform better if SPCs completed a mental training program with their athletes. Therefore, it could be beneficial for coaches to communicate their previous experiences with their peers to foster a clearer understanding of the purpose of sport psychology across the profession. These results suggest that some coaches have accurate knowledge about specific aspects of the consultation process and services. However, there still may be a divide between what coaches know and believe about sport psychology and what SPPs can offer coaches and athletes. Although these studies shed light on coaches' attitudes towards sport psychology, they are over twenty years old and done outside of the United States. Therefore, it would be helpful to have some more recent studies of coaches from American sports.

In addition to research on coaches' attitudes done outside the United States, several attempts have been made to evaluate the use of sport psychology professionals at American universities. For example, Voight and Callaghan (2001) administered questionnaires to 115 Division I universities to determine the number of schools using sport psychology services. Results indicated that 51 of the 96 schools that returned the survey utilized some form of sport 


\section{AN EXPLORATORY INVESTIGATION OF BASEBALL COACHES' ATTITUDES}

psychology services, and that of the 10 different sport psychology positions at those universities, the most frequent was a part-time consultant hired by a specific sport program. In another study, Walker and Eslinger (2003) examined sport psychology attitudes of 50 NCAA Division I ( $n=37)$ $\&$ II $(n=13)$ coaches through a self-administered survey. They found that coaches generally have a positive outlook towards sport psychology and older coaches were more likely to utilize sport psychology services than their younger counterparts.

Wrisberg, Loberg, Simpson, Withycombe, and Reed (2010) examined 815 NCAA coaches' experiences and attitudes toward sport psychology. Specifically, they researched their willingness to encourage their athletes to see an SPP, their support of three possible roles for an SPP at their institution, and their willingness to seek mental training for those who had access to an SPP at their institution. Results indicated that coaches were more willing to refer their athletes to SPCs for performance-related issues than personal concerns. The authors also found that coaches with current access to an SPP were interested in mental training for improving performance and were more apt to seek out services if they had more contact with the SPC and thought they were effective. Of the coaches surveyed who have access to an SPP, they seemed to be willing to encourage their athletes to seek assistance from SPP, and their receptivity is increased if the coach has been in contact with the SPC more than five times and perceives s/he to be effective (Wrisberg et al., 2010). Despite several attempts to quantitatively assess coaches' attitudes towards sport psychology in the early 2000s, a reliable and valid measurement for researchers interested in this area of study had yet to be created.

\section{Sport Psychology Attitudes Revised - Coaches}

In response to a gap in the literature using quantitative methods to assess coaches' attitudes and no widely accepted evidence-based instrument to survey them, researchers were 


\section{AN EXPLORATORY INVESTIGATION OF BASEBALL COACHES’ ATTITUDES}

determined to develop a valid and reliable instrument that taps into their attitudes towards sport psychology services (Zakrajsek and Zizzi, 2007). In turn, the Sport Psychology AttitudesRevised Coaches (SPA-RC) was developed in 2007 and has been used with coaches since then. The SPA-RC was adapted from the SPA-R, originally designed by Martin and colleagues (2002) to research athletes' attitudes towards sport psychology. In the first attempt to validate the development of the SPA-RC, Zakrajsek and Zizzi examined collegiate swimming and track coaches $(\mathrm{N}=374)$ found confidence in the SPC to be the most significant predictor of coaches' intentions to use sport psychology services. This was followed by previous exposure, and expectations of the consultation process (Zakrajsek \& Zizzi, 2007). The authors identified the following areas of inquiry as important to address when examining coaches attitudes towards sport psychology: stigma tolerance, confidence in sport psychology consultation, personal openness, and cultural preference. However, because $74 \%$ of their sample worked with male and female athletes from non-contact sports, the authors suggested future research should examine coaches who work primarily with athletes from one gender and various sports. The current study would fill both gaps in the literature and help further solidify what coaches' think about sport psychology.

In a recent study using the SPA-RC examining 235 Texas high school football coaches' attitudes toward sport psychology consultation, Zakrajsek, Zizzi, and Martin (2011) found that only three percent had previously used sport psychology. Additionally, $62 \%$ had no intention of using services within six months after participating in the study. These results are particularly interesting in the context of this study, since football is a sport with a culture somewhat similar to baseball (i.e., masculine). Unlike baseball, however, there is less evidence to suggest that football coaches reference the "mental game" as often as baseball coaches in popular media 


\section{AN EXPLORATORY INVESTIGATION OF BASEBALL COACHES' ATTITUDES}

articles and interviews. Additionally, high school football coaches might not have the same level access to SPCs as baseball coaches, and the geographic location of the sample may have contributed to the results.

\section{Factors Impacting Sport Psychology Services and Professionals}

\section{Stigma Tolerance}

Not all research on attitudes and perceptions of sport psychology has been positive. One challenge facing SPPs is the negative association that coaches and athletes may associate with sport psychology. The stigma may be a reflection of cultural norms, personal experiences, sportspecific attitudes towards help-seeking behavior, or subjective perceptions, where any combination of those factors may significantly impact one's relationship with sport psychology. Specifically, some coaches may think that SPCs only work with athletes who are "head cases" or “problem athletes” (Ravizza, 1988). Based on social norms, lack of knowledge, and sport culture, some coaches and athletes may believe SPPs are "shrinks"; a negative stereotype that would negatively impact even the most confident of athletes. Furthermore, "sport psychology" may be linked to the term "psychology", a word that generally implies "fixing a problem", and very few athletes have the desire to be labeled a "mental case".

Interestingly, researchers have found that in some instances, athletes may be aware of the potential benefits sport psychology and the impact it can have on performance, yet consciously decide to avoid it altogether (Ferraro \& Rush, 2000). In their study of twenty male $(n=17)$ and female $(n=3)$ athletes from New York City, Ferraro \& Rush (2000) found that although 100\% of participants indicated they would benefit from seeing an SPP, only two had previously utilized sport psychology services. Athletes reported the biggest barriers to seeking services were lost time and money. However, when asked about how an SPP could be helpful, none of the 


\section{AN EXPLORATORY INVESTIGATION OF BASEBALL COACHES’ ATTITUDES}

participants referenced any of the emotional components that impact performance and opted to mention more objective principles like visualization and concentration. Based on this finding, the authors hypothesized that the participants may not be willing to recognize the impact one's emotions may have on performance.

Previous studies have suggested that males tend to have a more negative attitude on stigma towards help-seeking individuals and services than females, both in athletics and the general population (e.g., Buckley \& Malouff, 2005; Martin, Wrisberg, Beitel, \& Lounsbury, 1997; Morrison, De Man, \& Drumheller, 1993). More specifically, women and those with higher levels of education may have more tolerance and less socially restrictive attitudes toward help-seeking individuals. Martin et al. (1997) also reported the females tend to feel less of a stigma tolerance towards SPPs, potentially due to societies' portrayal and assumptions about masculinity and femininity and defined gender roles of sport. Similarly, Martin (2005) found athletes in contact sports (e.g., football, basketball) were more likely to stigmatize sport psychology than athletes from non-contact sports (e.g., golf, tennis). Specifically, athletes from football and wrestling were, both dominated by males, had the highest stigma towards sport psychology services. Finally, Zakrajsek and Zizzi (2007) found male coaches had a higher stigma towards utilizing SPPs and that adhering to male gender roles may impact athletes' use of SPCs. The higher stigma towards help-seeking behaviors by males may result in the fear that they will be emasculated or perceived as weak by their peers after utilizing services.

\section{Openness towards Sport Psychology Services}

In addition to the impact of stigma tolerance on utilization of sport psychology services, researchers have examined openness to sport psychology professionals as a reason coaches and athletes may or may use SPCs. For example, Zakrajsek and Zizzi (2007) found a correlation 


\section{AN EXPLORATORY INVESTIGATION OF BASEBALL COACHES’ ATTITUDES}

between openness to use sport psychology services and expectations. Conversely, some coaches may not be open to working with an SPP, believing that the interaction between the athlete and SPC would undermine their authority (LaRose, 1988; Silva, 1984).

Several researchers have reported that factors such as gender, race, ethnicity, and age may contribute to athletes' openness towards sport psychology services (Anderson, Hodge, Lavallee, \& Martin, 2004; Brooks \& Bull, 1999; Gould, Dieffenbach, \& Moffett, 2002). Anderson et al. (2004) found despite the fact that New Zealand athletes were generally receptive towards SPPs and had little problem utilizing sport psychology services, males and those without prior experience were less positive towards sport psychology services. Martin and Colleagues (1997) also determined that factors such as gender and race contribute to athletes' openness towards sport psychology, supporting previous research on gender differences in help-seeking behaviors. Similarly, results from two previous studies (Brooks \& Bull, 1990; Martin, Wrisberg, Beitel, \& Lounsbury, 1997) suggested females are more open and have less stigma tolerance towards sport psychology than male athletes. Brooks \& Bull (1990) also found females to be more accepting of teammates who seek out sport psychology services, corroborating previous findings in gender differences and help-seeking behaviors. Despite these barriers, coaches must keep in mind that in order for athletes and teams to reach their potential, they must be prepared physically, strategically, and psychologically (Martin \& Lumsden, 1987).

\section{Perceptions of Effectiveness, Expectations, and Experiences with Sport Psychology Services}

Depending on one's previous experience with sport psychology services, they may already have an assumption about the effectiveness of SPPs and the services they provide. Previous experiences with counselors, psychologists, or any other help-providing professional may impact the expectations and assumptions that clients make, even prior to meeting them. 


\section{AN EXPLORATORY INVESTIGATION OF BASEBALL COACHES’ ATTITUDES}

Perceptions of effectiveness may also come from initial impressions upon meeting an SPP for the first time, where decisions and judgments could be made very quickly and impact the relationship between and SPP, athlete, and team.

Previous research on coaches and athletes have identified important factors involved in the process and effectiveness of consultation such as the onset and timeframe of services, role of the consultant, and communication patterns of the consultant, athlete, and coach (Zakrajsek \& Zizzi, 2007). Any variation in these factors may impact the extent to which an SPP could be helpful. Similarly, lightning-quick judgments about a sport psychology professional's appearance, vocal tone, body language, or overall presence could affect the consultation. These initial observations have been shown to affect the likelihood an athlete will seek sport psychology services (Lubker, Watson, Visek, \& Greer, 2005). Additionally, Lubker and colleagues (2008) found athletes were most willing to utilize services if the SPC was like them (e.g., sport experiences, gender), has appropriate credentials and training to help them improve performance, and could relate well to the athletes. In a more recent study, Lubker et al. (2012) were interested in determining the specific qualities and characteristics athletes would be most likely to look for in an SPP if interested in sport psychology services. The authors surveyed 464 college student-athletes and used a conjoint market analysis to determine the specific SPP characteristics athletes' looked for in an SPP. Results indicated athletes' would be most likely to work with a physically fit female SPC with similar characteristics (e.g., ethnicity, sport knowledge, athletic background), high interpersonal skills, and proper education and training to help the athlete improve performance. Although fourteen athletes did not indicate a specific gender, participants in the study included both male $(n=239)$ and female $(n=211)$ athletes. 


\section{AN EXPLORATORY INVESTIGATION OF BASEBALL COACHES' ATTITUDES}

Despite the relatively even distribution of males to females, it is interesting that athletes would be more likely to work with female SPP if given the choice.

Interestingly, previous research on the area of athlete's gender preferences for helpproviding professionals revealed contradictory results. For example, Wesner and Valiance (2007) found that athletes have no gender preference for working with team physicians. Additionally, in a study on collegiate athletes' gender preferences for strength and conditioning coaches, Magnusen and Rhea (2009) found that D-I football players prefer male strength coaches, whereas female athletes have no preference on the gender of their strength and conditioning coaches. Research like this can provide SPCs with a better understanding of some the subjective norms and preferences of athletes when it comes to working with help-providing professionals.

Previous research on coaches' preferences, expectations, and experience with sport psychology services has been conducted via surveys and interviews. Research on elite coaches has identified that coaches prefer SPCs who work individually with athletes, exchange and communicate ideas with the coach, and act as a facilitator and give feedback to athletes (Gould et al., 2000; Orlick \& Partington, 1987; Partington \& Orlick, 1987). Similar research has also demonstrated that coaches prefer consultants to initiate meeting for improving communication with athletes and the staff, identify individual strengths and weaknesses of athletes, advise coaches and athletes on how to cope with stress, and increase contact with coaches (Gould, Murphy, Tammen, \& May, 1991; Partington \& Orlick, 1987). In another study on coaches' preferences, researchers found that the most effective means of selling sport psychology services to coaches involved talking about how consultations worked, as well as providing some real-life stories coaches can relate to (Speed, Andersen, \& Simmons, 2005). Similarly, Zakrajsek \& Zizzi 


\section{AN EXPLORATORY INVESTIGATION OF BASEBALL COACHES’ ATTITUDES}

(2007) found that it could be helpful for SPCs to spend time talking with coaches about how to use services most effectively and what to expect with the consultation process. This would contrast a common approach of SPCs, who present coaches in their first meeting with a "menu" of various sport psychology principles, neglecting to provide them with any information regarding how they plan to work with their athletes. The current study aims to understand some of the most effective ways consultants can approach working with baseball coaches across age groups and skill levels.

Previous exposure to sport psychology may also be one of the primary variables that influence coaches' attitudes and intentions to use services. The primary means for coaches to gain exposure and knowledge to sport psychology information may include attending organized clinics or presentations, taking academic coursework, or reading books on the subject (Zakrajsek \& Zizzi, 2007). In one study, researchers found that the majority of coaches surveyed (65\%) reported having previous exposure (e.g., attending workshops, clinics, or courses) to sport psychology and $93 \%$ of coaches suggested an interest and willingness to use SPCs (Sullivan \& Hodge, 1991). Interestingly, despite the vast majority of coaches indicating an interest in working with SPCs, only $21 \%$ of coaches reported using sport psychology services, which implies that there could be existing or unforeseen barriers prohibiting coaches' from hiring SPPs.

Although previous exposure to sport psychology seems to play some role in coaches' attitudes, it appears simply providing knowledge about the field may not be enough to get coaches to implement mental skills with their athletes' or utilize them in their coaching. For example, research has demonstrated that a psychological skills workshop immediately enhanced interest and intended use of mental skills, but this intention decreased over time (Gould, Petlichkoff, Hodge, \& Simmons, 1990). Since the skills learned were not actively implemented 


\section{AN EXPLORATORY INVESTIGATION OF BASEBALL COACHES’ ATTITUDES}

into training programs, it appears that learning sport psychology content may increase awareness and interest, however, it remains to be seen if that results in coaches integrating sport psychology principles or services. In accordance with relatively low use of services (Rice, 1997), suggests that coaches may not be receiving vital information that could influence their choice to use sport psychology services. For example, despite the fact that coaches may be receiving content, they may not know how to gain access to services, the specific the consultation process, or where to receive information to find out how to use an SPP most effectively (Zakrajsek \& Zizzi, 2007). Further research into the factors limiting coaches from obtaining useful sport psychology information could help bridge the gap between some coaches and SPPs.

\section{Time Constraints and Money}

In a society motivated by results, winning is often the primary goal for most NCAA student-athletes and coaches, particularly those attending NCAA Division I institutions (Wrisberg et al., 2009). Despite the emphasis placed on winning and the role sport psychology could play in performance, time constraints and money may be the thorn in the proverbial side of coaches at any level. Restrictions on time spent with athletes and budgets often determined by administrators or athletic directors can negatively impact use of sport psychology services. Ferraro and Rush (2000) found that even though athletes were aware of the potential impact on performance, athletes did not chose to utilize services because of time and money. Similarly, in a study of 115 Division I athletic administrators, Voight and Callaghan (2001) found that "lack of funding" was the number one reason athletic departments were not using sport psychology services.

In addition to inadequate financial resources, time constraints may prohibit coaches' utilization of sport psychology services. For example, youth coaches generally have just a few 


\section{AN EXPLORATORY INVESTIGATION OF BASEBALL COACHES’ ATTITUDES}

hour of practice time per week with their athletes, much of which is dedicated to basic technique and skill development. High school coaches are restricted by institutional rules dictating how much time they can spend with their athletes based on specific point in the school year. Finally, college coaches are limited by strict NCAA regulations prohibiting coaches from interacting with their athletes more than 20 hours a week during the season and eight hours a week in the offseason. With budgets being cut in athletic departments across the country and the amount of time coaches and athletes spend together being heavily scrutinized by sport governing bodies, valueadded services like sport psychology may the some of the first expenses to be deleted from the bottom line.

\section{Social Influence and Gender Roles}

Researchers believe that gender norms, masculinity, ideologies, and gender roles limit the number of men who seek help in the general public and that psychologists' roles and responsibilities may not be clearly understood by society (Berger, Levant, McMillan, Kelleher, \& Sellers, 2005). Despite the general misunderstanding as to what psychologists do, women still appear to be more likely than men to reach out for help. For example, research has shown that men are less likely than women to turn to professional help when dealing with clinical issues such as depression or when dealing with stressful life events (e.g., O’Loughlin et al., 2011). In their study examining the role those factors play on help-seeking behavior in men, Berger et al. (2005) found that men who score higher on measures of gender role conflict and traditional masculine ideology tend to have more negative attitudes when it comes to seeking-help. Additionally, they found that younger men are less likely to seek help than older men, perhaps providing some insight in why such a negative stigma might occur with male collegiate studentathletes. Lane and Addis (2005) also suggest that factors such as 'culture;' and 'type of help' 


\section{AN EXPLORATORY INVESTIGATION OF BASEBALL COACHES’ ATTITUDES}

could affect men's willingness to seek help. Parslow and Jorm (2000) found that characteristics like age and education level might also contribute to the decision for men to seek-help from professionals. The revelation that gender roles play a part in decision to utilize psychological services is similar to previous literature examining differences in help-seeking behavior of men and women, male gender roles, and the relationship between openness to services and expectations in the general population (e.g., Good, 1995; Constantine \& Gainor, 2004).

Considering the impact of gender roles, social norms, and America's apparent collective stigma towards help-seeking behaviors, working as a counselor or psychologist may be particularly challenging. These barriers and challenges may be magnified in the myopic arena of sport, a microcosm of society where gender roles and social norms can be magnified by oldschool stereotypes and an unwillingness to change with the times. Being a help-seeking professional may be even more difficult in the sporting environment since constructs such as masculine culture, pressure to live up to gender roles, and steadfast traditions uphold the status quo. These socially influenced barriers could be particularly difficult to overcome in a sport such as baseball where 'coaching trees', for lack of better term, are commonplace in high schools, colleges, and professional organizations across the country. When baseball players retire and go into coaching, they often remain in the organization and 'branch' out in other roles. For example, a high school player may be hired by his former coach as a Freshman or Junior Varsity Coach, a college player may be hired as a hitting coach, or a professional player may become the third base coach for the Single-A team. While this is not always the case, the incestuous nature of the business keeps the 'old-boy' network in tact. This cyclic pattern is a natural consequence of the system, but can prohibit progressive thinking and the influx of new 


\section{AN EXPLORATORY INVESTIGATION OF BASEBALL COACHES’ ATTITUDES}

ideas within organizations, and may affect the extent to which sport psychology professionals are understood and accepted on the baseball diamond.

\section{Summary}

Coaches and athletes agree that the baseball is a game of both physical and mental skills. However, the currently climate between the sport and the field of sport psychology remains unclear. Although the prevalence of sport psychology professionals at the Major League and NCAA level seem to be increasing, there does not appear to be any previous research exploring how coaches' attitudes experience, or subjective norms impact their relationship with sport psychology. Research on athletes and coaches (e.g., Martin et al. 1997; Zakrajsek, et al., 2011) attitudes towards sport psychology reveal that previous experience, confidence in sport psychology consultation, and stigma tolerance may play a role in utilization of services. Research suggests sport participant attitudes towards sport psychology may be impacted by societal factors such as gender roles, social norms, mixed gender sports (e.g., track and field) and type of sport (i.e., contact vs. non-contact; team vs. individual) (e.g., Zakrajsek \& Zizzi, 2007). It is assumed that SPPs experience many of the barriers that psychologist, counselors, social workers, and others in the helping field from the general population such as time, money, and unrealistic expectations. The social influences, gender roles, and stigmatization towards helpseeking behavior could limit the likelihood of baseball coaches utilizing sport psychology services. Additionally, the perceptions and subjective norms baseball coaches have of their peers may also influence their decision or intention to utilize services. Coaches may have a large influence over their athletes' physical and mental development. Similarly, coaches' attitudes and beliefs may impact their athletes. Therefore, if they do not know how to address the "mental game", coaches and athletes could benefit from utilizing sport psychology services. 
AN EXPLORATORY INVESTIGATION OF BASEBALL COACHES’ ATTITUDES

\section{CHAPTER III}

\section{Methods}

The purpose of this chapter is to present the steps that were followed regarding the: (a) identification and description of participants, (b) measurement techniques, (c) demographics, (d) instrumentation, (e) data collection, and (f) data analysis. A descriptive and multivariate research design was used to explore the area of inquiry.

\section{Research Questions}

The four research questions considered in this study were:

1. How did factors such as coach's age, years of coaching, education level, skill level coached, access to sport psychology services, or previous experience with sport psychology relate to a baseball coach's self-reported use of sport psychology principles and services?

2. How did a coach's reported level of confidence, openness, stigma tolerance, and cultural preference relate to their self-reported intentions to use sport psychology services?

3. How did a coach's skill level coached relate to their reported stigma tolerance and openness to using sport psychology services?

4. How did a coach's previous experience with sport psychology services relate to their stigma tolerance, confidence in SP, personal openness, and cultural preference?

\section{Hypotheses}

The four hypotheses for this study were:

H1: No significant relationships would be observed between factors such as coach's age, years of coaching, education level, skill level coached, access to sport psychology services, or previous experience with sport psychology and use of sport psychology principles and services. 


\section{AN EXPLORATORY INVESTIGATION OF BASEBALL COACHES’ ATTITUDES}

H2: No significant relationships would be observed between a coach's reported level of confidence, openness, stigma tolerance, and cultural preference and a coach's self-reported intention to use sport psychology services.

H3: No significant differences would be observed between skill level coached and stigma tolerance and openness to using sport psychology services.

H4: No significant differences would be observed between a coach's previous experience with sport psychology (i.e., as an athlete or coach) and a coach's stigma tolerance, confidence in SP, personal openness, and cultural preference.

\section{Identification and Description of Participants}

The participants in this study consisted of members from two groups: (1) 2013 members of the American Baseball Coaches Association (ABCA) (ABCA Directory, 2013), and (2) all 2013 National Collegiate Athletic Association (NCAA) Division I, II, and III head baseball coaches. By contacting every member of both groups, the researchers employed a convenience and purposive sampling method. When the study was conducted, there were nearly 7,000 members in the ABCA including youth, high school, collegiate, junior college, and professional coaches from all 50 states. For the purposes of this study, the ABCA Executive Director granted the researcher permission to contact all ABCA members. Additionally, every head baseball coach of a team competing at the Division I, II, or III level was identified from an official listing provided by the NCAA (NCAA Baseball Coaches, 2013). The researcher used the official NCAA Directory, which is the most comprehensive listing available, to access the names of all NCAA head baseball coaches. At the time data was collected, there were 308 Division I, 271 Division II, and 379 Division III schools competing in baseball. The contact information and publicly available email address of all current head coaches was identified through the athletic 


\section{AN EXPLORATORY INVESTIGATION OF BASEBALL COACHES’ ATTITUDES}

department's website of each selected institution. There were approximately 7,500 potential participants, with some overlap between the two groups. Coaches who belonged to both groups were only contacted through their ABCA membership information.

After receiving approval from the WVU Institutional Review Board for the Protection of Human Subjects, all participants were contacted via email and invited to participate in this research project. Those who agreed to participate were taken to a secure website where they completed the survey. The website www.surveymonkey.com hosted an online version of the instruments used in the study. Survey research based on data collected from the Internet has been found to be a viable method of collecting data for scientific purposes (Dillman, 2007). The potential benefits of using an Internet-based method include easier access to participants, more time for participants to access the survey, less intrusion from the researchers, and greater anonymity of participants.

Each participant met the following criteria in order to participate:

1) At least one year of coaching experience at the youth, high school, college, Olympic, or professional level; and

2) Must have been a member of the 2013 ABCA directory or an NCAA

Division I, II, or III head baseball coach.

\section{Measurement Techniques}

Descriptive and multivariate approaches were used to explore baseball coaches' relationship (i.e., attitudes and experiences) with sport psychology. The Sport Psychology Attitudes Revised Coaches-2 (SPARC-2) (Zakrajsek, 2011) was used to quantitatively examine baseball coaches' attitudes and experiences with sport psychology. The survey also included items crafted by the principle investigator that explored baseball coaches' perceived social norms 


\section{AN EXPLORATORY INVESTIGATION OF BASEBALL COACHES’ ATTITUDES}

and sport psychology expectations; coaches' access, experience, and education with sport psychology; and items about participants' previous experience with sport psychology services. The quantitative results from the SPARC-2 were combined with the other items to provide indepth analysis of coaches' attitudes and experiences with sport psychology.

\section{Demographics}

A demographic set of questions based primarily on previous research in this area was administered as part of the study. Items from this section of the survey included questions about participant: age, gender, state or territory where they work, ethnicity, highest level of education, current level coaching (i.e., youth, high school, college, professional), current job title (e.g., head coach, assistant coach, etc.), total years in current position, and total years of coaching experience (See Appendix C). This information was seen as helpful to determining how participant demographic variables might impact coaches' attitudes and experiences with sport psychology.

\section{Instrumentation}

The survey instrument used was a modified version of the Sport Psychology Attitudes Revised Coaches (SPARC-2) (Zakrajsek, 2011) developed to evaluate coaches' attitudes towards sport psychology (See Appendix A). The survey that was used in this study was comprised of

five parts: (a) the SPARC-2 (See Appendix A), (b) Sport Psychology Access and Experience (See Appendix B), (c) Demographic Questions (See Appendix C), d) Sport Psychology Expectations and Baseball Norms (See Appendix D), and (e) Sport Psychology Education and Baseball-Related Questions (See Appendix E). The last section of the survey included several open-ended inquiries for coaches to provide more in-depth responses about the attitudes and 


\section{AN EXPLORATORY INVESTIGATION OF BASEBALL COACHES’ ATTITUDES}

experience with sport psychology. The survey was designed according to principles outlined by Dillman (2007).

Permission to use and modify the SPARC-2 for the purposes of this study was granted by the authors of the original instrument. The original SPARC-2 items were not altered in any way to ensure the psychometric qualities of the data were maintained. The SPARC-2 is a recent version of the Sport Psychology Attitudes - Revised Coaches (SPA-RC) (Zakrajsek \& Zizzi, 2007) questionnaire and is closely related to the Sport Psychology Attitudes - Revised (SPA-R) (Martin et al., 2002) questionnaire designed to assess athletes' attitudes. The SPA-R, (Martin et al., 2002) on which the SPA-RC is modified, was originally a 7-point Likert scale. It was changed to a 6-point scale in order to improve the reliability of responses (Zakrajsek \& Zizzi, 2007). Interpretation and scores on the SPARC-2 are obtained by averaging the responses within each subscale.

The four subscales of the SPARC-2 are: (a) stigma tolerance (7 items); (b) confidence in sport psychology consultation (8 items); (c) personal openness (5 items); and (d) cultural preference (6 items). The factors are assessed through 26 items, which are measured on a 6point Likert scale $(1=$ strongly disagree, $2=$ moderately disagree, $3=$ slightly disagree, $4=$ slightly agree, 5= moderately agree, and 6= strongly agree). Higher scores (closer to six) indicate a more negative attitude toward seeking SP consultation (stigma tolerance); a belief that SP consultation and mental training is useful (confidence in SP consultation); an unwillingness to be involved in SP consultation and mental training (personal openness); and a strong identification to nationality, ethnicity, culture, or race (cultural preference).

The four factors measured by the SPA-RC were previously identified based on exploratory factor analysis (EFA) conducted from the results of a study of track and swimming 


\section{AN EXPLORATORY INVESTIGATION OF BASEBALL COACHES’ ATTITUDES}

coaches (Zakrajsek \& Zizzi, 2007). The SPA-RC has established reliability and validity. Internal consistency (Cronbach's alpha) reported estimates have been found to be good to excellent: Stigma Tolerance (.90), Confidence in Sport Psychology Consultation (.87), Cultural Preference (.82), Personal Openness (.79) (Zakrajsek \& Zizzi, 2007). The authors used promax rotation on the original 25 items of the SPA-R to identify item clusters that were specific to attitudes of coaches toward sport psychology consultation. Items with loadings of .40 and above with cross loadings less than .30 were retained. The scores for each subscale were obtained by averaging the responses within each subscale. The questionnaire revealed a 21 -item, 3 factor solution on coaches' attitudes towards sport psychology consulting that accounted for $45 \%$ of the total variance. Factor 1 corresponded to stigma tolerance, factor 2 represented confidence in sport psychology consultation, and factor 3 represented personal openness, and factor 4 represented cultural preference. Exploratory factor analysis procedures revealed a 4-factor solution accounting for $52.5 \%$ of the response variance in coaches' attitudes.

To help the obtain a more in depth understanding of the research questions, additional items were added to the survey that pertained to baseball coaches' perceived subjective norms and expectations with sport psychology, previous experience with sport psychology, and intentions to use sport psychology services. These items were developed by the primary researcher and based on previous literature (e.g., Zakrajsek \& Zizzi, 2007). To maintain consistency throughout the survey, any items requiring a similar response in other sections were evaluated on an identical 6-point scale. There is literature to support each non SPARC-2 item developed specifically for this study. Specifically, five items were included to measure coaches' subjective norms and five items were included to measure coaches' expectations for sport psychology consultation. Subjective norms were examined based on responses to items such as 


\section{AN EXPLORATORY INVESTIGATION OF BASEBALL COACHES’ ATTITUDES}

"Other coaches at my level are using sport psychology services with their athletes" and "The coaches whose opinions I respect use sport psychology services with their athletes and teams". Expectations for sport psychology consultation was examined based on responses to items such as "Using sport psychology services might make our athletes think more during practice" and "The benefits of sport psychology are difficult to prove". Descriptive analysis was used for these items since they have not been used in previous studies or subject to prior analysis to determine their reliability or validity. They were designed specifically by the researcher to help answer the questions for the current study and were not included in the inferential analyses.

\section{Qualitative Items}

Several open-ended items were developed by the primary researcher and placed strategically throughout the study to collect information that may help further explain results from the quantitative analysis (See Appendix E). Open-ended questions allow researchers to further explore particular topics in greater depth. This can be beneficial for SPPs since no previous research has examined what baseball coaches' relationship with sport psychology (Patton, 2002). The qualitative items were not analyzed for the purpose of the current study; however, the data responses could be used in further research on coaches' attitudes towards sport psychology and may provide further insight into participants' experiences with sport psychology services and professionals.

\section{Data Collection}

Approval from the West Virginia University Institutional Review Board was obtained in advance of the administration of instruments (Appendix F). Initial data was collected through Internet response surveys. Electronic survey methods can improve efficiencies of administration of self-completed questionnaires such as elimination of paper, postage, mail out, and data entry 


\section{AN EXPLORATORY INVESTIGATION OF BASEBALL COACHES’ ATTITUDES}

costs (Dillman, 2007). However, it cannot be assumed that every participant has experience completing Internet surveys, and there was no guarantee potential participants checked their email during the data collection process. Despite these concerns, the researchers believed online data collection would yield the highest possible sample size, and ensure the greatest number of baseball coaches have access to the survey.

There is precedence for using web surveys as a means of data collection for sport psychology research. Previous studies utilizing web surveys as a means of data collection aimed at sport psychology professionals have yielded response rates ranging from, 29\% (Etzel, Watson, \& Zizzi, 2004) to 54\% (Watson, Zizzi, \& Etzel, 2006). Of the studies looking at sport participants, response rates have varied from $17 \%$ in a study directly examining Division I coaches' support and willingness to seek sport psychology services (Wrisberg, Loberg, Simpson, Withycombe, \& Reed, 2010) to less than $2 \%$ in a study where all Division I coaches were asked to forward an email with a link to the survey to their athletes (Wrisberg, Simpson, Withycombe, Loberg, \& Reed, 2009). According Dillman (2007) and other sources examined by the primary investigator, it appears the average response rate for Internet surveys is somewhere between 10$20 \%$. Using these estimates, the head researcher expected to see similar response rates.

During a phone call on November $16^{\text {th }}, 2012$, the Executive Director of the ABCA agreed to partner with the researcher on the project. The specific details of the agreement were discussed during a phone call on January $30^{\text {th }}, 2013$ where the Executive Director provided the researcher with a 2013 ABCA Member Directory. In order to insure that coaches' participation and responses remained confidential, each coach was contacted directly by email. Coaches were initially contacted through a pre-notice email from an “@WVU.edu” email address that alerted participants about the study and encouraged them to participate. Dillman (2007) suggest that 


\section{AN EXPLORATORY INVESTIGATION OF BASEBALL COACHES' ATTITUDES}

sending a pre-notice email could help guard against participants discarding the message containing the survey link.

As outlined by Creswell (2009) and Dillman (2007), a four-step procedure was used to communicate with potential participants. The four steps include: (a) sending a pre-notice email to all potential participants with a description of the study and encouragement to participate; (b) three days later, sending out the initial email to all participants with a description of the study, consent information, and link to the web survey; (c) one week later, sending a reminder email to each participant; and (d) after one additional week, sending a final email to all participants. Coaches received the initial email on February 21, 2013. Each email included a cover letter of explanation, consent form, and a link that will take each participant directly to the survey. The surveys were available via the website wwww.surveymonkey.com from February $22^{\text {th }} 2013-$ April $1^{\text {st }}, 2013$. All data was collected was collected by April 1, 2013 and the data was analyzed according to the procedures agreed upon by the research team. Once the surveys were completed, the data was collected and entered into version 21 of the Statistical Package of Social Sciences (SPSS) software.

Participant's rights and issues of confidentiality, anonymity, and potential risks and benefits were included in the informational cover letter (Appendix $\mathrm{H}$ ) and consent form (Appendix I). All subjects were provided with the opportunity to end participation or withdraw their data at any time and could refuse to answer any question without penalty. The total participation time for each subject was estimated to be approximately 15-30 minutes. Participants could complete the survey from any computer or Internet device. The only perceived risks to the participants are those associated with filling out an online survey (e.g., completing the survey in an uncontrolled environment, secure server not used). Prior to agreeing 


\section{AN EXPLORATORY INVESTIGATION OF BASEBALL COACHES’ ATTITUDES}

to complete the survey, the participant had the opportunity to read an informed consent letter. The letter described the study in detail and allowed the potential participants a final opportunity to complete the survey or choose not to do so. Completing the survey was considered provision of consent to participate. Upon completing the final section of the survey, participants were asked to voluntarily provide their contact information in case they are interested in receiving a copy of the results of the study.

The results of the survey were kept password protected on the computer of the primary investigator (PI). The PI and other members of the research team were the only people with access to view the data. The research team consisted of the PI's dissertation chairperson and four additional researchers (i.e., Edward Etzel, Ed.D., Bernie Holliday, Ph.D., Ed Jacobs, Ph.D., Vanessa Shannon, Ph.D., Sam Zizzi, Ed.D.). Team members are sport psychology professionals who have published numerous articles in sport psychology literature utilizing both quantitative and qualitative analysis.

\section{Data Analysis}

The data from the survey was entered into version 21 of SPSS. The five independent variables in this study were: (a) age, (b) years coaching, (c) education level, (d) access to sport psychology services, and (e) previous sport psychology experience as a player or coach. The

five dependent variables were: (a) stigma tolerance, (b) confidence in SP, (c) personal openness, (d) cultural preference toward SPPs, and (e) intentions to use sport psychology services.

Descriptive statistics were calculated for all of the independent and dependent variables. Mean response scores were calculated for each participant on each of the dependent variables. Correlations were computed between the participant's scores on the dependent variables and 


\section{AN EXPLORATORY INVESTIGATION OF BASEBALL COACHES' ATTITUDES}

several classification variables such as years coaching, age, previous sport psychology experience, and skill level coached to determine the presence of any covariates in the study.

Research question one, which stated: "How did factors such as age, years of coaching, education level, skill level coached, access to sport psychology services, or previous experience with sport psychology relate to a baseball coach's self-reported use of sport psychology principles and services?" was analyzed using descriptive statistics (e.g., means, standard deviations, etc.), Chi-square test for Independence, and t-tests. Research question two, which stated: “How did a coach's stigma tolerance, confidence in SP, personal openness, and cultural preference relate to their self-reported use of sport psychology principles and services?" was analyzed using a one-way multivariate analysis of variance (MANOVA). Research question three, which stated: “How did a coach's skill level coached relate to their stigma tolerance and openness to using sport psychology services?” was not analyzed due inadequate survey design. Research question four, which stated: "How did a coach's previous experience with sport psychology services relate to their attitudes toward sport psychology services?" was also analyzed using a one-way MANOVA. All research questions were analyzed using an alpha level of .05 unless otherwise stated.

To better interpret the data analysis, effect sizes reported were Phi for Chi Square, Cohen's $d$ for comparing group means, and partial eta squared for multivariate analysis. The following parameters were used to interpret effect sizes (Cohen, 1988): (a) Phi: small=0.2, medium $=.5$, large $=.8 ;$ (c) Cohen's $d:$ small $=0.1$, medium $=.3$, large $=.5$; and (d) Partial eta squared: small $=.01 ;$ medium $=.059 ;$ large $=.138$. Following the MANOVA analyses, a one-way ANOVA was conducted to examine the differences between each independent variable and the dependent variables (i.e., four subscales and intentions). When significant differences were 


\section{AN EXPLORATORY INVESTIGATION OF BASEBALL COACHES’ ATTITUDES}

found, multiple comparisons were done to determine which groups differ across each independent variable.

Previous researchers exploring attitudes towards sport psychology have attempted to develop an explanatory model to predict coaches' intention to utilize sport psychology professionals or services (Martin, Zakrajsek, \& Wrisberg, 2012; Zakrajsek, et al., 2011; Zakrajsek \& Zizzi, 2007). To further this area of inquiry, the researcher used the latest version of SPSS to run a multiple logistic regression analyses to determine the best models coaches' intention to use sport psychology services. Linear regression was used to determine the value of the dependent scale variable, based on its linear relationship with the predictor variables. In a logistic regression, all variables enter the model at the same time, and odds ratio comparisons can be made for each predictor variable and its affect on the outcome variable. Logistic regression analysis also provides the percent of total variance accounted for in the model. In reviewing the model, the researchers evaluated for problems with multi-collinearity, which may occur if two predictor variables are too closely correlated. Harrell (2001) suggested that for every predictor in the model, there should be between 10-20 subjects, so the researcher aimed to exceed those parameters. The sample population was approximately 7,500 , so the researcher anticipated exceeding minimum number of subjects necessary to run analysis.

The regression model analyzed is as follows: The survey item "intentions to use" was the outcome (i.e., dependent) variable and age, education level, years of coaching, primary job responsibility, stigma tolerance, confidence in sport psychology, cultural preference, and openness to sport psychology services were the predictor (i.e., independent) variables. 


\section{CHAPTER IV}

\section{Results}

\section{Demographic and General Sport Psychology Information}

Approximately 7,500 American Baseball Coaches Association (ABCA) members and National Collegiate Athletic Association (NCAA) head baseball coaches were contacted to participate in the survey. In total, 1,765 responded to the initial email request for participation for a $22.7 \%$ initial response rate. Of the total sample, 1,401 participants finished the entire survey, yielding a final a response rate of $18 \%$. Of the 1,401 participants in the total sample, 1,385 were male, 5 were female, and 11 did not identify their gender. Participants had a mean age of 42.55 years $(S D=12.84)$ and 18.08 years $(S D=12.25)$ coaching experience. Participant ages ranged from $20-86$ years old with the majority of participants in the $26-45(53 \%)$ range. Over $90 \%$ of the selected sample was Caucasian; over $80 \%$ had completed at least a bachelor's degree; and close to $50 \%$ of participants had a graduate level degree (e.g., master's, doctorate). (See Table 1 for more information)

The final sample included over 750 head coaches and 300 assistant coaches. Out of the 1,401 participants included in the analysis, 927 coached "youth" athletes (i.e., youth, club, or high school level), 700 coached "collegiate" athletes (i.e. Junior College, NAIA, Division I, Division II, or Division III level), and 102 coached "professional or Olympic" athletes. Coaches can often work with athletes from different age groups and skill levels, therefore the survey was design allowed participants to indicate every level of athlete they coach. Consequently, the total exceeds the number of participants included in the analysis. Some coaches did not specify gender, age, ethnicity, highest level of education, or level of coaching, resulting in missing data for several demographic variables. (See Table 1 for more information) 
AN EXPLORATORY INVESTIGATION OF BASEBALL COACHES’ ATTITUDES

Of the participants included in the sample, $81.4 \%$ said that they least had some knowledge about sport psychology (e.g., college courses, workshops, sport psychology books); $43 \%$ reported having previous experiences with sport psychology services as a player or coach. Thirty-three percent of participants reported currently using SP services and $89.5 \%$ of participants reported they would use sport psychology services in the next calendar year if they had proper financial resources. Over $85 \%$ of coaches indicated that they were familiar with sport psychology. Coaches who did not indicate familiarity with sport psychology were given a brief definition of "sport psychology" (Weinberg \& Gould, 2011) and provided with several examples where sport psychology principles could be implemented with their teams. Researchers did not assess how the participants who indicated familiarity with sport psychology actually defined the term "sport psychology". On average, coaches believed that baseball was $68 \%$ mental $(S D=$ $19.40 \%)$ and spent $24 \%$ of practice time $(S D=21.72 \%)$ focused on the "mental game". On a 6point Likert-scale ranging from 1 (strongly disagree) to 6 (strongly agree), coaches reported that it was very important for their athletes to have strong mental skills $(M=5.84, S D=.71)$. (See Table 2 for more information)

\section{SPARC-2, Sport Psychology Expectations, and Subjective Norms}

Descriptive analysis of the SPARC-2 items revealed that the sample of ABCA members and NCAA head coaches reported a low score on stigma tolerance $(M=1.78, S D=.77)$, indicating positive attitudes towards SP services. Coaches reported a moderately high score with regard to confidence in SP consultation $(M=4.47, S D=.77)$, which suggests a modest level of confidence in SP consultation. Participants reported a slightly higher than midpoint score on personal openness $(M=3.13, S D=.86)$, indicating they may be less than willing to participate in SP services or mental training. Finally, participants reported a moderately low score on cultural 


\section{AN EXPLORATORY INVESTIGATION OF BASEBALL COACHES' ATTITUDES}

preference $(M=2.25, S D=.95)$, indicating a weak personal identification with nationality, ethnicity, culture, or race. If a participant failed to answer specific items on a subscale but completed the SPARC-2, their score for that subscale was determined by averaging the answered items completed in that subscale. (See Table $3 a$ and Table $3 b$ for the descriptive statistics of each subscale across several independent variables)

The following results represent descriptive analyses from non-SPARC-2 items exploring coaches' subjective norms and expectations of sport psychology consultation. To maintain consistency throughout the survey, these items were evaluated on an identical 6-point scale (i.e., $1=$ strongly disagree $-6=$ strongly agree). On items addressing subjective norms, coaches slightly agreed that they had access to SP services $(M=4.0, S D=1.37)$, that coaches they respect used SP services, $(M=4.15, S D=1.33)$, and that coaches on their staff would support the use of SP services $(M=4.69, S D=1.15)$. Also, coaches reported being unclear as to whether or not their peers utilized sport psychology services $(M=3.17, S D=1.44)$ and were fairly confident in being able to apply sport psychology concepts and principles $(M=4.91, S D=1.02)$. On items addressing sport psychology expectations, coaches slightly disagreed that the benefits of SP were difficult to prove $(M=2.74, S D=1.41)$ and moderately disagreed that using SP services would make their athletes think more $(M=2.36, S D=1.20)$. Baseball coaches moderately disagreed that it would be difficult to trust an SPP $(M=2.0, S D=1.07)$ and that only athlete's with problems should see an SPC $(M=1.95, S D=1.08)$, indicating coaches generally trusted SPPs and do not think athlete's need to be 'broken' to benefit from SP services. Finally, coaches moderately disagreed that sport psychology services are counter-productive $(M=1.73$, $S D=.97$ ), indicating that they believe mental skills can be developed through practice. (See Table 4 for descriptive results for all non-SPARC-2 items and correlations) 
AN EXPLORATORY INVESTIGATION OF BASEBALL COACHES’ ATTITUDES

\section{Attitudes Toward Sport Psychology Services Within Independent Variables}

Correlations were run to examine relationships between demographic variables and outcome variables to determine if covariate statistical analysis should be run in place of ANOVAs or MANOVAs. Independent variables failed to meet the moderate criteria of $r=0.4$ to be considered covariates; therefore MANOVAs and ANOVAs were utilized. Unless otherwise specified, the level of probability to determine significance was set at .05 for all statistical analyses. Compared to previous studies using the SPA-RC, data from this study revealed similar internal consistency reliability indices evidenced in the following Cronbach's alpha estimates: Stigma Tolerance (.83), Confidence in SP Consultation (.81), Personal Openness (.65), and Cultural Preference (.83). Correlation coefficients between dependent variables and can be found in Table 6 .

Analysis of variance was completed to determine the relationship between participants' age and the dependent variables. Each of the following analysis yielded a small to moderate effect size. A one-way ANOVA revealed significant differences between participant's age and stigma tolerance $\left[\mathrm{F}(5,1,375)=2.75, \mathrm{p}<.05, \eta^{2}=.01\right]$, confidence in $\operatorname{SP}[\mathrm{F}(5,1,375)=3.27, \mathrm{p}<$ $\left..01, \eta^{2}=.01\right]$, personal openness $\left[F(5,1,375)=13.85, \mathrm{p}<.001, \eta^{2}=.05\right]$, and cultural preference $\left[\mathrm{F}(5,1,375)=4.02, \mathrm{p}<.001, \eta^{2}=.01\right]$. For stigma tolerance, a Tukey HSD test of multiple comparisons revealed that coaches over the age of 66 were significantly more likely to have positive attitudes towards SP than coaches 18-25. For confidence in SP, a Tukey HSD test of multiple comparisons revealed that coaches $36-45$ were significantly more likely to have confidence in SP than coaches 18-25. For personal openness, a Tukey HSD test of multiple comparisons revealed coaches 18 -25 were significantly less likely to be open to SP consultation than coaches 26 and older. Finally, a Tukey HSD test of multiple comparisons revealed that 


\section{AN EXPLORATORY INVESTIGATION OF BASEBALL COACHES’ ATTITUDES}

coaches $18-25$ were significantly more likely to have a strong cultural preference than coaches age 36-65.

Analysis of variance was completed to determine the relationship between years coaching and the dependent variables. A one-way ANOVA revealed significant differences between years coaching and stigma tolerance $\left[F(5,1,395)=3.75, p<.05, \eta^{2}=.01\right]$ and personal openness $[F$ $\left.(5,1,395)=2.27, \mathrm{p}<.001, \eta^{2}=.03\right]$. A Tukey HSD test of multiple comparisons revealed participants who had been coaching 0-5 years were significantly more likely to have negative attitudes towards SP than those coaching 6-10 and 26 or more years. Additionally, post-hoc analysis results revealed that participants coaching 0-5 years were significantly less open to SP consultation than those coaching 11 or more years.

Analysis of variance was completed to determine the relationship between education level and the dependent variables. A one-way ANOVA revealed significant differences between education level and stigma tolerance $\left[F(3,1,365)=3.63, p<.05, \eta^{2}=.01\right]$. A Tukey HSD test of multiple comparisons reveal that coaches who have earned master's degrees were significantly more likely to have positive attitudes towards SP than coaches with only bachelor's degrees. A one-way ANOVA also revealed a significant difference between primary job responsibility and personal openness $\left[\mathrm{F}(7,1,351)=2.78, \mathrm{p}<.01, \eta^{2}=.01\right]$. Post-hoc analysis revealed that head coaches were significantly more likely to be open to SP consultation than assistant coaches ( $p<$ $.05)$.

\section{Research Questions}

Hypothesis one, which stated, no significant relationships will be observed between a coach's age, years of coaching, education level, skill level coached, access to sport psychology services, or previous experience with sport psychology and use of sport psychology principles 


\section{AN EXPLORATORY INVESTIGATION OF BASEBALL COACHES’ ATTITUDES}

and services was analyzed using descriptive statistics (e.g., means, standard deviations, etc.), Chi-square test for Independence, and t-tests. Results for descriptive analysis of the independent variables can be seen in Table 1, Table $3 \mathrm{a}$ and Table $3 \mathrm{~b}$. A 2 X 2 chi-square test of independence was performed to examine the relationship between a coach's previous use of SP services as an athlete and current use of SP services. There was a statistically significant association between a coach's use of SP services during his athletic career and whether or not he currently utilized SP services, $\chi^{2}(1, N=1281)=34.53, \mathrm{p}<.001, r^{2}=.16$. Based on the odds ratio, this seems to represent the fact that the odds of coaches currently using SP services were 2.3 times more if coaches had previously used SP services as an athlete. A 2 X 2 chi-square test of independence was also performed to examine the relation between a coach's previous experience with SP services as an athlete or coach and intention to use SP services. There was a significant association between a coach's previous experience with SP services and intent to use SP services, $\chi^{2}(1, N=1300)=37.01, \mathrm{p}<.001, r^{2}=.17$. Each of the previous analyses yielded small effect sizes between variables.

Independent-samples t-tests were conducted to compare mean scores on all SPARC-2 subscales (i.e., stigma tolerance, confidence in SP, personal openness, and cultural preference) between groups on the following three survey items: "I am not currently using SP services but intend to in the next 12 months", "I am currently using SP services and intend to in the next 12 months", and "I have previous experience with sport psychology services as an athlete or coach". T-tests revealed significant differences on all four subscales between coaches who intend to use SP services and do not intend to use SP services sometime in the next 12 months. Similarly, independent samples t-tests revealed significant differences on all four subscales between 


\section{AN EXPLORATORY INVESTIGATION OF BASEBALL COACHES’ ATTITUDES}

coaches who have previous experience with SP services as a player or coach and those who have no previous experience with sport psychology. (See Table 5 for more information)

Hypothesis two, which stated, no significant relationships would be observed between a coach's stigma tolerance, confidence in SP, personal openness, and cultural preference and a coach's intention to use sport psychology services was analyzed using a one-way multivariate analysis of variance (MANOVA). Preliminary assumption testing was conducted to check for normality, linearity, univariate and multivariate outliers, homogeneity of variance-covariance matrices, and multi-collinearity. No serious assumption violations were noted. A one-way between-groups MANOVA was performed to investigate differences between coaches who intended to use sport psychology services and coaches who did not intend to use sport psychology services. Four dependent variables were used: (a) stigma tolerance, (b) confidence in SP, (c) openness, and (d) cultural preference. There was a statistically significant multivariate main effect for coaches who intended to use sport psychology services and who did not intend to use sport psychology services on the combined dependent variables: $\mathrm{F}(4,1295)=68.59, p=<$ .001 ; Wilks' Lambda $=.83$; partial eta squared $=.18$. Results indicated $18 \%$ of the variance in attitudes was explained by coaches' current use of sport psychology services. When the results for the dependent variables were considered separately for univariate interaction effects, all four variables reached statistical significance: (a) Stigma Tolerance: $\mathrm{F}(1,1298)=97.55, p=<.001$, partial eta squared $=.07$; (b) Confidence in SP: $\mathrm{F}(1,1298)=229.43, p=<.001$, partial eta squared $=.15 ;(\mathrm{c})$ Personal Openness: $\mathrm{F}(1,1298)=40.53, p=<.001$, partial eta squared $=.03$; and (d) Cultural Preferences: $F(1,1298)=7.05, p=<.01$, partial eta squared $=.005$. To reduce the Type 1 error rate (i.e., finding a significant result when there is not one), a Bonferroni adjusted alpha level of .0125 ( $\alpha / 4)$ was applied when examining mean scores (Pallant, 2005). An 


\section{AN EXPLORATORY INVESTIGATION OF BASEBALL COACHES’ ATTITUDES}

inspection of mean scores indicated that coaches who currently use SP services have a lower stigma tolerance, more confidence in SP services, are more willing to involved in SP consultation and mental training, and have a lower cultural identification than coaches not currently using SP services. Each of the previous analyses yielded small to moderate effect sizes.

Hypothesis three, which stated, no significant differences would be observed between skill level coached and stigma tolerance and openness to using sport psychology services, was analyzed using a MANOVA. Due to the design of the survey, differences between skill level coached and stigma tolerance and openness to using sport psychology services was not determined. In order to obtain the best representation of participants, coaches were asked to identify every skill-level they coached. A number of coaches said they worked with athletes across skill levels (e.g., high school and college etc.). Therefore, the researchers were unable to differentiate the primary skill level of athlete coached for each participant. Because of this flaw in the survey design, coaches could not be grouped by "skill level coached".

Hypothesis four, which stated, no significant differences would be observed between a coach's previous experience with sport psychology (i.e., as an athlete or coach) and a coach's stigma tolerance, confidence in SP, personal openness, and cultural preference, was also analyzed using MANOVA. For the purposes of this study, "previous experience with sport psychology" included any current or former experiences with SP services as a coach and/or athlete. Preliminary assumption testing was conducted to check for normality, linearity, univariate and multivariate outliers, homogeneity of variance-covariance matrices, and multicollinearity, with no serious violations noted. 
AN EXPLORATORY INVESTIGATION OF BASEBALL COACHES’ ATTITUDES

A one-way between-groups MANOVA was performed to investigate differences between coaches' previous experience with sport psychology and coaches' attitudes towards sport psychology. Four dependent variables were used: (a) stigma tolerance, (b) confidence in SP, (c) openness, and (d) cultural preference. There was a statistically significant multivariate main effect for coaches who had previous experience with sport psychology and coaches who did not have previous experience on the combined dependent variables: $\mathrm{F}(4,1396)=11.32, p=<.001$; Wilks' Lambda $=.97$; partial eta squared $=.03$. Results indicated three percent of the variance in attitudes was explained by coaches' previous experience with sport psychology. When the results for the dependent variables were considered separately for univariate interaction effects, all four variables reached statistical significance: (a) Stigma Tolerance: $\mathrm{F}(1,1399)=25.39, p=<$ .001 , partial eta squared $=.018 ;(b)$ Confidence in SP: $\mathrm{F}(1,1399)=26.77 p=<.001$, partial eta squared $=.019 ;(\mathrm{c})$ Personal Openness: $\mathrm{F}(1,1399)=6.78, p=<.01$, partial eta squared $=.005$; and (d) Cultural Preferences: $\mathrm{F}(1,1399)=11.91, p=<.01$, partial eta squared $=.008$. Each of the previous analyses yielded small to moderate effect sizes. To reduce the Type 1 error rate (i.e., finding a significant result when there is not one), a Bonferroni adjusted alpha level of .0125 $(\alpha / 4)$ was applied when examining mean scores (Pallant, 2005). An inspection of mean scores indicated that coaches who had previous experience with SP services as an athlete or coach reported a lower stigma tolerance, more confidence in SP services, were more open to SP consultation and mental training, and reported a lower cultural identification than coaches not currently using SP services. (See Table 3b for Means and Standard Deviations)

\section{Logistic Regression}

Due to the exploratory nature of the study, logistic regression was chosen over a stepwise analysis. Logistic regression was chosen over discriminant function analysis to simultaneously 


\section{AN EXPLORATORY INVESTIGATION OF BASEBALL COACHES' ATTITUDES}

evaluate the effects of seven predictor variables on the outcome variable. Assumptions were tested by examining normal probability plots of residuals and scatter diagrams of residuals versus predicted residuals. No violations of normality, linearity, or homoscedasticity of residuals were detected.

Descriptive results indicated $89.5 \%$ of participants intended to use SP services sometime in the next 12 months. Due to the exploratory nature of the study, limited research utilizing the SPARC-2, and no previous research focused specifically on baseball coaches, standard multiple logistic regression analysis was performed using SPSS REGRESSION to predict the probability that a participant would intend to use SP services sometime in the next 12 months (Field, 2009). The seven predictor variables were: (a) previous SP experiences as a player or coach, (b) years coaching, (c) education level, (d) stigma tolerance, (e) confidence in SP services, (f) personal openness, and (g) cultural preference. Continuous predictor variables (i.e., SPARC-2 subscale total scores) were recoded into four quartiles to more easily explain the differences within each subscale. When interpreting the results, each quartile should only compared against other quartiles within the subscale. See Table 7 and Table 8 for complete regression analysis.

A test of the full model versus a model with intercept only was statistically significant, $R^{2}=.32, \chi^{2}(19, N=1300)=218.31, \mathrm{p}<.001$. The Cox and Snell R Square and the Nagelkerke $\mathrm{R}$ Square values from the logistic regression analysis revealed the amount of variation in the dependent variable explained by the model. Results suggested that between $15.7 \%$ and $32.2 \%$ of the variance could be explained by the predictor variables. The model was able to correctly classify $98.9 \%$ of participants who intend to use SP services sometime in the next 12 months and $15.8 \%$ of those who do not, for an overall success rate of $90.2 \%$. This was slight improvement from the initial model, which had an overall success rate of $89.6 \%$. Table 7 shows the 


\section{AN EXPLORATORY INVESTIGATION OF BASEBALL COACHES' ATTITUDES}

percentage of participants on each independent variable who intend or do not intend to use sport psychology services within the next calendar year. Table 8 shows the adjusted odds ratio, confidence intervals, and significance level for each of the predictors. Results of the previous analyses yielded a medium effect size.

Employing a .05 criterion of statistical significance, the following predictor variables revealed a statistically significant partial effect on coaches' reported intent to use sport psychology services: (a) previous SP experience as a player or coach, (b) stigma tolerance, (c) confidence in SP consultation, and (d) personal openness. Specifically, the odds ratio for previous SP experience indicated that when holding the other variables constant, participants who had previous SP services as an athlete or coach were 2.91 times as likely to intend to use SP services as coaches with no previous experience with sport psychology services. The odds ratio

for stigma tolerance indicated that participants with the most negative attitudes (i.e., $4^{\text {th }}$ quartile) towards sport psychology were .30 times less likely to intend to use sport psychology services than those with the lowest stigma tolerance scores. The odds ratio for confidence in SP consultation indicated that participants with the highest confidence level (i.e., $4^{\text {th }}$ quartile) were 36.6 times as likely to intend to use SP services than those with the lowest confidence in SP services (i.e., $1^{\text {st }}$ quartile). Logistic regression results indicated that the variables 'years coaching' and 'education level' were not significant predictors of a coach's intention to use sport psychology services. (See Table7 and Table 8) 
AN EXPLORATORY INVESTIGATION OF BASEBALL COACHES’ ATTITUDES

\section{CHAPTER V}

\section{Discussion}

While some research has examined athletes' attitudes and experiences with sport psychology (e.g., Martin, 2005), limited research has examined coaches' perceptions and experiences with sport psychology (Zakrajsek \& Zizzi, 2007). Furthermore, this line of research has been narrow in scope, examining only a few coaches' views from select sports (i.e., track and field, swimming, and football) (Zakrajsek \& Zizzi, 2007; 2011). The literature also seems to be lacking insight into coaches' perceptions from one particular sport across all age groups and competition levels. Coaches' relationships with sport psychology could be valuable because some previous research indicates coaches' general philosophies, opinions, and attitudes may impact their player's views, opinions, and development (e.g., Dieffenbach, Gould, \& Moffett, 2002).

The purpose of the current study was to survey American College Baseball Association (ABCA) members' and National Collegiate Athletic Association (NCAA) head baseball coaches' attitudes and possible experiences with sport psychology. More specifically, the current study examined baseball coaches': (a) stigma tolerance towards sport psychology, (b) confidence in sport psychology services, (c) openness to hiring a sport psychology professional, (d) cultural preference of sport psychology professionals, (e) knowledge of sport psychology principles and concepts, and (f) current or previous experiences with sport psychology. The following sections will provide a discussion of the results reported above, possible meaning of the results of this study, the results' link to previous research, and the implications of the findings. Lastly, limitations of the study and recommendations for future research will be provided. 
AN EXPLORATORY INVESTIGATION OF BASEBALL COACHES' ATTITUDES

\section{General Demographic Information}

In the current study, approximately 7,800 emails were sent to ABCA members and NCAA head baseball coaches, over 1,700 coaches responded to the initial request for participation, and 1,401 surveys were used in the final analysis. This response rate was greater than previous studies examining coaches' attitudes towards sport psychology (e.g., Wrisberg, Loberg, Simpson, Withycombe, \& Reed, 2010), and appears to be the largest sample to date examining coaches and sport psychology. The $23 \%$ response rate appears to be comparatively higher than average for research utilizing email survey methodology (Dillman, 2007). The somewhat above-average response rate may reflect the importance that baseball coaches place on the psychological aspects of the game. It may however, merely reflect general curiosity or interest that baseball coaches have in this area of performance. Since coaches are usually in charge of providing their players with access to sport psychology resources and services (Partington \& Orlick, 1987), these results are encouraging.

In exchange for participation, respondents could choose to receive additional "coachspecific" sport psychology information and request a free copy of the survey results with information about how their peers view sport psychology. Of the 1,700 respondents, 650 requested the above option. Despite the offer to receive a free copy of the results, less than $40 \%$ of respondents requested such action, perhaps reflecting coaches' general willingness to contribute to the growing the awareness, knowledge, and understanding of baseball coaches' relationship with sport psychology. On the contrary, coaches may not be interested in knowing what other coaches think about sport psychology.

The majority of respondents in the current study said they were college-educated, Caucasian, and male. While it is unclear how the sample population compares to all coaches 


\section{AN EXPLORATORY INVESTIGATION OF BASEBALL COACHES’ ATTITUDES}

asked to participate, it seems possible that baseball coaches across the country may resemble this demographic profile. This finding may also reflect the type of baseball coach who selected into participating in the study. Although previous research has demonstrated large gender differences between men and women with regards to help-seeking behavior, openness to psychological services, and general acceptance and understanding for psychology (e.g., Constantine \& Gainor, 2004; Good, 1995), it would be impossible to determine differences in the current study since close to $100 \%$ of participants in the current study were male. Coaching jobs at the high school, collegiate, and professional level usually require at least bachelor's degree; it would also make sense that the majority of participants have graduated from college. However, considering there are over 11 million baseball players across the country representing a wide variety of ethnicities, nationalities, and cultures, is it somewhat alarming to find out that the overwhelming majority of baseball coaches are Caucasian. According to a 2011 study, minorities made up close to $40 \%$ of Major League Baseball players and $42 \%$ of all professional baseball coaches (Lapchick \& Harrison, 2011). These results of the current study revealed a discrepancy between the ethnic and cultural proportion of baseball players to baseball coaches. This could speak to the underrepresentation of other ethnicities, nationalities, or cultures in the coaching profession.

Participants in the current study said that it was very important for their athletes to have strong mental skills, and generally believed that those skills can be developed via mental skills training. Previous research similarly suggests that coaches consider the "mental game" of baseball to be an important component of the team and individual performance (Gmelch, 2000). Coaches' in the current study also believed that approximately $70 \%$ of baseball performance was related to psychological factors. Even though this is the first comprehensive assessment of baseball coaches, these percentages approximate previous research on collegiate and professional 


\section{AN EXPLORATORY INVESTIGATION OF BASEBALL COACHES’ ATTITUDES}

baseball players, who reportedly believed that roughly $80 \%$ of baseball performance could be psychological (Hanson \& Ravizza, 2003). More than 98\% of respondents reported having a previous athletic career, so it would make sense that participants placed so much emphasis on the mental side of the game.

In contrast to the level of importance that coaches' placed on psychological skills, respondents reported that they only dedicate approximately one-quarter of practice time to this area of the game. The discrepancy between importance of psychological factors and time spent developing mental skills is consistent with some previous research that found coaches devoted little time to developing mental skills (e.g., Harris \& Harris, 1984). These results are somewhat surprising, because numerous sport psychology researchers have suggested psychological skills training may positively impact athletic performance (e.g., Gould et al., 1990). The difference in reported "perceived" importance and "actual" time investment in mental skills development provides sport psychology professionals (SPPs) with helpful information to help meet the needs of baseball coaches and players in reduced amounts of time.

There are several possible reasons why many coaches seem to place considerable emphasis on the mental game, but likely dedicate comparatively little practice time to developing the mental skills of their athletes. Perhaps some coaches do not know how to effectively implement sport psychology principles during practice. On the other hand, Zakrajsek and Zizzi (2007) discussed the fact that despite the wide array of the sport psychology books, videos, or workshops available to coaches, coaches may not know how to access sport psychology services for their team or how to use SPPs most effectively. Coaches may assume that players have the ability to develop these skills without additional help; or are convinced that players with the strongest mental skills will separate themselves by out-performing their teammates on the field, 


\section{AN EXPLORATORY INVESTIGATION OF BASEBALL COACHES’ ATTITUDES}

consequently weeding out players who are not "mentally tough" enough to consistently compete at higher levels. Therefore, coaches may be less likely to devote practice time to developing mental skills, allowing for the "natural selection" of players with the best physical and mental skills to emerge as the season progresses. In a sport like baseball where there is such a big difference between the number of players on any given team and the number of players needed to play a game, this approach may be appropriate. Finally, other 'competing interests' or lack of resources may play a role in the discrepancy between how important coaches might perceive psychological skills to be and how much time is seen as available, and therefore spent focusing on developing mental skills during practice. Perceived and barriers such as time restrictions, practice rules limiting player access and other regulations set by governing bodies (e.g., the NCAA), and costs associated with consultation may impact the extent to which baseball coaches across levels invest time and effort on psychological skills training. Consequently, these and other barriers can negatively impact the 'value-added' services programs are willing to invest in. With college and high school budgets decreasing, coaches may decide that their money would be better spent buying their players better equipment or new uniforms, leaving little money to hire an SPP. Collegiate and high school coaches may decide to invest their resources into other necessary services such as athletic trainers, strength and conditioning coaches, or sport nutritionists.

\section{Sport Psychology Attitudes (SPARC-2)}

Coaches in the current study generally said that they owned a positive view of sport psychology. On average, participants reported positive attitudes toward sport psychology services, were fairly confident in sport psychology consultation, were relatively open to seeking out sport psychology services, and did not have a strong cultural preference towards SPPs. 


\section{AN EXPLORATORY INVESTIGATION OF BASEBALL COACHES’ ATTITUDES}

Similarly, results from a previous study (Zakrajsek \& Zizzi, 2007) suggested all subscales except cultural preference were factors that contributed to coaches' attitudes towards sport psychology. Interestingly, these findings were contrary to previous research on preferences in general helpseeking professionals (e.g., Atkinson \& Lowe, 1995; Martin, 2005), which could indicate cultural preference may only be a factor in more traditional (i.e., non-athletic) counseling contexts. There were several participants who reported negative attitudes towards SPPs and SP services, however the general consensus was that participants had positive attitudes towards sport psychology and appreciated the information and expertise SPPs can offer their athletes and teams.

Results of the current study indicated that previous experience working with a sport psychology professional may positively impact coaches' attitudes towards SP. Coaches who had previous experience with sport psychology services as an athlete or coach showed a significant difference across all four SPARC-2 subscales than coaches who have no experience with sport psychology services. Additionally, results of the current study indicated a significant difference across all four SPARC-2 subscales between coaches who currently use SP services and those who do not. Results revealed significant differences in stigma tolerance, confidence in sport psychology services, openness to hiring a sport psychology professional, and cultural preference between coaches who intend to use sport psychology services and those who do not (See Table 5). Regardless of age, education level, coaching experience, or any other demographic characteristic, coaches in the current study reported that experience with SP services significantly increased positive attitudes towards SP. These findings are similar to previous research using the SPA-RC on coaches' attitudes towards sport psychology, which examined swimming and track and football (Zakrajsek \& Zizzi, 2007; Zakrajsek, et al., 2011, respectively) 


\section{AN EXPLORATORY INVESTIGATION OF BASEBALL COACHES’ ATTITUDES}

coaches, respectively. Results from both studies suggested previous experience with sport psychology is the most consistent factor influencing coaches' attitudes (Martin, Zakrajsek, \& Wrisberg, 2012). Coaches with more sport psychology knowledge and previous experience with sport psychology or counseling were more confident and open to utilizing services, had more positive attitudes toward sport psychology, and had more realistic expectations compared to coaches with inadequate knowledge and no prior experience (Zakrajsek \& Zizzi; Zakrajsek, et al., 2011). Some early research in this area showed that some coaches believed sport psychology could help promote athletic development (Silva, 1984).

With regard to confidence in sport psychology, both Anderson et al. (2004) and Martin (2005) found that participants who had previous experience with sport psychology demonstrated more confidence in seeking out sport psychology services. In another study of 50 NCAA Division I-III head coaches representing 20 sports, Walker and Eslinger (2003) observed that coaches they studied had positive opinions towards sport psychology.

With regard to stigma tolerance, Martin (1997) found that level of stigma tolerance was related to whether or not a coach utilizes an SPP. As far as openness to sport psychology consultation, previous research found that participants were more open to utilizing SP services if they had previous experience with sport psychology (Wrisberg et al., 2009). Similarly, Sullivan and Hodge (1991) reported that over $90 \%$ of coaches indicated an interest in sport psychology and were open to implementing sport psychology services with their teams.

Despite the similarities across all three studies that used the SPA-RC, there were some differences in coaches' responses based on the type of sport (e.g., non-contact, masculine) that was investigated. Specifically, results suggested coaches from individual sports that were not masculine oriented or dominated by males (i.e., swimming and track) reported lower stigma, 


\section{AN EXPLORATORY INVESTIGATION OF BASEBALL COACHES’ ATTITUDES}

higher confidence, and less openness to sport psychology services than coaches from masculine, male-dominated sports (i.e., Texas high school football) (Zakrajsek et al, 2011; Zakrajsek \& Zizzi, 2007). Masculine oriented sport coaches also reported a greater preference for working with an SPP with similar background to themselves and their athletes. Coaches from the current study reported attitudes more similar to coaches that are from non-contact sports with equal gender representation. These results are remarkable considering baseball is a male-dominated and masculine oriented individual sport played in a team context. These results may suggest baseball is a hybrid sport in the sense that it is a team sport dominated by males, yet coaches' attitudes towards sport psychology reflect those more gender-neutral and individual sports. Results and the implications of these findings should be further explored.

Taken together, findings from the current study and the literature suggest that coaches believe exposure to sport psychology often has a positive impact on the attitudes and confidence levels of athletes and coaches toward sport psychology. Exposure and positive experiences may also foster decreased stigma and intolerance. This evidence may encourage SPPs to seek out opportunities where they can speak with coaches about how sport psychology may help improve the performance and functioning of their athletes and teams.

Coaches who participated in this study said that they generally value sport psychology and were familiar with sport psychology principles and concepts. Some research on attitudes towards sport psychology has indicated that high school athletes prefer approval from their coach when utilizing sport psychology services (Blom, Hardy, Burke, \& Joyner, 2003). It appears that athletes want to know their coaches value the range of services SPPs can provide and perhaps the use of such help. This seems important because the attitudes and familiarity with sport psychology could also impact their athletes, who may subsequently be more inclined to use sport 


\section{AN EXPLORATORY INVESTIGATION OF BASEBALL COACHES’ ATTITUDES}

psychology services and also become the next generation of coaches. Therefore, it may be helpful to encourage coaches to discuss their attitudes and experiences with sport psychology with their athletes. This would demonstrate that sport psychology is a useful and important topic of discussion, and could encourage athletes' to be more proactive in seeking out sport psychology services.

\section{Dependent Variables}

Despite coaches' generally positive attitudes towards sport psychology, there were some significant differences between coaches separated by groupings on the dependent variables. Results of the current study revealed participants' attitudes towards sport psychology varied based on their age, coaching experience, highest education level, and primary job responsibility. Specifically, coaches younger than 25 said they viewed sport psychology significantly more negatively than coaches older than 66, had significantly lower confidence in SP than coaches 3645, were more likely to have a strong cultural preference than coaches older than 35 , and were significantly less likely to be open to sport psychology than all other coaches. These findings mirror some previous studies on coaching age and experience regarding sport psychology (Zakrajsek \& Zizzi, 2007; Walker \& Eslinger, 2003). Previous research has indicated that younger and less educated men maybe be less likely to engage in help-seeking behaviors than men who are older or have a higher level of education (Berger, Levant, McMillan, Kelleher, \& Sellers, 2005; Parslow \& Jorm, 2000). Similarly, other researchers have found people generally become more accepting of psychological help as they get older (Esters, Cooker, \& Ittenbach, 1998; Garland \& Zigler, 1994), and older coaches are more apt to use sport psychology services than younger coaches (Walker \& Eslinger, 2003). 


\section{AN EXPLORATORY INVESTIGATION OF BASEBALL COACHES’ ATTITUDES}

Much of the previous limited research on coaches' attitudes towards sport psychology has examined experienced elite and professional level coaches (Zakrajsek, et al., 2011). Results of the current study indicated coaches with less than five years of experience had significantly more negative attitudes towards sport psychology and were less open to hiring an SPP than their more experienced peers. Similarly, Zakrajsek, Martin, and Zizzi (2011) found that high school football coaches with six or fewer years of coaching experience were significantly less willing to utilize sport psychology services than more experienced coaches. Considering that younger coaches have grown up in an era where sport psychology has become a more widely discussed topic among coaches and players across sports (Williams \& Straub, 2006), it is somewhat surprising that younger coaches possessed more negative attitudes towards sport psychology and were less inclined to hire sport psychology professionals to work with their athletes or teams.

There are several possibilities why younger coaches said they were less accepting of sport psychology than their experienced peers. For example, inexperienced coaches may be more concerned with learning the strategies and techniques of successful coaching than concerning themselves with the psychological components of the game. Perhaps younger coaches felt the need to become competent in the physical and tactical areas of baseball before focusing their effort on understanding how mental skills impact performance. In addition to spending time learning baseball strategy, novice coaches may not know how to assess their athlete's mental skills early in their coaching career. Since athletes' level of physical skills (e.g., strength and speed) are often tangible and more visible to the eye than mental skills (e.g., controlling arousal levels), novice coaches may naturally spend their time learning how to assess and evaluate players and situations based on physical skills. It is possible that coaches only feel competent enough to focus their energy on learning about the psychological aspects of 


\section{AN EXPLORATORY INVESTIGATION OF BASEBALL COACHES' ATTITUDES}

performance after they become confident in their abilities to properly assess physical skills, teach proper strategies and techniques, and become experts in each of the competencies coaches need to develop to become successful.

Regarding participant education level, results indicated coaches with graduate degrees had more positive attitudes towards sport psychology than those who have only earned undergraduate degrees. This may reflect the fact that graduate degrees are often completed in the field in which one intends to work. For example, coaches in the current study with graduate degrees may have received them in majors such as kinesiology, exercise science, or even sport psychology. Therefore, their education may provide training and experience in content areas such as mental training and psychological skill development. However, there is no guarantee that respondents were trained in kinesiology, coaching, sport sciences, or any other related discipline. Participants were not asked to provide their undergraduate or graduate degree major or area of specialization. These results could signify that a based on any number of factors, postgraduate experience may lend itself to more positive attitudes towards help-seeking behavior. This information may be helpful in designing future studies examining coaches' attitudes toward sport psychology.

With regards to primary job responsibility, head coaches said that they were significantly more open to hiring SPPs than assistant coaches. This finding was somewhat unanticipated, since assistant coaches' opinions and beliefs often mirror the philosophies of their head coaches. While the design of the study does not provide insight into whether any two or more participants are on the same coaching staff, the discrepancy may indicate the difference in thought processes between head and assistant coaches. Head coaches were perhaps more willing to take a chance on hiring an SPP, whereas assistant coaches may not be comfortable enough in their role to 


\section{AN EXPLORATORY INVESTIGATION OF BASEBALL COACHES’ ATTITUDES}

suggest to their head coach that their athletes could benefit from sport psychology services.

Further, it does not appear that responding head coaches and assistant coaches differed on stigma tolerance of sport psychology. Therefore, the differences in openness to SP consultation may have reflected assistant coaches' unwillingness to challenge the approach of their head coach.

There were no additional statistically significant differences observed between coaches across SPARC-2 subscales with regard to their primary job responsibility. In retrospect, a better predictor of attitudes towards sport psychology may have been 'skill level coached'. Since there are 'head coaches', 'assistant coaches', and 'position coaches' across competition levels (e.g., professional, collegiate, high school, club/select), measuring attitudes by 'skill level coached' may have provided a clearer understanding of how coaches between different age groups perceive sport psychology. For example, it would have been interesting to compare the attitudes of coaches who primarily work with youth athletes to those who work only with collegiate athletes. Further research on attitudes towards sport psychology should examine the differences in coaches' across competition level.

\section{Sport Psychology Intentions}

Results from the logistic regression revealed that nearly $90 \%$ of respondents said that they intended to use sport psychology services in the next twelve months with proper financial resources. More specifically, over $95 \%$ of coaches who had previous experience with sport psychology services and $85 \%$ who had no experience with sport psychology intend to use sport psychology services in the future with proper financial resources. These findings suggested that baseball coaches who responded to the survey may be very interested in sport psychology. The specific type of services (e.g., attending workshops, hiring SPPs) coaches intend to use was not identified. However, it appears many baseball coaches today across levels of participation see 


\section{AN EXPLORATORY INVESTIGATION OF BASEBALL COACHES' ATTITUDES}

the value in sport psychology consultation. In a recent study on Texas high school football coaches, $48 \%$ had at least minimal intention of using sport psychology services within six months after participating in the study (Zakrajsek, et al., 2011). Specifically, researchers found confidence in sport psychology consultation accounted for approximately $30 \%$ and personal openness accounted for roughly $3 \%$ of the total variance in coaches' intentions to use sport psychology services.

The current study appears the first that examined baseball coaches' intentions to use sport psychology services. This discrepancy could reflect a difference in test taking conditions, the way the item was worded, or the fact that "pro sport psychology" coaches self-selected into the study. Nevertheless, baseball coaches' intentions to use sport psychology services appear to be significantly higher than coaches from other sports. Further investigation of 'intent to use SP services' should be completed to obtain a more accurate representation of coaches across sports and skill levels.

Regression analyses suggested that the following four variables significantly predicted intention to use sport psychology services: (a) previous sport psychology experience, (b) stigma tolerance, (c) confidence in sport psychology, (d) and personal openness. Specifically, coaches who had previous experience with sport psychology services were approximately three times as likely to intend to use sport psychology services in the future compared with coaches who had never utilized sport psychology services. Also, coaches who had the highest confidence in sport psychology services, those with the lowest stigma tolerance, and coaches open to hiring a sport psychology professional were more likely to intend to use sport psychology services than coaches with less confidence and more stigma tolerance toward sport psychology. Results from the current study suggested cultural preference and coaches' education level did not significantly 


\section{AN EXPLORATORY INVESTIGATION OF BASEBALL COACHES’ ATTITUDES}

predict intentions to utilize sport psychology services. These results are similar to some previous studies examining sport psychology attitudes and intentions. For example, Anderson et al. (2004) found that athletes' confidence in sport psychology consulting was a significant predictor of intentions to use sport psychology services. Also, in the first study utilizing the SPA-RC to assess swimming and track coaches attitudes and intentions to use sport psychology services, Zakrajsek and Zizzi (2007) found that confidence in sport psychology, expectations of sport psychology services, and stigma tolerance were all significantly related to intentions to use sport psychology services. More specifically, a stepwise multiple regression analysis provided support for an exploratory model for the following three factor solution that accounted for $38 \%$ of the total variance in intentions to use SP services: confidence in SP (34\% of variance), stigma tolerance $(3 \%)$, and expectations of the SP consultation (1\%). Results of previous study (Zakrajsek \& Zizzi, 2007) revealed that personal openness and previous exposure did not significantly predict coaches' intentions.

Results from the current study could be useful to coaches, athletes, and SPPs for a number of reasons. For baseball coaches, it appears that experiences with sport psychology services or professionals are generally positive, resulting in a lower stigma tolerance (i.e., positive attitude) towards SP, higher perceived confidence in SP services, and greater openness to utilizing the services of a sport psychology professional. In other words, exposure to sport psychology information or professionals may impact the factors that may help coaches decide whether to use sport psychology services in the future. Therefore, it appears SPPs could benefit from engaging in activities that facilitate direct exposure to baseball coaches. For example, SPPs should look for opportunities to speak at coaching conferences, provide coaches' workshops to 


\section{AN EXPLORATORY INVESTIGATION OF BASEBALL COACHES' ATTITUDES}

high school or collegiate leagues, or contact their local coaching organizations to offer their services.

\section{Sport Psychology Experiences}

In addition to evaluating baseball coaches' attitudes towards sport psychology, an attempt was made to better understand coaches' previous experience with SP services. It appeared that a significantly higher number of participants from this study had experience with sport psychology than previous studies. Results of the current study indicated over $40 \%$ of participants had previous experience with sport psychology services and over 30\% of coaches reported currently using SP services with their athletes and teams. Analysis revealed coaches were also significantly more likely to utilize sport psychology services with their current teams if they had some previous experience with sport psychology during their athletic or coaching career.

Before assuming their current role, many coaches previously competed in their sport as athletes, so any attitudes, perceptions, or experiences they previously had with sport psychology may influence their current attitudes and relationship with sport psychology. While this study examined only baseball coaches, some previous research on athletes' and coaches' experiences with sport psychology suggests (Zakrajsek \& Zizzi, 2007) revealed that despite the fact that $87 \%$ of participants had previous SP experience, only $22 \%$ of the coaches surveyed were currently working with a sport psychology professional. In another study on high school football coaches, researchers found that less than five percent of participants had previously used SP services (Zakrajsek, et al., 2011). While it is worth noting that equal comparisons should not be made between results of the current study and those from a homogenous sample such as Texas high school football coaches, the discrepancy could shed light on the unique differences between 


\section{AN EXPLORATORY INVESTIGATION OF BASEBALL COACHES’ ATTITUDES}

coaches across sports, geographical locations, and competition levels. This contrast in results could reflect attitudes unique to baseball coaches.

\section{Non-SPARC-2 Survey Items}

In addition to items examining coaches' attitudes and experiences with sport psychology, participants in the current study answered several items that addressed their expectations of sport psychology services and questions about how they believe other baseball coaches perceive sport psychology (i.e., subjective norms) (See Table 4). Each item was unique to this study and had not been tested or validated in previous research, therefore only descriptive statistical analysis could be interpreted. Regardless of these limitations, responses on such items provided important insight into how coaches value sport psychology services, revealed information about how baseball coaches perceived sport psychology, and could inform SPPs how to better communicate their services and skills to baseball coaches. The results are particularly interesting because they reflect the experiences and perceptions of only baseball coaches.

\section{Sport Psychology Knowledge and Education}

The most common ways for coaches to gain exposure and knowledge of sport psychology information includes attending organized clinics or presentations, taking academic coursework, or reading books on the subject (Zakrajsek \& Zizzi, 2007). In the current study, approximately $90 \%$ of participants said they were familiar with sport psychology in some way. Similarly, over $80 \%$ of participants reported having at least some sport psychology training in the form of a taking a college class, reading a book on sport psychology or mental training, or attending a conference workshop or presentation. Results from this study are relatively consistent with previous research, which indicated approximately $60-70 \%$ of coaches' previous exposure to sport psychology is in the forms of workshops, clinics, or classes (e.g., Hodge, 1991; 


\section{AN EXPLORATORY INVESTIGATION OF BASEBALL COACHES’ ATTITUDES}

Rice, 1997). Researchers have found that attending a workshop on sport psychology resulted in improving coaches' awareness of the benefits of implementing SP services and increased coaches' interest level with regard to utilizing an SPP (Zakrajsek \& Zizzi, 2007). Gould, et al. (1990) also found that experiences such as psychological skills workshop can enhance interest and intended use of mental skills in the short term, but this intention decreases over time.

Considering the wide array of mental training resources available in the form of websites, e-books, magazine articles, mobile apps, and online materials, it is not surprising that such a high percentage of coaches from the sample population reported having at least some nominal exposure to sport psychology information. What is perhaps more remarkable is that coaches were confident in their ability to teach and apply sport psychology without the direct help of sport psychology professionals. In contrast to previous research, baseball coaches in this sample generally believed they have the knowledge base to implement sport psychology with their athletes and teams. More specifically, participants in the current study felt they generally knew enough about sport psychology to teach their athletes how to develop mental skills and are confident in their ability to implement sport psychology principles with their teams. These results are inconsistent with some previous research, which found coaches believed they lacked the education to effectively teach athletes psychological skills that can improve performance (Figone, 1999). Similarly, results from another distant study indicated that although over 65\% of coaches had previous exposure to sport psychology in the form of workshops or academic courses, they still did not feel they had an adequate knowledge base about sport psychology (Sullivan \& Hodge, 1991). These studies were completed over 15 years ago, so a lot may have changed in the minds of coaches over that time span. Nevertheless, along with the information obtained in the current study, these findings provide SPPs insight into some of the potential 


\section{AN EXPLORATORY INVESTIGATION OF BASEBALL COACHES’ ATTITUDES}

barriers facing practitioners; specifically that coaches feel like they can do the work on their own. Why would coaches hire a professional when they sense they are competent enough to do the work themselves?

What is it about sport psychology that makes many baseball coaches feel confident enough to try to implement sport psychology principles without adequate training or education? Conversely, what is it about baseball coaches that makes them feel that reading a book or attending a workshop is enough experience to effectively implement sport psychology with their teams? One could argue this approach is analogous to someone reading a textbook or going to a medical conference about a new surgical procedure and subsequently feeling confident enough to ask an attending doctor to scrub in for surgery. While clearly an over-exaggeration, this example does highlight the concept of 'practicing' a discipline possibly without proper training, education, or experience. Perhaps some coaches have a background in kinesiology or exercise science, disciplines where sport psychology concepts are often discussed and written about. Maybe coaches believe their background has provided them with the necessary training and knowledge base to administer sport psychology services themselves.

Despite the general confidence in integrating sport psychology with there teams, results suggested that younger coaches may be more confident than their more experiences peers. These results may reveal a generational shift; the younger generation of coaches may be overconfident or feels competent in their ability to integrate sporty psychology principles with their teams without proper training or education. Perhaps younger coaches simple “don't know what they don't know", and veteran coaches are more willing to acknowledge that they may not be equipped with the knowledge to effectively teach their athletes about the mental game. Admittedly, the consequences of wrongfully implementing sport psychology with one's team are 


\section{AN EXPLORATORY INVESTIGATION OF BASEBALL COACHES' ATTITUDES}

not likely life and death. However, there appears to be something innocuous enough about sport psychology where coaches ostensibly feel confident enough to "do" sport psychology with their teams without any formal training or education.

To date, researchers have yet to determine how coaches conceptualize sport psychology and how they apply sport psychology or mental training with their athletes and teams. Likewise, the factors that contribute to many baseball coaches' elevated confidence in their ability to implement sport psychology principles or concepts remains unclear. Interestingly, Zakrajsek and Zizzi (2007) found a poor correlation between previous exposure to sport psychology and realistic sport psychology expectations (e.g., confidentiality, role of SPP), suggesting SPPs should not assume that coaches with SP exposure or experience completely understand how to apply sport psychology with their athletes. Therefore, coaches may not well understand the process of consultation or have difficulty dealing with the ethical guidelines SPPs abide by. In addition to perceived competency in this area of performance, coaches may feel that it's easier to just 'eliminate the middle man' from the equation. Despite these concerns, results from the current study indicate that some baseball coaches may be comfortable enough to apply sport psychology principles without the help of an SPP.

On the other hand, perhaps participants feel confident in their ability to apply sport psychology with their teams because of a combination of perceived, sufficient education and coaching experience. Most of the participants in the current study said they had either previously worked with a sport psychology professional, had minimal education in mental training, or had attended at least one workshop on sport psychology. However, the participants in the current study were not asked to provide their definition of 'sport psychology' or explain how they apply SP principles with their athletes. 
Despite the obvious differences in sport psychology background between coaches and SPPs with regards to education, training, and being able to effectively communicate and apply SP principles, it also remains unclear how successful coaches are at relaying such information. For example, some research has shown that even when athletes attempt to improve psychological skills during competition, they may be doing so incorrectly (Frey, Laguna, \& Ravizza, 2003; Monserrat, 2004). Regardless of the number of workshops attended, books read, or videos watched, it may be difficult for coaches to effectively apply sport psychology theory into practice; they are trained baseball coaches, not sport psychology professionals. Without an SPP on staff, or at least without easy access to a sport psychology professional, coaches may have difficulty properly applying SP principles with their teams.

Without sufficient training or education, effectively implementing sport psychology with their teams may be difficult. For example, they may not be able to recognize if their methods are working or know the theoretical framework behind specific sport psychology approaches. Additionally, they may not know how to work with athlete on a certain performance concern (e.g., confidence, focus, stress management) and have trouble recognizing behaviors or signs that would indicate an athlete possibly has clinical issues (e.g., depression, anxiety). There are a number of additional concerns (e.g.. ethical, legal, etc.) to be considered if coaches are implementing sport psychology techniques or principles without proper training or professional help. However, the current study did not address how coaches are integrating sport psychology with their teams, so it is possible that their definition of the "mental game" is different from that of a trained SPP. Since it appears that many baseball coaches are already implementing sport psychology techniques with their teams, it would be useful for SPPs to contact their local coaching leagues or conferences and offer their services to these organizations. At minimum, 
AN EXPLORATORY INVESTIGATION OF BASEBALL COACHES’ ATTITUDES

coaches should have the contact information for the Association of Applied Sport Psychology Certified Consultants in their geographical region.

\section{Sport Psychology Expectations and Coaches Subjective Norms}

Research on baseball coaches has indicated coaches' attitudes towards sport psychology can directly impact players' views of mental training (Hanson \& Ravizza, 2003). Coaches with views towards sport psychology, both positive and negative, can trickle-down to their athletes, potentially having a lasting effect on athletic and personal development (Hanson \& Ravizza, 2003). With this in mind, results from respondents in current study on items related specifically to baseball coaches' sport psychology expectations and subjective norms are encouraging (See Table 4). Not only did participants report generally positive attitudes towards sport psychology, coaches did not appear to have much resistance towards the scope of work SPPs do with their athletes and are not necessarily concerned about keeping their work with SPPs a secret. More specifically, coaches generally disagreed that it would be difficult to trust an SPP working with their athletes, strongly disagreed that they would think less of their athletes if they worked with an SPP, generally did not believe working with an SPP s bad for an athlete's reputation, and generally disagreed that SPPs should only work with athletes who have issues or problems. These results are somewhat contrary to some previous research on athletes and coaches, which has indicated there may be a stigma associated with individuals who consult with an SPP (e.g., Linder et al., 1989; Martin et al., 2001). Coaches and athletes have been known to think sport psychology is only for "problem athletes" or "head cases" (Linder, 1989; Ravizza, 1988), and SPPs can sometime be associated with the term "shrink" (Ravizza, 1990). This finding could either reflect a selection bias of coaches who participated in the survey because they have a positive view of sport psychology, a more widespread acceptance of sport psychology across the 


\section{AN EXPLORATORY INVESTIGATION OF BASEBALL COACHES’ ATTITUDES}

baseball landscape, or a combination of the two. Nevertheless, these results are encouraging and may possibly indicate that some coaches no longer stigmatize or label athletes with problems or issues as "head cases", often failing to acknowledge that they may simply need some outside help that the coach is not trained to provide. It appears that many participants truly valued the skills and services SPPs can bring to their teams. However, it is highly unlikely that every baseball coach in the country has the same attitudes and perceptions towards sport psychology. Therefore, SPPs should remain cautiously optimistic when interpreting the results.

Coaches in the current study were also asked about their sport psychology expectations and perceptions of how other coaches view sport psychology. Results for coaches' subjective norms revealed coaches slightly agreed that they have access to sport psychology services, that coaches they respect utilized sport psychology services, and that other coaches on their staff would support hiring a sport psychology professional (See Table 4). Results also indicated that coaches slightly disagreed as to whether they would want work with an SPP to be kept a secret, and were not concerned if other coaches found out that their athletes or coaching staff was working with an SPP. These results suggest that some coaches are not concerned about their peers finding out that their teams or athletes are working with an SPP. The possible lack of concern is encouraging because it may signify a low stigma associated with sport psychology and SPPs, and sheds light on the fact that some coaches today are comfortable discussing sport psychology and their work with SPPs with other coaches. The more that coaches know that their peers value SPPs and utilize sport psychology services, the more likely they may be to do the same.

However, there seems to be a gap between coaches' reported utilization of sport psychology information and their awareness that other coaches utilizing sport psychology 


\section{AN EXPLORATORY INVESTIGATION OF BASEBALL COACHES’ ATTITUDES}

services. For example, close to forty percent of baseball coaches in this study said they were currently using sport psychology services and over eighty percent of coaches said they had some fundamental sport psychology education or experience. Despite these results, baseball coaches reported being unsure if their peers are using sport psychology services, and may not be discussing their sport psychology experiences with each other.

It is likely that a value added service like sport psychology would go through the normal progression of public consumption before being a widely accepted component of teams across competition levels. For example, a select group of coaches might initially work with SPPs, and as they talked more openly with other coaches about the value of working with an SPP and how implementing sport psychology principles during training impacted performance, other coaches would be encouraged to hire an SPPs as well. As this snowball process continued, one would assume the sport psychology 'movement' would progress somewhat consistently. Despite coaches' apparent willingness to talk about their work with SPPs, it remains unclear how or when those conversations are taking place. SPPs seem to be unclear about how coaches are communicating their sport psychology experience with other coaches, and perhaps more importantly, the steps coaches are willing to take to overcome the barriers (e.g., time and money) that may prohibit them from hiring an SPP. Therefore, it may be beneficial for SPPs to learn about the types of conversations coaches are having with one another about their work with SPPs, and ask questions about how they obtain, understand, and apply sport psychology principles with their athletes.

\section{Limitations of the Current Study}

There are several perceived limitations in the current study. First, the timing of study may have had a negative effect on participation rates because most teams were just beginning 


\section{AN EXPLORATORY INVESTIGATION OF BASEBALL COACHES’ ATTITUDES}

their 2013 season during the initial contact and data collection period. Therefore, coaches who may have been inclined to complete the survey in the off-season would not be as likely to participate in the current study. Despite the rather large sample size, it is important to note that nearly $75 \%$ of the coaches contacted did not participate in the survey. Over 6,000 emails went unanswered, so the results of the study may only be generalized to the baseball coaches who completed the survey. There is a possibility that only those coaches interested in sport psychology responded to the initial email request. Therefore, the remainder of ABCA members and NCAA Head Coaches who did not participate may have responded differently to the survey.

Second, self-selection bias is another limitation of the current study (Wittmer et al., 1999). In any given population, some people may be more likely than others to open an email request for participation and/or take an online survey. Similarly, the coaches who viewed sport psychology in a positive light may have been more likely to respond to the email request and participate in the study. It is possible that coaches who had negative experience or attitudes towards sport psychology decided not to participate, resulting in a final sample of coaches who were genuinely interested in sport psychology and had positive sport psychology experiences.

Third, neither the SPA-RC nor SPA-RC 2 had been widely used in previous research. To date, there are only two published studies using the instrument (Zakrajsek \& Zizzi, 2007; Zakrajsek, et al., 2011). Although the SPA-RC has limited previous use, the psychometric properties have been examined revealing the four subscales are psychometrically sound. However, the survey in the current study had additional items that have not been psychometrically tested. Therefore, only descriptive statistical analysis could be completed on non-SPA-RC items. Also, coaches were asked about their intention to use sport psychology services within the next 12 months "with adequate financial resources". Coaches may not have 


\section{AN EXPLORATORY INVESTIGATION OF BASEBALL COACHES’ ATTITUDES}

the financial support to hire an SPP or use sport psychology services, so their responses may have reflected their "ideal" situation rather than their actual intentions. More research should be done to further examine the psychometric properties of the modified instrument. This is also the first study examining only baseball coaches, therefore, the findings of the study may not be generalized to any other sport coaches.

Fourth, the questionnaire was administered through a host website and all scores were self-reported, so there is no way to be confident that the intended participant was actually the one who completed the survey. Since all data was self-reported, so also there was no way to ensure coaches' responses represented their actual attitudes and experiences with sport psychology. The researcher was not able to control for the condition under which each survey was completed. Participants completing the SPA-RC in previous research have done so under the direct supervision head researchers; in the current study, participants completed the survey at their own convenience. While this method may have increased the total sample used for analysis, conditions (e.g., home vs. office, computer vs. cell phone, alone vs. with others) for completing the instrument likely varied.

Finally, social desirability may have impacted coaches' responded to each survey item. The overwhelming majority of coaches said they intended to us sport psychology services within the next 12 months with adequate financial resources. On average, coaches reported that they had generally positive attitudes towards sport psychology, that they valued the expertise that SPPs can offer their teams, and that they believed over $65 \%$ of baseball performance could be attributed to psychological factors. However, only $33 \%$ of coaches said they were currently using some form of sport psychology services. The discrepancy between coaches' self-reported scores and the percentage of coaches currently using sport psychology services may suggest that 


\section{AN EXPLORATORY INVESTIGATION OF BASEBALL COACHES' ATTITUDES}

coaches inflated their responses based on what they believed other coaches think about sport psychology.

\section{Recommendations for Future Research}

Several recommendations for future research seem useful. Collecting data in late February and early March may have eliminated some coaches from the potential sample. Coaches are very busy at that time of year since baseball seasons start in early spring. Further research with baseball coaches should be done in the mid to late fall, or at times where baseball is not "in season". Future research should also clearly define the primary competition level coached (i.e., youth, high school, college, professional) by each participant. It is likely that coaches place more or less emphasis on the psychological aspects of performance based on the age and skill level of their athletes. A clear understanding of how the level of importance coaches place on the on psychological skills at each competition level could help SPPs target and market their services more effectively.

Future studies on this topic should clearly define the type of services baseball coaches prefer and intend to use. Coaches from the current study reported a wide range of sport psychology experiences (e.g., books, workshops, team and individual services), so they may have an expectation or preference for a specific method of services delivery. For example, some coaches may be more likely to prefer an SPP give a 1-2 hour workshop on a specific PST topic than hire him or her on staff for the entire season. Therefore, more information on coaches' preferred method of sport psychology service would help SPPs meet the mental training needs of baseball coaches.

Coaches generally reported a high interest level and intention to use sport psychology services. However, the survey item used to measure "intention" was phrased in a way that 


\section{AN EXPLORATORY INVESTIGATION OF BASEBALL COACHES’ ATTITUDES}

coaches' could answer without worrying about the financial implications of such a decision. Therefore, future research should examine coaches' intention to use sport psychology services taking into account their current financial resources (See Appendix B). Research should also address the additional barriers that may prohibit or limit coaches from utilizing sport psychology services. A follow up survey could also be sent to each respondent 12 months after the initial contact to determine if they used SP services within the past year

Future research investigating baseball coaches' relationship with sport psychology could include a pre-post test experimental design with a workshop or seminar as the 'treatment'. Regarding the results that suggest coaches are confident in their ability to deliver sport psychology services, it would be interesting to determine if coaches' scores were consistent before and after a sport psychology intervention. Coaches may not know what they do not know; in other words, they may be confident in doing sport psychology themselves, but would not know if they were doing it 'right' or 'wrong'. Perhaps only after an intervention would coaches be alerted to the level of sport and exercise science training and education, communication skills, and content delivery methods necessary to be a competent and qualified sport psychology professional. Future research examining coaches' intentions to use sport psychology services should take into consideration their current financial resources instead of providing them with what may be unrealistic circumstances (i.e., adequate financial resources). Since coaches reported strong intentions to use sport psychology services replacing the words “adequate financial resources" with "current financial resources" may reveal more accurate intentions.

To date, research investigating coaches' attitudes toward sport psychology has been limited, both in the number of sports examined and the demographic characteristics of coaches 


\section{AN EXPLORATORY INVESTIGATION OF BASEBALL COACHES’ ATTITUDES}

who have completed the SPA-RC. Therefore, further studies utilizing the SPA-RC should be done across sports and competition levels. A larger sample size and examining coaches from female-dominated sports (e.g. gymnastics, softball, women's lacrosse, women's soccer, women's golf, etc.) could provide more responses from female and minority coaches with regard to factors such as age, years experience, skill level coached, stigma tolerance, confidence in SP consultation, openness to sport psychology, and cultural preference for SPPs. Finally, future research should be conducted with baseball coaches to replicate the results of the current study, and researchers should utilize the SPA-RC with more coaches from a broad range of team and individual sports.

\section{Conclusion}

In conclusion, the purpose of the current study was to explore baseball coaches' attitudes and experiences with sport psychology. The results of this unique study are encouraging; it appears that baseball coaches who responded to the survey generally reported positive attitudes towards sport psychology, valued the expertise and skills SPPs could provide their teams, had had positive experiences with sport psychology services, and intended to use sport psychology services in the future. Although coaches appeared to be generally confident in their ability to implement and apply sport psychology principles with their teams, it remains unclear how baseball coaches are applying SP with their athletes and if their techniques are successful. Also, there is a discrepancy between the level of importance coaches' placed on the psychological aspects of performance and the amount of time coaches' said they dedicated to sport psychology training. The current study failed to explore the barriers prohibiting coaches from spending more time developing their athletes' mental skills. 


\section{AN EXPLORATORY INVESTIGATION OF BASEBALL COACHES’ ATTITUDES}

The implications of these results are important to baseball coaches, athletes, athletic administrators, and sport psychology professionals. Coaches reported positive attitudes and subjective norms towards sport psychology, and appeared to value and appreciate the skills and services that SPPs can provide their teams. Applying the results of the study to the Theory of Reasoned Action, it appears the majority of respondents were primed to utilize sport psychology service sometime in the next 12 months. However, results suggested coaches did not know that their peers thought positively about sport psychology or that other coaches were open to the idea of using sport psychology services. To bridge the gap, SPPs could be proactive and discuss results of the current study with organizational decision-makers and baseball coaches. Knowing that one out of every three coaches are using SP services in some form or another, and approximately $90 \%$ of coaches would implement SP with their athletes and teams if they had the financial resources might encourage some coaches to utilize sport psychology services.

Over 1,500 baseball coaches thought a survey on the psychological aspect of performance was important enough to dedicate time and effort to completing. Over 500 coaches were interested in participating in future research on the topic. Results of the regression analysis suggested sport psychology experience increases likelihood of engaging in sport psychology consultation. This information should challenge SPPs to think of new and creative ways of exposing baseball coaches and athletes to sport psychology education experiences such as workshops and podcasts. SPPs should be encouraged to know that there is a market across baseball to impart the knowledge and training they have received on coaches and players; it is up to SPPs to work with coaches to come up with ways for sport psychology to be integrated throughout the baseball community. 


\section{AN EXPLORATORY INVESTIGATION OF BASEBALL COACHES’ ATTITUDES}

The current study investigated baseball coaches' attitudes and experiences with sport psychology. With a better understanding of these constructs, SPPs may be more informed about how to educate, instruct, and implement sport psychology services with baseball teams and coaches. With a clearer understanding of baseball coaches' attitudes and experiences with sport psychology, SPPs may develop more effective methods of teaching baseball players and coaches the mental skills that could help improve individual and team performance. This information could also improve baseball coaches' understanding of sport psychology concepts, helping to bridge the gap between research, theory, and practice. 
AN EXPLORATORY INVESTIGATION OF BASEBALL COACHES’ ATTITUDES

\section{References}

Adie, J. W., \& Jowett, S. (2010). Meta-perceptions of the coach-athlete relationship, achievement goals, and intrinsic motivation among sport participants. Journal of Applied Social Psychology, 40(11), 2750-2773.

Ajzen, I. (1991). The theory of planned behavior. Organizational Behavior and Human Decision Processes, 50, 197-211.

Ajzen, I., \& Fishbein, M. (1980). Predictions of goal directed behavior: Attitudes, intention, and perceived behavioral control. Journal of Experimental Social Psychology, 22, 453-474.

American College Baseball Coaches Association Directory. (2012). Retrieved from http:// www.abca.org.

Anderson, A. G., Hodge, K. P., Lavallee, D., and Martin, S. B. (2004). New Zealand athletes' attitudes towards seeking sport psychology consultation. New Zealand Journal of Psychology, (33)3, 129-136.

Anderson, A. A., Miles, A. A., Robinson, P. P., \& Mahoney, C. C. (2004). Evaluating the athlete's perception of the sport psychologist's effectiveness: What should we be assessing? Psychology Of Sport \& Exercise, 5(3), 255-277.

Anshel, M. (1990). Sport psychology: From theory to practice. Scottsdale, AZ. Gorsuch Scarisbrick Publishers.

Atkinson, D. R., \& Lowe, S. M. (1995). The role of ethnicity, cultural knowledge, and conventional techniques in counseling and psychotherapy. In J. G. Ponterotto, J. M. Casas, L. A. Suzuki, \& C. M. Alexander (Eds.), Handbook of multicultural counseling (pp. 387414). Thousand Oaks, CA: Sage.

Benjamin, L. (1986). Why don't they understand us: A history of psychology's public image. American Psychologist, 41(9), 941-946. 
AN EXPLORATORY INVESTIGATION OF BASEBALL COACHES' ATTITUDES

Berg, B. L. (2007) Qualitative research methods for the social sciences ( $6^{\text {th }}$ ed.). Boston, MA: Allyn \& Bacon

Berger, J. M., Levant, R., McMillan, K., Kelleher, W., \& Sellers, A. (2005). Impact of gender role conflict, traditional masculinity ideology, alexithymia, and age on men's attitudes toward psychological help seeking. Psychology of Men \& Masculinity, 6(1), 73-78.

Blom, L. C., Hardy, C. J., Burke, K. L., \& Joyner, A. (2003). High school athletes' perceptions of sport psychology and preferences for services. International Sports Journal, 7(2), 18.

Brooks, J. E., \& Bull, S. J. (1999). Perceptions of the sport psychologist by female university athletes. Journal Of Sports Sciences, 17(3), 205-212.

Buckley, G. I., \& Malouff, J. M. (2005). Using modeling and vicarious reinforcement to produce more positive attitudes towards mental health treatment. The Journal of Psychology, 139, 197-209.

Campbell, D. T., \& Stanley, J. C. (1963). Experimental and quasi-experimental designs for research on teaching. In N. L. Gage (Ed.), Handbook of research on teaching (pp. 171-246). Chicago, IL: Rand McNally.

Cohen, J. (1988). Statistical power analysis for the behavioral sciences $\left(2^{\text {nd }}\right.$ ed.) Hillsdale, NJ: Lawrence Erbaum.

Constantine, M., \& Gainor, K. (2004). Depressive symptoms and attitudes toward counseling as predictors of biracial college women's psychological help-seeking behavior. Women \& Therapy, 27(1/2), 147-158.

Côté, J., Salmela, J. H, \& Russell, S. (1995). The knowledge of high-performance gymnastic coaches: Methodological framework. The Sport Psychologist, 9, 65-75. 
AN EXPLORATORY INVESTIGATION OF BASEBALL COACHES' ATTITUDES

Cratty, B. J. (1973). Psychology in contemporary sport: Guidelines for coaches and athletes, Prentice-Hall, Englewood Cliffs, N.J.

Creswell, J. W. (2009). Research design: Qualitative, quantitative, and mixed methods approaches (3rd ed.). Thousand Oaks, CA US: Sage Publications, Inc.

Denzin, N. K. (1978). The Research act: A theoretical introduction to sociological methods $\left(2^{\text {nd }}\right.$ ed.). New York: McGraw Hill.

Dieffenbach, K., Gould, D., Moffett, A. (2002). The coach's role in developing champions. International Journal of Volleyball Research, 5, 30-32.

Dillman, D. A. (2007). Mail and Internet surveys: The tailored design method (2 $2^{\text {nd }}$ ed.). Hoboken, NJ US: John Wiley \& Sons Inc.

Donohue, B., Covassin, T., Lancer, K., Dickens, Y., Miller, Y., Hash, A., et al. (2004). Examination of psychiatric symptoms in student-athletes. Journal of General Psychology, 131, 29-35.

Dunn, J. H., \& Holt, N. L. (2003). Collegiate ice hockey players' perceptions of the delivery of an applied sport psychology program. The Sport Psychologist, 17(3), 351.

English Dictionary. (2012). Retrieved from http://dictionary.reference.com.

Esters, I., Cooker, P., \& Ittenbach, R. (1998). Effects of a unit of instruction in mental health on rural adolescents' conceptions of mental illness and attitudes about seeking help. Adolescence, 33(130), 469.

Etzel, E. F., Watson, J., \& Zizzi, S. (2004). A web-based survey of AAASP members' ethical beliefs and behaviors in the new millennium. Journal of Applied Sport Psychology, 16 (3), 236-250. 
Ferraro, T. T., \& Rush, S. S. (2000). Why athletes resist sport psychology. Athletic Insight, 2(3). Retrieved from November 9, 2012, from www.athleticinsight.com.

Field, A. (2009). Discovering statistics using SPSS ( $3^{\text {rd }}$ Ed). Thousand Oaks, CA: Sage

Figone, A. J. (1999). When the physical breaks down, try a little A.S.P. Coach \& Athletic Director, 68(9), 4.

Fischer, E. H., \& Turner, J. L. (1970). Orientations to seeking professional help: development and research utility of an attitude scale. Journal of Consulting And Clinical Psychology, 35(1), 79-90.

Fishbein, M., \& Ajzen, I. (2010). Predicting and changing behavior: The reasoned action approach. New York, NY US: Psychology Press.

Frey, M., Laguna, P. L., \& Ravizza, K. (2003). Collegiate athletes' mental skill use and perceptions of success: An exploration of the practice and competition settings. Journal of Applied Sport Psychology, 15, 115-128.

Futterman, M. (2011). As baseball season begins, kids are losing interest in the national pastime. In Wall Street Journal. Retrieved November 19, 2012, from http://online.wsj.com/article/SB10001424052748703712504576232753156582750.html

Garland, A. F., \& Zigler, E. F. (1994). Psychosocial correlates of help-seeking attitudes among children and adolescents. American Journal of Orthopsychiatry, 64(4), 586.

Giacobbi, P. R., Roper, E., Whitney, J., Butryn, T. (2002). College coaches' views about the development of successful athletes: A descriptive exploratory investigation. Journal of Sport Behavior, 25(2), 166-180.

Gmelch, G. G. (2000). Baseball's mental game. Nine: A Journal of Baseball History \& Culture, $9(1 / 2), 208-224$. 
Gmelch, G. (2006). Inside pitch: Life in professional baseball. University of Nebraska Press. Lincoln, NE.

Good, G. K. (1995). Male gender role conflict, depression, and help seeking: Do college men face double jeopardy? Journal of Counseling \& Development, 74(1), 70.

Godin, G. G., \& Shephard, R. J. (1990). Use of attitude-behavior models in exercise promotion. Sports Medicine, 10(2), 103-121.

Gould, D., Chung, Y., Smith, P., \& White, J. (2006). Future directions in coaching life skills: Understanding high school coaches' views and needs. Athletic Insight: The Online Journal of Sport Psychology, 8(3), 1-9.

Gould, D. D., Dieffenbach, K. K., \& Moffett, A. A. (2002). Psychological characteristics and their development in Olympic champions. Journal of Applied Sport Psychology, 14(3), $172-204$.

Gould, D., Hodge, K., Peterson, K., \& Petlichkoff, L. (1987). Psychological foundations of coaching: Similarities and differences among intercollegiate wrestling coaches. The Sport Psychologist, 1(4), 293-308.

Gould, D. D., Medbery, R. R., Damarjian, N. N., \& Lauer, L. L. (1999). A survey of mental Skills training knowledge, opinions, and practices of junior tennis coaches. Journal of Applied Sport Psychology, 11(1), 28-50.

Gould, D., Murphy, S., Tammen, V., \& May, J. (1991). An evaluation of U.S. Olympic sport psychology consultant effectiveness. The Sport Psychologist, 5(2), 111-127.

Gould, D., Petlichkoff, L., Hodge, K., \& Simons, J. (1990). Evaluating the effectiveness of a psychological skills educational workshop. The Sport Psychologist, 4(3), 249-260.

Gratton, C., \& Jones, I. (2004). Research methods for sport studies. New York: Routledge. 
AN EXPLORATORY INVESTIGATION OF BASEBALL COACHES' ATTITUDES

Haberl, P., \& Peterson, K. (2006). Olympic-size ethical dilemmas: Issues and challenges for sport psychology consultants on the road and at the Olympic games. Ethics \& Behavior, $16(1), 25-40$.

Hall, C. R., \& Rodgers, W. M. (1989). Enhancing coaching effectiveness in figure skating through a mental skills training program. The Sport Psychologist, 3(2), 142-154.

Hanson, T. \& Ravizza, K. (1998). Heads-up baseball: Playing the game one pitch at a time. Chicago, IL. Masters Press.

Hanson, T., \& Ravizza, K. (2003). Issues for the sport psychology professional in baseball. The Psychology of Team Sports, 1,191-215.

Harris, D. V., \& Harris, B. L. (1984). Athlete's guide to sports psychology: mental skills for physical people. New York; United States: Leisure Press.

Harrell, F. E. (2001). Regression modeling strategies: With applications to linear models, logistic regression, and survival analysis. New York: Springer Verlag.

Huck, S., Cormier, W., \& Bounds, W. G. (1974). Reading Statistics and Research. New York: Harper \& Row Publishers.

Janssen, J. (1994). Championship team-building. Tucson, AZ. Winning the Mental Game.

Kornspan, A. S., \& MacCracken, M. J. (2003). The use of psychology in professional baseball: The pioneering work of David F. Tracy. Nine: A Journal of Baseball History and Culture, 11, 36-43.

Kozar, B., \& Mechikoff, R. A. (1983). Sport psychology: The coach's perspective. Ipswich, MA. Thomas, C.C.

Lambert, P. (2007). Client perspectives on counseling: Before, during and after. Counseling \& Psychotherapy Research, 7(2), 106-113.

Lane, J. M., \& Addis, M. E. (2005). Male gender role conflict and patterns of help seeking in 
AN EXPLORATORY INVESTIGATION OF BASEBALL COACHES’ ATTITUDES

Costa Rica and the United States. Psychology of Men \& Masculinity, 6(3), 155-168.

Lapchick, R.E., \& Harrison, K. (2011). The 2011 racial and gender report card: Major League Baseball. www.tidesport.org

LaRose, B. (1988). What can the sport psychology consultant learn from the educational consultant? The Sport Psychologist, 2(2), 141-153.

LeUnes, A. (2000). Coleman Robert Griffith: American psychologist. In A.E. Kazdin (Ed.), Encyclopedia of psychology. Washington, D.C.: American Psychological Association.

Lubker, J. R., Visek, A. J., Geer, J. R., \& Watson II, J. C. (2008). Characteristics of an effective sport psychology consultant: Perspectives from athletes and consultants. Journal of Sport Behavior, 31(2), 147-165.

Lubker, J. R., Watson, II, J. C., Visek, A. J., \& Geer, J. R. (2005). Physical appearance and the perceived effectiveness of performance enhancement consultants. The Sport Psychologist, 19(4), 446.

Ludwig, M. M. (1996). A sport psychology perspective. The Journal of Physical Education, Recreation \& Dance, 67(5), 31.

Magnusen, M. J., \& Rhea, D. J. (2009). Division 1 athletes' attitudes towards and preferences for male and female strength and conditioning coaches. Journal of Strength \& Conditioning Research (Lippincott Williams \& Wilkins), 23(4), 1084-1090.

Maher, C. (2011). The complete mental game of baseball: Taking charge of the process, on and off the field. USA: Authorhouse.

Martens, R. (1987). Coaches guide to sport psychology. Champaign, IL: Human Kinetics Martin, F., \& Lumsden, J. (1987). Coaching: An effective behavioral Approach. St Louis: Times Mirror/Mosby. 
AN EXPLORATORY INVESTIGATION OF BASEBALL COACHES' ATTITUDES

Martin, S. B. (2005). High school and college athletes' attitudes toward sport psychology. Journal of Applied Sport Psychology, 17(2), 127-139.

Martin, S. B., Kellmann, M. M., Lavallee, D. D., \& Page, S. J. (2002). Development and psychometric evaluation of the sport psychology attitudes - revised form: A multiple group investigation. The Sport Psychologist, 16(3), 272-290.

Martin, S. B., Wrisberg, C. A., Beitel, P. A., \& Lounsbury, J. J. (1997). NCCA Division I athletes' attitudes toward seeking sport psychology consultation: The development of an objective instrument. The Sport Psychologist, 11(2), 201-218.

Martin, S. B., Zakrajsek, R. A., \& Wrisberg, C. A. (2012). Attitudes towards sport psychology and seeking assistance: Key factors and a proposed model. In C. Logan \& M. Hodge (Eds.) Psychology of Attitudes (pp. 1-35). Hauppauge, NY: Nova Science Publishers, Inc.

Masuda, A., Suzumura, K., Beauchamp, K. L., Howells, G. N., \& Clay, C. (2005). United States and Japanese college students' attitudes toward seeking professional psychological help. International Journal of Psychology, 40(5), 303-313.

Monserrat, J. M. (2004). The effect of self-administered VMBR on batting performance among collegiate baseball players. Dissertation Abstracts International, 65, 2104.

Morrison, M., De Man, A .F., \& Drumheller, A. (1993). Correlates of socially restrictive and authoritarian attitudes towards mental patients in university students. Social Behavior and Personality, 21, 333-338.

National Collegiate Athletic Association Baseball Coaches Listing. (2013). Retrieved from http://web1.ncaa.org/stats/StatsSrv/careersearch.

Neelis, L. A. (2008). NCAA Division I head softball coaches confidence, openness, and stigma tolerance towards sport psychology consultants. Unpublished Doctoral Dissertation. 
AN EXPLORATORY INVESTIGATION OF BASEBALL COACHES’ ATTITUDES

O'Loughlin, R., Duberstein, P., Veazie, P., Bell, R., Rochlen, A. E, \& Kravitz, R. (2011). Role of the gender-linked norm of toughness in the decision to engage in treatment for depression. Psychiatric Services, 62(7), 740-746.

Orlick, T., \& Partington, J. (1987). The sport psychology consultant: Analysis of critical components as viewed by Canadian Olympic athletes. The Sport Psychologist, 1(1), 4-17.

Pain, M., \& Harwood, C. (2004). Knowledge and perceptions of sport psychology within English soccer. Journal of Sports Sciences, 22(9), 813-826.

Parslow, R. A., \& Jorm, A. F. (2000). Who uses mental health services in Australia? An analysis of data from the National Survey of Mental Health and Wellbeing. Australian And New Zealand Journal of Psychiatry, 34(6), 997-1008.

Partington, J., \& Orlick, T. (1987). The sport psychology consultant: Olympic coaches' views. The Sport Psychologist, 1(2), 95-102.

Patton, M. (2002). Qualitative research and evaluation methods. Thousand Oaks, CA: Sage Publications.

Raskin, J. D. (2002). Constructivism in psychology: Personal construct psychology, radical constructivism, and social constructivism. In J. D. Raskin \& S. K. Bridges (Eds.), Studies in meaning: Exploring constructivist psychology (pp. 1-25). New York, NY: Pace University Press.

Ravizza, K. (1988). Gaining entry with athletic personnel for season-long consulting. The Sport Psychologist, 2(3), 243-254.

Ravizza, K. (1990). Sportpsych consultation issues in professional baseball. The Sport Psychologist, 4(4), 330-340.

Rice, T. (1997). Should coaches administer psychological interventions to their athletes? 
AN EXPLORATORY INVESTIGATION OF BASEBALL COACHES' ATTITUDES

Dissertation Abstracts International Section A, 57.

Rubin, H. J., \& Rubin, I. S. (1995). Qualitative interviewing: The art of hearing data. London: Sage.

Schinke, R. J., Hancock, D., Dubuc, N. G., \& Dorsch, K. D. (2006). Looking to the future of sport psychology: An introduction. Athletic Insight: The Online Journal of Sport Psychology, 8(3), 1-9.

Silva, J. M. (1984). The status of sport psychology: A national survey of coaches. Journal of Physical Education, Recreation, and Dance, 55, 46-49.

Speed, H. D., Andersen, M. B., \& Simons, J. (2005). The selling or the telling of sport psychology; presenting services to coaches. In M. B. Andersen (Ed.), Sport psychology in practice (pp. 1-16). Champaign, IL: Human Kinetics.

Sullivan, J., \& Hodge, K. P. (1991). A survey of coaches and athletes about sport psychology in New Zealand. The Sport Psychologist, 5(2), 140-151.

Thelwell, R., Greenless, I. A., \& Weston, N. V. (2005). The impact of psychological skills training on football performance. (Abstract). Journal of Sports Sciences, 23(2), 160-161.

Tinsley, H. F. A. (1982). Expectations about counseling. Unpublished test manual. Carbondale, IL. Southern Illinois University, Department of Psychology.

Tod, D. (2007). The long and winding road: Professional development in sport psychology. The Sport Psychologist, 21(1), 94-108.

Tracy, D. (1951). The psychologist at bat. New York: Sterling.

Trafimow, D., \& Miller, A. (1996). Predicting and understanding mental practice. Journal of Social Psychology, 136(2), 173-180.

Tutko, T. A., \& Richards, J. W. (1971). Psychology of coaching. Boston; United States: Allyn \& 
AN EXPLORATORY INVESTIGATION OF BASEBALL COACHES’ ATTITUDES

Bacon.

Voight, M., \& Callaghan, J. (2001). The Use of Sport Psychology Services at NCAA Division I Universities From 1998-1999. The Sport Psychologist, 15(1), 91.

Walker, E.E., \& Eslinger, O.W. (2003). Sport psychology consulting: Coaches’ attitudes toward seeking sport psychology consultation: The development of an objective measure.

Wardinsky, R., \& LeUnes, A. (2006). Coleman Griffith: Reflections of a baseball visionary. Applied Research in Coaching and Athletics Annual, 1-8.

Watson, J. C. (2006). Student-athletes and counseling: Factors influencing the decision to seek counseling services. College Student Journal, 40(1), 35-42.

Watson, J., Zizzi, S., \& Etzel, E. F. (2006). Ethical training in sport psychology programs: Current training standards. Ethics \& Behavior, 16(1), 5-14.

Weinberg, R. S., \& Gould, D. D. (2003). Introduction to psychological skills training. In Weinberg, R.S. (ed.), Foundations of sport \& exercise psychology. ( $3^{\text {rd }}$ ed., pp. 241-262) Champaign, IL: Human Kinetics

Weinberg, R. S., \& Gould, D. (2011). Foundations of sport and exercise psychology (5th ed.). Champaign, IL US: Human Kinetics.

Werthner, P., \& Trudel, P. (2006). A new theoretical perspective for understanding how coaches learn to coach. The Sport Psychologist, 20(2), 198-212.

Wesner, M. L., \& Valiance, J. (2007). Athletes' preference for gender of team physician. Clinical Journal Of Sport Medicine, 17(2), 143-144.

Williams, J. M., \& Straub, W. F. (2006). Sport psychology: Past, present, future. In, J. M. Williams (Ed.), Applied sport psychology: personal growth to peak performance $\left(5^{\text {th }}\right.$ ed., 
pp. 1-14). Boston: McGraw Hill.

Wittmer, D. F., Colman, R. W., \& Katzman, S. L. (1999). From paper-and-pencil to screenand-keyboard: Toward a methodology for survey research on the Internet. In S. Jones (Ed.), Doing Internet Research: Critical Issues and Methods for Examining the Net (pp. 145-161). Thousand Oaks, CA: Sage.

Wrisberg, C. A., Loberg, L. A., Simpson, D., Withycombe, J. L., \& Reed, A. (2010). An exploratory investigation of NCAA division-I coaches' support of sport psychology consultants and willingness to seek mental training services. The Sport Psychologist, 24 (4), 489-503.

Wrisberg, C. A., \& Marin, S. B. (1994). Attitudes of African-American and Caucasian athletes towards sport psychology consultants. Ninth annual conference of the Association of the Advancement of Applied Sport Psychology. Lake Tahoe, NV.

Wrisberg, C. A., Simpson, D., Loberg, L. A., Withycombe, J. L., \& Reed, A. (2009). NCAA Division-I student-athletes' receptivity to mental skills training by sport psychology consultants. The Sport Psychologist, 23(4), 470-486.

Wrisberg, C., Withycombe, J., Simpson, D., Loberg, L. A., \& Reed, A. (2012). NCAA Division-I administrators' perceptions of the benefits of sport psychology services and possible roles for a consultant. The Sport Psychologist, 26(1), 16-28.

Zakrajsek, R. A., Martin, S. B., \& Zizzi, S. J. (2011). American high School football coaches' attitudes toward sport psychology consultation and intentions to use sport psychology services. International Journal of Sports Science \& Coaching, 6(3), 461-478.

Zakrajsek, R. A., \& Zizzi, S. J. (2007). Factors influencing track and swimming coaches’ 
intentions to use sport psychology services. Athletic Insight: The Online Journal of Sport Psychology, 9(2), Retrieved February 14, 2011, from http://www.athleticinsight.com/Vol9Iss2/CoachesIntentions.htm.

Zakrajsek, R. A., \& Zizzi, S. J. (2008). How do coaches' attitudes change when exposed to a sport psychology workshop? Journal of Coaching Education, 1(1). 


\section{Tables}

Table 1

\section{Demographic Characteristics of Participants}

Table 1

Demographic Characteristics of Participants $(N=1,401)$

\begin{tabular}{|c|c|c|}
\hline & Percent (\%) & $N$ \\
\hline \multicolumn{3}{|l|}{ Gender } \\
\hline Male & 99.6 & 1385 \\
\hline Female & 0.36 & 5 \\
\hline \multicolumn{3}{|l|}{ Familiar with Sport Psychology } \\
\hline Yes & 88.31 & 1231 \\
\hline No & 11.69 & 163 \\
\hline \multicolumn{3}{|l|}{ Age Group } \\
\hline $18-25$ & 6.81 & 94 \\
\hline $26-35$ & 28.82 & 398 \\
\hline $36-45$ & 25.63 & 354 \\
\hline $45-55$ & 21.07 & 291 \\
\hline $56-65$ & 12.53 & 173 \\
\hline $66+$ & 5.14 & 71 \\
\hline \multicolumn{3}{|l|}{ Race } \\
\hline African American & 1.39 & 19 \\
\hline American Indian & .37 & 5 \\
\hline Asian & .88 & 12 \\
\hline Pacific Islander & .37 & 5 \\
\hline Caucasian & 91.52 & 1252 \\
\hline Hispanic Another Spanish Origin & 5.48 & 75 \\
\hline \multicolumn{3}{|l|}{ Education Level } \\
\hline High School & 4.82 & 66 \\
\hline Bachelor's Degree & 40.39 & 553 \\
\hline Master's Degree & 51.35 & 703 \\
\hline Doctorate Level Degree & 3.43 & 47 \\
\hline \multicolumn{3}{|l|}{ Athlete Level Coached } \\
\hline Youth or High School & 66.17 & 927 \\
\hline Collegiate & 49.96 & 700 \\
\hline Professional or Olympic & 7.28 & 102 \\
\hline \multicolumn{3}{|c|}{ Previous Sport Psychology Experience } \\
\hline Yes & 42.97 & 602 \\
\hline No & 57.03 & 799 \\
\hline \multicolumn{3}{|c|}{ Intentions to Use Sport Psychology Services } \\
\hline Yes & 89.54 & 1164 \\
\hline No & 10.46 & 136 \\
\hline
\end{tabular}

Values based on number of valid responses per item 
AN EXPLORATORY INVESTIGATION OF BASEBALL COACHES’ ATTITUDES

Table 2

Primary Job Responsibility by "Baseball Mental Game" Demographic Variables

Table 2

Primary Job Responsibility by "Baseball Mental Game” Demographic Variables

\begin{tabular}{|c|c|c|c|c|c|}
\hline $\begin{array}{l}\text { Primary Job of } \\
\text { Participant }\end{array}$ & $\begin{array}{r}\text { Age } \\
(\bar{x})\end{array}$ & $\begin{array}{c}\text { Years } \\
\text { Coaching } \\
(\bar{x})\end{array}$ & $\begin{array}{c}\text { \% of } \\
\text { Baseball } \\
\text { "Mental" } \\
(\bar{x})\end{array}$ & $\begin{array}{l}\text { \% Practice time } \\
\text { on "Mental } \\
\text { Game" } \\
(\bar{x})\end{array}$ & $\begin{array}{l}\text { \% Current or } \\
\text { Previous SP Use } \\
(\bar{x})\end{array}$ \\
\hline $\begin{array}{l}\text { Head Coach } \\
(n=761)\end{array}$ & 44.2 & 20.14 & $71.45 \%$ & $32.07 \%$ & $44.9 \%$ \\
\hline $\begin{array}{l}\text { Associate Coach } \\
(n=75)\end{array}$ & 39.84 & 15.17 & $72.53 \%$ & $26.05 \%$ & $45.3 \%$ \\
\hline $\begin{array}{l}\text { Assistant Coach } \\
(n=314)\end{array}$ & 37.05 & 13.50 & $70.31 \%$ & $23.22 \%$ & $35.7 \%$ \\
\hline $\begin{array}{l}\text { Position Coach } \\
(n=82)\end{array}$ & 38.67 & 14.86 & $68.79 \%$ & $20.01 \%$ & $45.1 \%$ \\
\hline $\begin{array}{l}\text { Volunteer Coach } \\
(n=61)\end{array}$ & 43.18 & 14.74 & $69.03 \%$ & $15.10 \%$ & $32.8 \%$ \\
\hline $\begin{array}{l}\text { Private Instructor } \\
(n=37)\end{array}$ & 45.41 & 19.26 & $69.34 \%$ & $23.09 \%$ & $51.4 \%$ \\
\hline $\begin{array}{l}\text { Strength Coach } \\
(\mathrm{n}=3)\end{array}$ & 41.67 & 17.33 & $75.00 \%$ & $23.33 \%$ & $66.7 \%$ \\
\hline $\begin{array}{l}\text { Administrator } \\
(n=26)\end{array}$ & 53.42 & 28.83 & $60.80 \%$ & $21.74 \%$ & $57.7 \%$ \\
\hline Total $(n=1359)$ & 42.55 & 18.08 & $68.19 \%$ & $23.91 \%$ & $43.0 \%$ \\
\hline
\end{tabular}

*. Values based on number of valid responses per item 
Table 3a

Demographic Variables and Attitudes Towards Sport Psychology

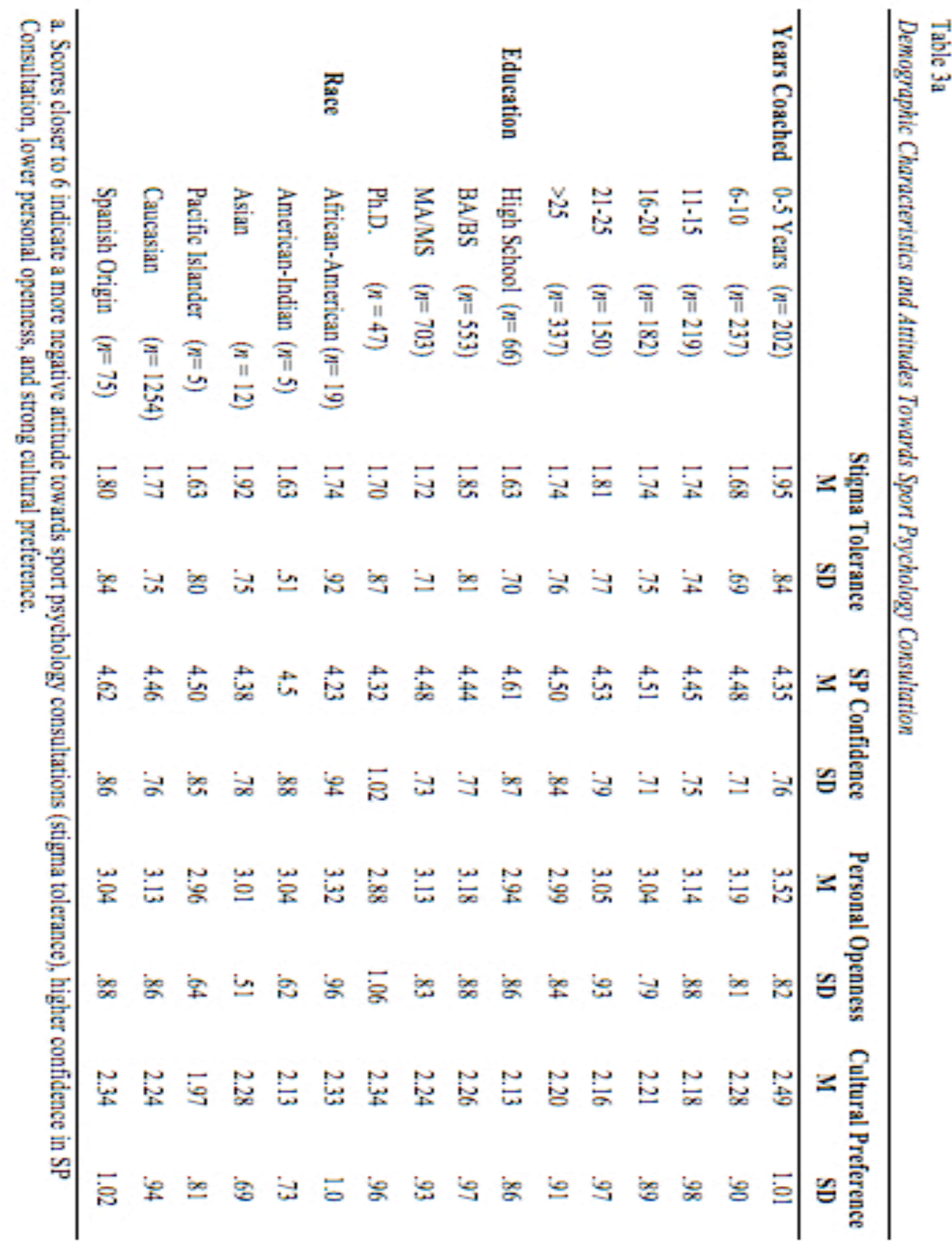


Table 3b

Demographic Variables and Attitudes Towards Sport Psychology

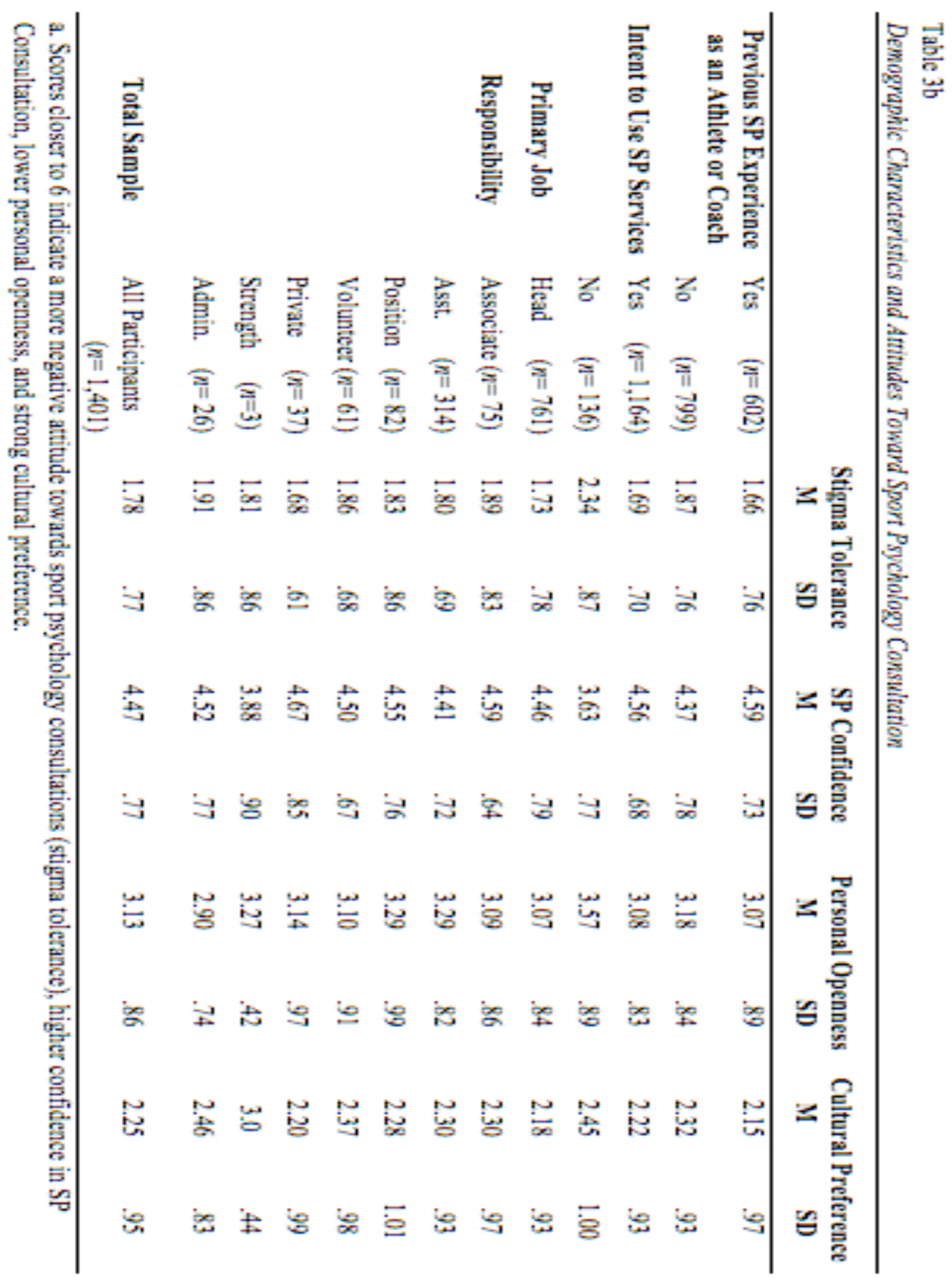


Table 4

\section{Descriptive Statistics and Correlations for Non-SPARC-2 Items}

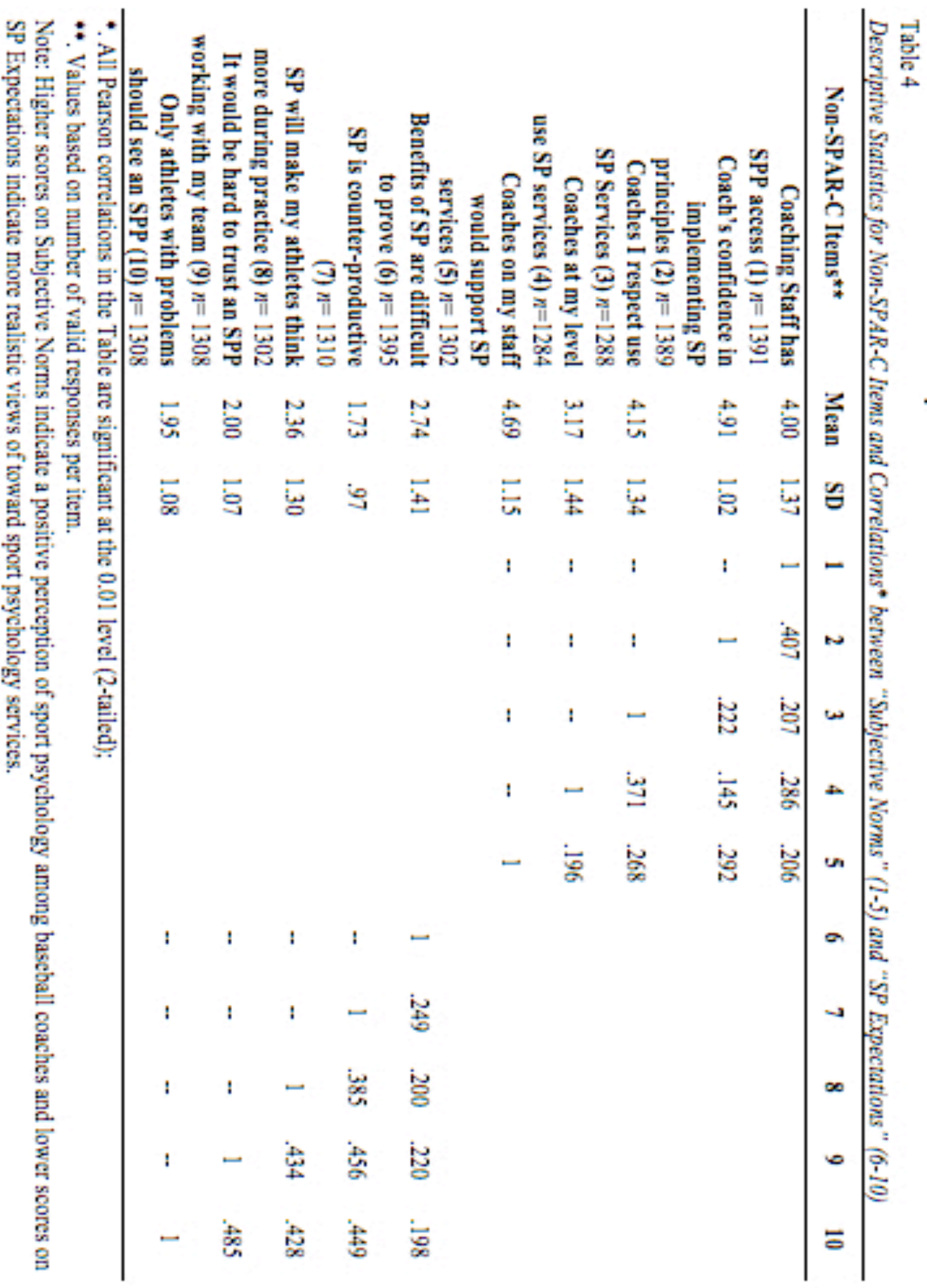




\section{Table 5a}

Independent Samples t-Test for "Intent to Use SP Services"
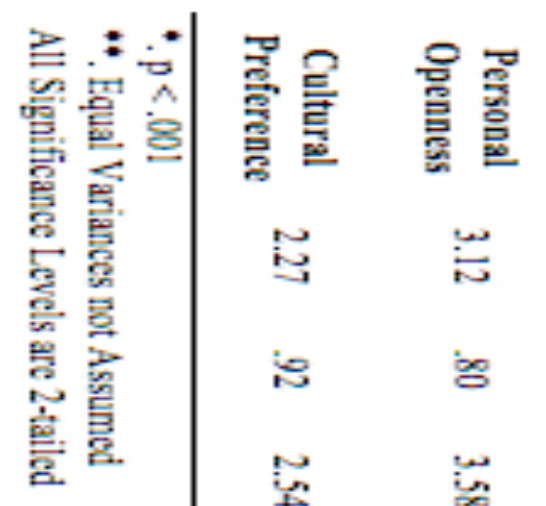

$\infty$

is

旁量总高

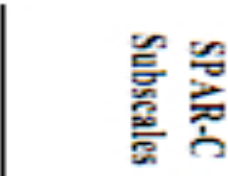

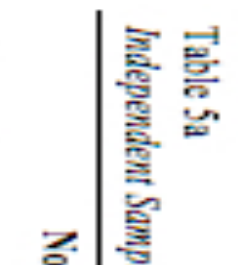

료을

is

is

岕

岕

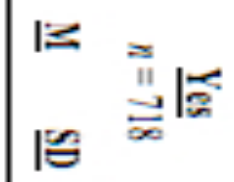

g

莺

is

$\infty$

\$

응

安

夏

$\stackrel{2}{\alpha}$

s

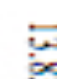

$\infty$

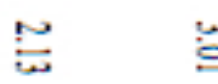

is

$\infty$

$\infty \quad$ 文

$\Xi \quad \dot{0} \quad \dot{0}$

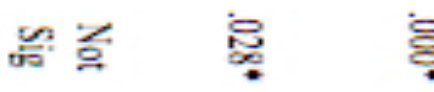

$\ddot{q}$

乌

§ु

웅

I:

$\because \underset{3}{=}$

들

$\stackrel{7}{=}$ 
Table 5b

Independent Samples t-Test for "Intent to Use SP Services"

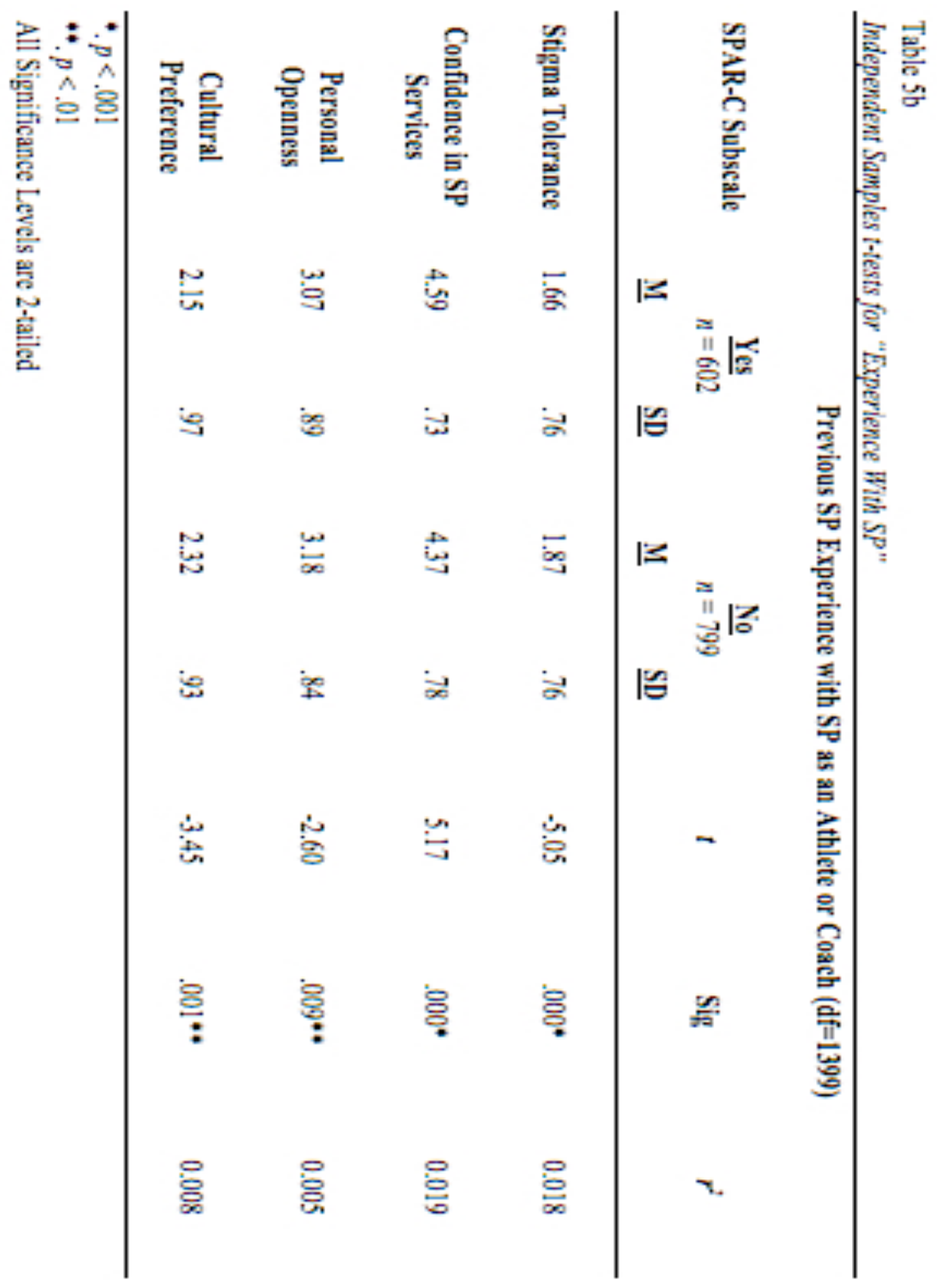


Table 6

Correlations for SPA-RC Subscales

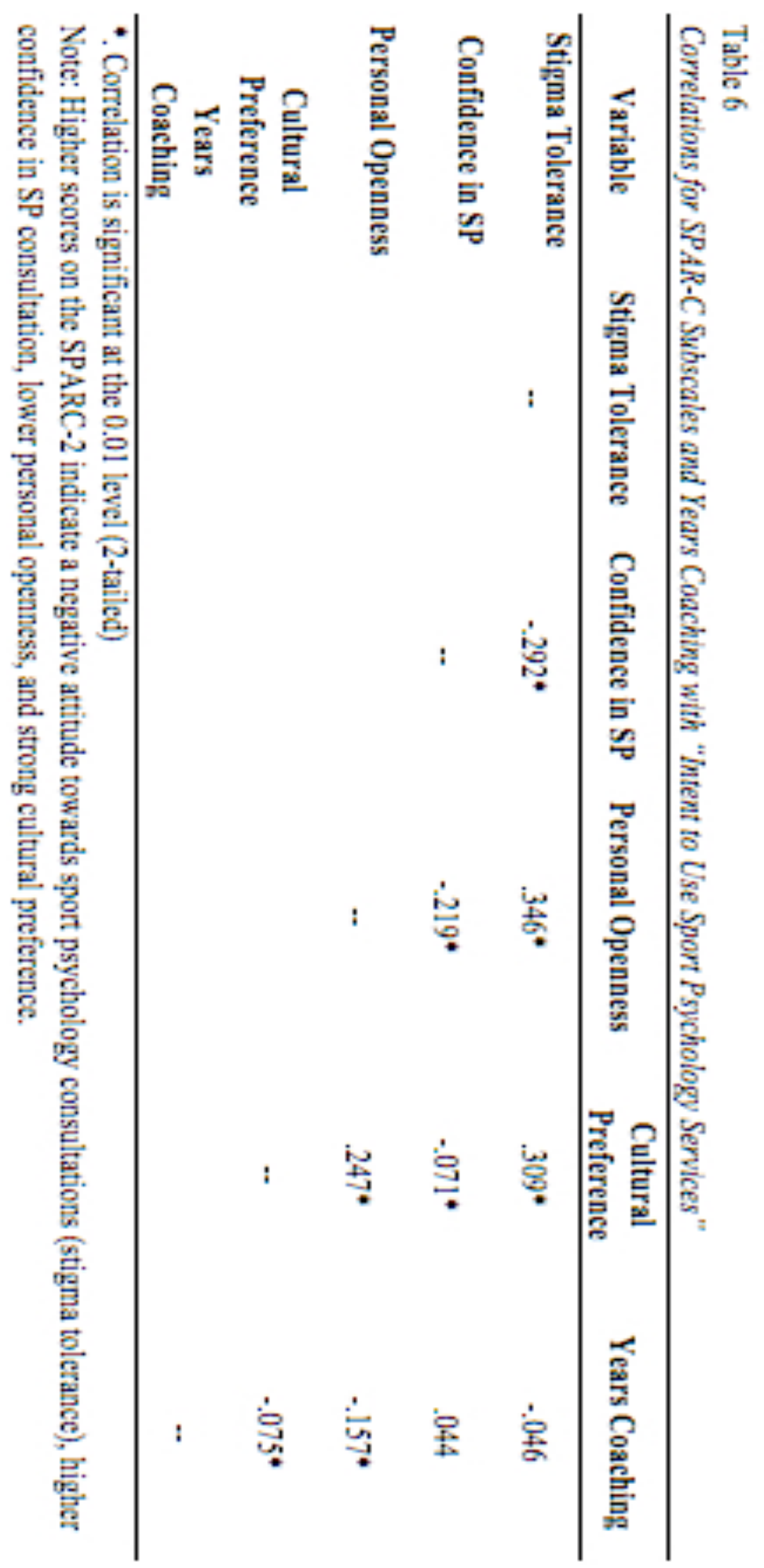




\section{Table 7}

Table 7

\section{Intent to Use SP Services in the Next 12 Months}

Intent to Use SP Services in Next 12 Months by Independent Variable

\begin{tabular}{|c|c|c|c|c|}
\hline Intent to Use SP Services & $\begin{array}{l}\text { No (\%) } \\
n=136\end{array}$ & $\begin{array}{l}\text { Yes }(\%) \\
n=1164 \\
\end{array}$ & $\begin{array}{c}\text { Total } \\
N=1,300\end{array}$ & $p$-value ${ }^{a}$ \\
\hline Previous Sport Psychology & & & & $.000^{*}$ \\
\hline \multicolumn{5}{|l|}{ Experience as a Player or Coach } \\
\hline No & 15.1 & 84.9 & 713 & \\
\hline Yes & 4.8 & 95.2 & 587 & \\
\hline Years Coaching & & & & .078 \\
\hline $0-5$ & 13.8 & 86.2 & 247 & \\
\hline $6-11$ & 14.6 & 85.4 & 233 & \\
\hline $11-15$ & 11 & 89 & 210 & \\
\hline $16-20$ & 7 & 93 & 173 & \\
\hline $21-25$ & 14.4 & 85.6 & 139 & \\
\hline $26+$ & 7.7 & 92.3 & 298 & \\
\hline Education Level & & & & .078 \\
\hline High School/Bachelor's Degree & 12.1 & 87.9 & 572 & \\
\hline Master's/Doctoral Degree & 9 & 91 & 708 & \\
\hline \multicolumn{5}{|l|}{ SPARC-2 Subscales } \\
\hline Stigma Tolerance & & & & $.000 *$ \\
\hline Quartile 1 & 3.8 & 96.2 & 499 & \\
\hline Quartile 2 & 10.8 & 89.2 & 158 & \\
\hline Quartile 3 & 9.3 & 90.7 & 375 & \\
\hline Quartile 4 & 24.3 & 75.7 & 268 & \\
\hline Confidence in SP Services & & & & $.000^{*}$ \\
\hline Quartile 1 & 26.5 & 73.5 & 355 & \\
\hline Quartile 2 & 8.0 & 92.0 & 323 & \\
\hline Quartile 3 & 4.0 & 96.0 & 328 & \\
\hline Quartile 4 & 1.0 & 99.0 & 294 & \\
\hline Personal Openness & & & & $.000^{*}$ \\
\hline Quartile 1 & 6.4 & 93.6 & 392 & \\
\hline Quartile 2 & 5.3 & 94.7 & 320 & \\
\hline Quartile 3 & 12.7 & 87.3 & 355 & \\
\hline Quartile 4 & 21.0 & 79.0 & 233 & \\
\hline Cultural Preference & & & & .091 \\
\hline Quartile 1 & 8.3 & 91.7 & 399 & \\
\hline Quartile 2 & 10.3 & 89.7 & 310 & \\
\hline Quartile 3 & 10.1 & 89.9 & 327 & \\
\hline Quartile 4 & 14.4 & 85.6 & 264 & \\
\hline
\end{tabular}

${ }^{\mathrm{a}}$. Pearson Chi-Square Test Statistic

$*$. $\mathrm{p}<.001$ 
AN EXPLORATORY INVESTIGATION OF BASEBALL COACHES’ATTITUDES

Table 8

\section{Logistic Regression and Odds Ratios For Intention to Use SP Service}

Table 8

Odds Ratios (95\% Confidence Intervals) from logistic regression for participants who "Intend to use SP services in the next 12 months"

\begin{tabular}{|c|c|c|c|}
\hline & $\begin{array}{c}\text { Adjusted } \\
\text { Odds Ratio }\end{array}$ & $95 \% \mathrm{CI}$ & $p$-value \\
\hline \multicolumn{4}{|c|}{ Previous Sport Psychology Experience as a Player or } \\
\hline No & 1.00 & & \\
\hline Yes & 2.91 & $1.81,4.70$ & $.000 *$ \\
\hline \multicolumn{4}{|l|}{ Years Coaching } \\
\hline $0-5$ & 1.00 & & \\
\hline $6-11$ & 1.11 & $0.58,2.11$ & .752 \\
\hline $11-15$ & 1.07 & $0.55,2.06$ & .848 \\
\hline $16-20$ & 1.69 & $0.78,3.64$ & .189 \\
\hline $21-25$ & 0.59 & $0.29,1.21$ & .151 \\
\hline $26+$ & 1.26 & $0.66,2.41$ & .478 \\
\hline \multicolumn{4}{|l|}{ Education Level } \\
\hline High School/Bachelor's Degree & 1.00 & & \\
\hline Master's/Doctoral Degree & 1.16 & $0.77,1.76$ & .484 \\
\hline \multicolumn{4}{|l|}{ SPARC-2 Subscales ${ }^{a}$} \\
\hline \multicolumn{4}{|l|}{ Stigma Tolerance } \\
\hline Quartile 1 (0.00-1.43) & 1.00 & & \\
\hline Quartile 2 (1.43-1.57) & 0.37 & $0.18,0.81$ & $.012 *$ \\
\hline Quartile 3 (1.57-2.29) & 0.66 & $0.35,1.23$ & .191 \\
\hline Quartile 4 (2.29-6.00) & 0.30 & $0.16,0.56$ & $.000 *$ \\
\hline \multicolumn{4}{|l|}{ Confidence in SP Services } \\
\hline Quartile 1 (0.00-3.99) & 1.00 & & $.000 *$ \\
\hline Quartile 2 (4.00-4.49) & 3.68 & $2.24,6.05$ & $.000 *$ \\
\hline Quartile 3 (4.50-4.99) & 6.20 & $3.28,11.73$ & $.000 *$ \\
\hline Quartile 4 (5.00-6.00) & 36.65 & 8.72 .154 .09 & $.000 *$ \\
\hline \multicolumn{4}{|l|}{ Personal Openness } \\
\hline Quartile 1 (0.00-2.59) & 1.00 & & \\
\hline Quartile $2(2.60-3.19)$ & 2.07 & $1.03,4.15$ & $.041 *$ \\
\hline Quartile $3(3.20-379)$ & 1.14 & $0.63,2.06$ & .660 \\
\hline Quartile 4 (3.80-6.00) & 0.60 & $0.33,1.11$ & .102 \\
\hline \multicolumn{4}{|l|}{ Cultural Preference } \\
\hline Quartile 1 (0.00-1.49) & 1.00 & & \\
\hline Quartile $2(1.50-2.15)$ & 0.97 & $0.55,1.74$ & .926 \\
\hline Quartile 3 (2.16-2.99) & 1.50 & $0.83,2.70$ & .178 \\
\hline Quartile 4 (3.00-6.00) & 1.05 & $0.58,1.90$ & .880 \\
\hline
\end{tabular}

CI: Confidence Interval, ${ }^{\mathrm{a}}$ Quartile Scores on each SPARC-2 Subscale; **. $\mathrm{p}<.001$

Note: Higher scores on the SPARC-2 indicate a negative attitude towards sport psychology consultations (stigma tolerance), higher confidence in SP consultation, lower personal openness, and strong cultural preference. 


\title{
Appendices
}

\section{Appendix A}

\section{Sport Psychology Attitudes Revised Coaches-2}

\author{
Sport Psychology Attitudes Revised Coaches-2 (SPARC-2)
}

PART 2: Please indicate your level of agreement with each of the following statements by circling the response that corresponds to your feelings toward each statement. Please respond to each statement as truthfully as you can

\begin{tabular}{|c|c|c|c|c|c|}
\hline SD & MD & D & A & MA & SA \\
\hline 1 & 2 & 3 & 4 & 5 & 6 \\
\hline Strongly & Moderately & Silghtly & Silghtly & Moderately & Strongly \\
\hline Disagree & Dlsagree & Disagree & Agree & Agree & Agree \\
\hline
\end{tabular}

Question (SPC- Sport Psychology Consultant)

1. A sport psychology consutant (SPC) can help athletes Improve their mental toughness.

2. If an athlete on my team asked my advice about personal feellngs of fallure related to sport, I would recommend that helshe see a SPC.

3. I would not want a SPC working with my athletes because other coaches would think less of me.

4. A good Idea for an athlete to avold personal worrles and concerns is to keep one's mind on the job.

5. I would like to have the assistance of a SPC to help me better understand my athletes.

6. I would feel uneasy having a SPC work with my athletes because some people would disapprove.

7. There is something respectable in the attitude of athletes who are willing to cope with their conflicts and fears without resorting to professional help.

8. If I utillized a SPC to help me coach better, I would not want other coaches to know about it.

9. I feel that an athlete with emotional problems during sport performance would feel most secure in recelving assistance from a SPC.

10. Having seen a SPC is bad for an athlete's reputation.

11. If I was worrled or upset about my athletes' performance, I would want to get help from a SPC.

12. If I were to hire a SPC, I would take into account his/her race or ethnicity.

13. Athletes emotional dimcultles tend to work themselves out in time.

14. I think a SPC would help my athletes perform better under pressure.

15. I would not want someone else to know about my athletes recelving help from a SPC.

\begin{tabular}{|c|c|c|c|c|c|}
\hline SD & MD & D & A & MA & SA \\
\hline 1 & 2 & 3 & 4 & 5 & 6 \\
\hline 1 & 2 & 3 & 4 & 5 & 6 \\
\hline 1 & 2 & 3 & 4 & 5 & 6 \\
\hline 1 & 2 & 3 & 4 & 5 & 6 \\
\hline 1 & 2 & 3 & 4 & 5 & 6 \\
\hline 1 & 2 & 3 & 4 & 5 & 6 \\
\hline 1 & 2 & 3 & 4 & 5 & 6 \\
\hline 1 & 2 & 3 & 4 & 5 & 6 \\
\hline 1 & 2 & 3 & 4 & 5 & 6 \\
\hline 1 & 2 & 3 & 4 & 5 & 6 \\
\hline 1 & 2 & 3 & 4 & 5 & 6 \\
\hline 1 & 2 & 3 & 4 & 5 & 6 \\
\hline 1 & 2 & 3 & 4 & 5 & 6 \\
\hline 1 & 2 & 3 & 4 & 5 & 6 \\
\hline 1 & 2 & 3 & 4 & 5 & 6 \\
\hline
\end{tabular}


AN EXPLORATORY INVESTIGATION OF BASEBALL COACHES' ATTITUDES

\section{Appendix A (cont'd)}

\section{Sport Psychology Attitudes Revised Coaches-2}

(SPC= Sport Psychology Consultant)

16. There are great differences between people of different races or ethnicitles.

17. A SPC could help my athletes fine-tune thelr performance.

18. If my athletes worked with a SPC, I would not want other coaches to know about it.

19. My athletes would be more comfortable with a SPC if heishe were of the same race or ethnicity as them.

20. At times I have felt lost and would have welcomed professional advice for a personal problem.

21. I would think less of my athletes if they went to a SPC.

22. Athletes with a strong character can get over mental confilcts by themselves.

23. I would be more comfortable with a SPC If he/she were the same race or ethnicity as I am.

24. An athlete may relate best to a SPC If heishe were the same race or ethniclty.

25. Athletes should know how to handle problems without needing assistance from a SPC.

26. I would be more comfortable hiring a SPC If helshe were from the same cultural background as my athletes.
SD MD D A MA SA

$\begin{array}{lllllll}1 & 2 & 3 & 4 & 5 & 6 & \mathrm{CP}\end{array}$

$\begin{array}{lllllll}1 & 2 & 3 & 4 & 5 & 6 & 60\end{array}$

$\begin{array}{lllllll}1 & 2 & 3 & 4 & 5 & 6 & \text { 5ा }\end{array}$

$\begin{array}{lllllll}1 & 2 & 3 & 4 & 5 & 6 & \mathrm{CP}\end{array}$

$\begin{array}{lllllll}1 & 2 & 3 & 4 & 5 & 6 & \text { co }\end{array}$

$\begin{array}{lllllll}1 & 2 & 3 & 4 & 5 & 6 & \text { का }\end{array}$

$\begin{array}{lllllll}1 & 2 & 3 & 4 & 5 & 6 & \text { PO }\end{array}$

$\begin{array}{lllllll}1 & 2 & 3 & 4 & 5 & 6 & c P\end{array}$

$\begin{array}{lllllll}1 & 2 & 3 & 4 & 5 & 6 & C P\end{array}$

$\begin{array}{lllllll}1 & 2 & 3 & 4 & 5 & 6 & \text { PO }\end{array}$

$\begin{array}{lllllll}1 & 2 & 3 & 4 & 5 & 6 & \text { CP }\end{array}$ 
AN EXPLORATORY INVESTIGATION OF BASEBALL COACHES’ ATTITUDES

\section{Appendix B}

\section{Sport Psychology Access and Experience}

\section{Sport Psychology Access and Experience}

Please indicate your level of agreement with each of the following statements and questions by marking the response that corresponds to your feelings toward each statement. Please respond to each statement as truthfully as you can.

\begin{tabular}{|c|c|c|}
\hline $\begin{array}{l}\text { Does your cosching staff currently have acsess to } \\
\text { sport psychology services? }\end{array}$ & Yes & No \\
\hline $\begin{array}{l}\text { Do your athletes currently have access to sport } \\
\text { psychology services? }\end{array}$ & Yes & No \\
\hline $\begin{array}{l}\text { Does your coaching staff currently use sport } \\
\text { psychology services? }\end{array}$ & Yes & No \\
\hline $\begin{array}{l}\text { Do your athletes currently use sport psychology } \\
\text { services? }\end{array}$ & Yes & No \\
\hline $\begin{array}{l}\text { If no, has your coaching staff or athlates previously } \\
\text { used sport psychology services? }\end{array}$ & Yes & No \\
\hline $\begin{array}{l}\text { If yes, are/were you satisfied with the sport } \\
\text { psychology services you received? }\end{array}$ & Yes & No \\
\hline \multicolumn{3}{|c|}{ Please describe why you do, or do not, use sport psycbology services: } \\
\hline $\begin{array}{l}\text { If you had proper financial resources, would you } \\
\text { use sport psychology services in the next calendar } \\
\text { year? }\end{array}$ & Yes & No \\
\hline
\end{tabular}


AN EXPLORATORY INVESTIGATION OF BASEBALL COACHES' ATTITUDES

\title{
Appendix C
}

\section{Demographic Questions}

\author{
Please answer the following questions by marking the most appropriate response: \\ Gender \\ Age \\ Year you began coaching baseball \\ State or Territory \\ Race or Ethnicity: \\ Highest Level of Education \\ Primary Job Title: \\ Youth \\ High School \\ College \\ Division \\ Professional \\ Head Coach \\ Assistant Coach \\ Volunteer Coach \\ Level(s) of baseball players you currently coach or work with (mark all that apply) \\ Youth \\ Trave1/Select/Club \\ High School \\ Adult/Recreational \\ Junior College \\ NALA \\ NCAA Division 1 \\ NCAA Division II \\ NCAA Division III \\ International/Olympic \\ Professional
}


AN EXPLORATORY INVESTIGATION OF BASEBALL COACHES' ATTITUDES

\section{Appendix D}

\section{Sport Psychology Expectations and Baseball Norms Questionnaire}

\section{Sport Psychology Expectations and Baseball Norms Questionnaire}

Please indicate your level of agreement with each of the following statements and cuestions by marking the response that corresponds to your feelings toward each statement. Please

$+$ respond to each statement as truthfully as you can.

\begin{tabular}{|c|c|c|c|c|c|}
\hline $\begin{array}{c}\text { SD } \\
1 \\
\text { Strongly } \\
\text { Dissgroe }\end{array}$ & $\begin{array}{c}\text { MD } \\
2 \\
\text { Moderately } \\
\text { Disagree }\end{array}$ & $\begin{array}{c}\text { D } \\
3 \\
\text { Slightly } \\
\text { Disagree }\end{array}$ & $\begin{array}{c}\text { A } \\
4 \\
\text { Slightly } \\
\text { Agree }\end{array}$ & $\begin{array}{c}\text { MA } \\
5 \\
\text { Maderately } \\
\text { Agree }\end{array}$ & $\begin{array}{c}\text { SA } \\
6 \\
\text { Strongly } \\
\text { Agree }\end{array}$ \\
\hline
\end{tabular}

\begin{tabular}{|l|c|c|}
\hline $\begin{array}{l}\text { 1. I am familiar with sport psychology and the range } \\
\text { of services sport psychology professionals provide. }\end{array}$ & Yes & No \\
\hline
\end{tabular}

If Yes, please move on to $\mathbf{H} 2$.

If No, please read the following description:

Sport psychology looks at the psychological factors that influence performance and determine strategies to help maximize performance during competition.

Sport psychology professionals help athletes and coaches manage the challenges athletes face during competition.

Sport psychology services can include, but are not limited to: Mental Skills Training, Teamsuilding, Imagery, Relaxation, Leadership Confidence, Focus, Stress Management, Goal-Setting, Time Management, Improving the Cosch-Athlete Relationship, and other Performance Enhancement Strategies

\begin{tabular}{|l|l|c|c|c|c|c|c|}
\hline $\begin{array}{l}\text { Please use the scale above (where appropriate) to } \\
\text { answer the following questions by marking the } \\
\text { most appropriate response. }\end{array}$ & SD & MD & D & A & MA & SA \\
\hline $\begin{array}{l}\text { 1. It is important for my athletes to have strong } \\
\text { mental skills. }\end{array}$ & 1 & 2 & 3 & 4 & 5 & 6 \\
\hline $\begin{array}{l}\text { 2. If the coaching staff wanted to, we could easily } \\
\text { access and use sport psychology services. }\end{array}$ & 1 & 2 & 3 & 4 & 5 & 6 \\
\hline $\begin{array}{l}\text { 3. The benefits of sport psychology services are } \\
\text { difficult to prove. }\end{array}$ & 1 & 2 & 3 & 4 & 5 & 6 \\
\hline $\begin{array}{l}\text { 4. I am confident implementing sport psychology } \\
\text { principles with my team. }\end{array}$ & 1 & 2 & 3 & 4 & 5 & 6 \\
\hline
\end{tabular}


AN EXPLORATORY INVESTIGATION OF BASEBALL COACHES' ATTITUDES

\section{Appendix D (cont'd)}

\section{Sport Psychology Expectations and Baseball Norms Questionnaire}

\section{Sport Psychology Expectations and Baseball Norms Questionnaire}

Please indicate your level of agreement with each of the following statements and cuestions by marking the response that corresponds to your feelings toward each statement. Please respond to each statement as truthfully as you can.

\begin{tabular}{|c|c|c|c|c|c|c|c|c|}
\hline $\begin{array}{c}\text { SD } \\
1 \\
\text { Strongly } \\
\text { Dissgree }\end{array}$ & $\begin{array}{c}\text { MD } \\
2 \\
\text { Moderately } \\
\text { Disagree }\end{array}$ & $\begin{array}{c}\text { D } \\
3 \\
\text { Slightly } \\
\text { Disagree }\end{array}$ & $\begin{array}{c}\text { A } \\
4 \\
\text { Slightly } \\
\text { Agree }\end{array}$ & & $\begin{array}{r}\text { M } \\
5 \\
\text { lader } \\
\text { Agr }\end{array}$ & & $\begin{array}{r}\mathrm{S} \\
\text { Stro } \\
\mathrm{Ag}\end{array}$ & \\
\hline \multicolumn{3}{|c|}{$\begin{array}{l}\text { Please use the scale above (where appropriate) to } \\
\text { answer the following questions by marking the } \\
\text { most appropriate response. }\end{array}$} & SD & MD & D & $\boldsymbol{A}$ & MA & SA \\
\hline \multicolumn{3}{|c|}{$\begin{array}{l}\text { The cosches whose opinions I respect use sport } \\
\text { psychology services with their athletes and teams. }\end{array}$} & 1 & 2 & 3 & 4 & 5 & 6 \\
\hline \multicolumn{3}{|c|}{$\begin{array}{l}\text { Using sport psychology services would be } \\
\text { sounter-productive; athletes either have mental } \\
\text { skills or they don't. }\end{array}$} & 1 & 2 & 3 & 4 & 5 & 6 \\
\hline \multicolumn{3}{|c|}{$\begin{array}{l}\text { Other coaches at my level are using sport } \\
\text { psychology services with their athletes. }\end{array}$} & 1 & 2 & 3 & 4 & 5 & 6 \\
\hline \multicolumn{3}{|c|}{$\begin{array}{l}\text { Using sport psychology services might make our } \\
\text { athletes' think too much during practice. }\end{array}$} & 1 & 2 & 3 & 4 & 5 & 6 \\
\hline \multicolumn{3}{|c|}{$\begin{array}{l}\text { Other coaches on our staff would be supportive of } \\
\text { using sport psychology services with our athletes. }\end{array}$} & 1 & 2 & 3 & 4 & 5 & 6 \\
\hline \multicolumn{3}{|c|}{$\begin{array}{l}\text { It would be difficult for me to trust a sport } \\
\text { psychology consultant working with our athletes. }\end{array}$} & 1 & 2 & 3 & 4 & 5 & 6 \\
\hline \multicolumn{3}{|c|}{$\begin{array}{l}\text { The only appropriate time to seek out sport } \\
\text { psychology services is when there is a problem. }\end{array}$} & 1 & 2 & 3 & 4 & 5 & 6 \\
\hline
\end{tabular}




\section{Appendix E}

\section{Sport Psychology Education and Baseball-Related Questions}

Please list any coaching-related certifications you have below:

Please indicate your previous educational experiences with sport psychology (mark all that apply)

Taken at least one course in general psychology

Taken at least one course in sport psychology

Read at least one book on sport psychology

Attended at least one workshop on sport psychology

During your athletic career, did you or any of your teams ever use sport psychology services or work with a sport psychology professional?

Yes

No

Not Applicable

In your opinion, what percent of baseball is mental?

\%

What percentage of time with your athletes is devoted to mental training?

$\%$

How and/or when do you integrate sport psychology or mental skills with your team? (Please provide specific examples)

In your opinion, what is the most important aspect of the "Mental Game" and how could a Sport Psychology Consultant help you or your athletes improve that area of performance?

Please describe why you use/do not use sport psychology services: 


\section{Appendix F}

\section{WVU Institutional Review Board Approval}

IRB protocol number: 1212008624

Title: An Exploratory Investigation of Baseball Coaches Attitudes, Knowledge, and Experiences With Sport Psychology

\section{PI: Edward Etzel}

The West Virginia University Institutional Review Board approved the above-referenced protocol on $\{$ PROTOCOL_LAST_APPROVAL_DATE\}. To access this protocol, click on the protocol number link provided. Your approval letter can be found in the History subsection of the Summary \& History section located on the Protocol Actions page. For more information, see the Viewing Correspondence quick reference guide. Any future protocol action requests can be completed through the WVU+kc system.

Questions related to NHSR, Full Board, Emergency Use, Clinical Trials, or CIRB protocols as well as amendments, renewals, deviations/violations/exceptions, or adverse events/UPIRTSOs should be directed to Lilo Ast at 304.293.7555 or lilo.ast@mail.wvu.edu.

Questions related to Exempt or Expedited protocols should be directed to Barbara White at 304.293.5971 or barb.white@mail.wvu.edu.

Training or troubleshooting questions should be directed to Jonathan Young at 304.293.1119 or jonathan.young@mail.wvu.edu. 


\section{Appendix G}

\section{Pre-Notice Letter}

\section{Hi [FirstName],}

As Hall of Fame catcher and Yankee legend Yogi Berra famously said, "Baseball is 90\% mental, the other half is physical". While that may be up for debate, we want to know what you think!

We have invited every American Baseball Coaches Association (ABCA) Member and NCAA Head Baseball Coach to participate in the first nationwide research project on sport psychology and the "Mental Game" of baseball. For participating, you will receive free coaching resources about the "Mental Game", mental training, and additional information about sport psychology.

[FirstName], you have a tremendous impact on your players' athletic and personal development. Based on the influence you have on the players and coaches around you, your thoughts and opinions are extremely important. Your participation can help influence how players and coaches learn about this critical aspect of the game.

On Wednesday February 13th, you will receive an email with more information about the project. The email will include a personalized link to a brief 10-15 minute survey about baseball and sport psychology.

If you would like to take the survey now, please click here:

[SurveyLink]

Participation is completely voluntary and anonymous. The information collected will be used to better meet the "Mental Game" needs of players in coaches across the country. Results will be used to provide coaches and players with better resources about the "Mental Game", and help improve how sport psychology professionals communicate this information to players and baseball professionals.

Thank you in advance for participating and your continued dedication to the game of baseball.

Jesse Michel, M.S.

Doctoral Candidate - Sport Psychology

West Virginia University

Edward Etzel, Ed. D.

College of Physical Activity and Sport Sciences

P.O. Box 6116, Morgantown, WV 26506-6116

West Virginia University's Institutional Review Board (IRB) has acknowledgment of this study on file.

[RemoveLink] 


\section{Appendix H}

\section{Cover Letter of Explanation}

This is a follow up to last week's email about the first nationwide research project on sport psychology and the "Mental Game" of baseball. Over 8,500 baseball coaches across the country have been invited to participate, and the information collected will be used to provide ABCA Members, coaches, and players of all age groups and skill levels with helpful resources and information related to this critical area of the game.

For participating, you will receive free coaching resources about the "Mental Game", mental training, and additional information about sport psychology.

[FirstName], please click the personalized link below to participate and be taken to a secure website to complete the 10-15 minute survey. [SurveyLink]

A final reminder email will be sent on Tuesday February 26th, and the survey will be available until Friday March 1st at 11:59PM PST. To opt out of future emails, please click the link at the bottom of this letter.

Thank you in advance for your time and continued dedication to the game of baseball.

Jesse Michel, M.S.

Doctoral Candidate - Sport and Exercise Psychology

West Virginia University

Edward Etzel, Ed. D.

College of Physical Activity and Sport Sciences

P.O. Box 6116, Morgantown, WV 26506-6116

West Virginia University's Institutional Review Board (IRB) has acknowledgment of this study on file.

Click to opt-out of future emails: [RemoveLink] 


\section{Appendix I}

\section{Consent Form}

Dear Participant,

You are invited to take part in a nationwide research project about sport psychology and baseball. The study is being conducted by Jesse Michel, M.S., in partial requirement for a doctoral degree in Sport and Exercise Psychology at West Virginia University. The project is being supervised by Dr. Edward Etzel, Professor and Licensed Psychologist in the College of Physical Activity and Sport Sciences.

The purpose of this project is to understand your attitudes towards the "mental" game of baseball, and learn about your sport psychology knowledge and experiences. Learning what you think about this component of performance will help mental training experts and sport psychology consultants provide better services to coaches, athletes, and baseball professionals across age groups and skill levels. After completing the survey, you will have access to a link with free sport psychology information and will receive additional coaching resources about "the mental game". You may also request a copy of the results from the study and provide your contact information if you want to be included in further research on this topic. Your participation will take approximately 10 minutes and is completely voluntary.

You will not be asked to provide any information that may identify you as a participant and you must be 18 years of age or older to be eligible for the study. There are no known risks or costs for participating, and your involvement in this project will be kept anonymous and as confidential as legally possible. All data will be collected and reported as a group, so no individual responses will be identified. You can skip any question you do not wish to answer and may discontinue at any time. West Virginia University's Institutional Review Board (IRB) has acknowledgment of this study on file.

On behalf of West Virginia University, we thank you in advance for your time, continued dedication to the game of baseball, and willingness to participate in the study. Should you have any questions about this form or the research project, please feel free to contact Jesse Michel at (818) 632-4017 or by e-mail at Jesse.michel@mail.wvu.edu.

By clicking "Next" at the bottom of the page, you agree to the above terms and to participate in the project.

Good luck this season!

Jesse Michel, M.S., M.A.

Doctoral Candidate - Sport and Exercise Psychology

Edward Etzel, Ed. D.

College of Physical Activity and Sport Sciences

West Virginia University

P.O. Box 6116

Morgantown, WV 26506-6116 